\title{
Development of a Novel Pressure-Sensitive Mat Using Proximity Sensors
}

by

Roger Cristiano Degasperi Selzler

A thesis submitted to the Faculty of Graduate Studies and Research in partial fulfillment of the requirements for the degree of

Masters of Applied Science

in

\section{Electrical and Computer Engineering with Specialization in Data Science}

Carleton University

Ottawa, Ontario

(C)2018

Roger Cristiano Degasperi Selzler 


\section{Abstract}

Pressure sensitive mats (PSM) have been widely used in research for measuring contact pressure. In the field of non-obtrusive patient monitoring, PSM have been used in a number of applications including finding the center of pressure for patients in different positions, monitoring heart and breathing rates of a patient laying on a bed, and monitoring and controlling the amount of time in which a patient remains in the same position, thus avoiding pressure ulcers. However, the cost of such devices remains high, and the deployment of PSMs in actual clinical practice is rare. This thesis presents the design of a new pressure sensitive mat technology using infrared proximity sensors and evaluates its performance relative to the state of the art.

A flexible printed circuit board with sixteen solid-state infrared proximity sensors is prototyped and interfaced with a development LaunchPad microcontroller board. Graphical user interfaces are developed in MATLAB and as a native Microsoft Windows application to acquire data from the prototype board. A silicone rubber layer comprising multiple cavities is designed and manufactured, such that applied pressure reduces the distance from the cavity's reflective surface to the proximity sensor, thereby transducing pressure into displacement, into optical intensity, and into an electrical signal. The linearity and metrological properties of the implemented prototype are evaluated and compared with existing technologies, where improved performance was observed. The prototype was positioned beneath a subject's back while breathing at a controlled rate and it is demonstrated that the breathing rate can be robustly estimated from the recorded pressure signal. The results suggest that the novel PSM technology can be used in clinical settings 
for non-obtrusive patient monitoring, with advantages related to low cost, very low noise, and robust linearity of measurements. 


\section{Acknowledgements}

I would like to thank my wife, Eli Angela Dalla Valle, for all the support and patience throughout my master's study. Without her unconditional help and support it would not be possible to even start this important step in my career.

Special thanks to Dr. James R. Green, who guided me through the program and provided all the important insights for the master's program and also during my stay at Carleton University. The support in our meetings and by email was essential to the advancement of this research. Finally, thank you for considering international students and for fighting for their academic successes.

Special thanks to Dr. Rafik Goubran, who guided me into the research world and provided opportunities to work with world-class research agencies such as AgeWell. Also for guiding me throughout the courses and challenges of the academic life, giving me support and advising me on the path of knowledge.

This study was supported by the Natural Sciences and Engineering Research Council of Canada. This work was supported by AGE-WELL NCE Inc., a member of the Networks of Centres of Excellence program, a Government of Canada program that funds partnerships between universities, industry, government and not-for- profit organizations. 


\section{Statement of Originality}

This thesis presents the work of the author, under the supervision of Dr. James R. Green and Dr. Rafik A. Goubran. This work was completed at Carleton University for the degree Master of Applied Science in Electrical and Computer Engineering with Specialization in

Data Science. Some of these results have been or will be presented in conference publication:

1. R. Selzler, J. R. Green, R. Goubran and F. Knoefel, "Developing a pressure sensitive mat using proximity sensors for vital sign monitoring," 2018 IEEE International Instrumentation and Measurement Technology Conference (I2MTC), Houston, TX, 2018, pp. 1-5.

This conference paper describes the initial design and validation of the pressure sensitive mat presented in this thesis. This work was expanded and improved through the analysis and evaluation of metrological properties presented in Chapter 4. This paper was presented by the author at the 2018 IEEE International Instrumentation and Measurement Technology Conference (I2MTC) in Houston - TX, US 


\section{Table of Contents}

ABSTRACT ......................................................................................................................II

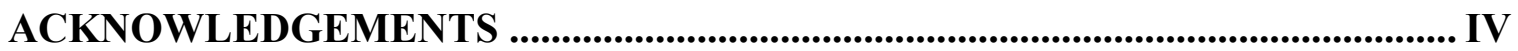

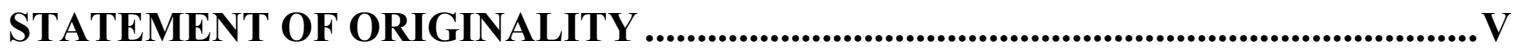

TABLE OF CONTENTS ............................................................................................ VI

LIST OF TABLES

LIST OF FIGURES ….................................................................................................... IX

LIST OF ABBREVIATIONS ....................................................................................... XI

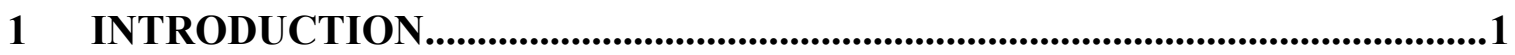

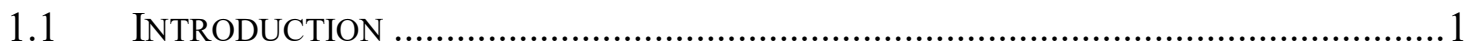





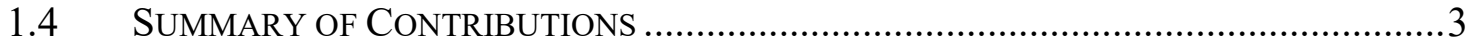

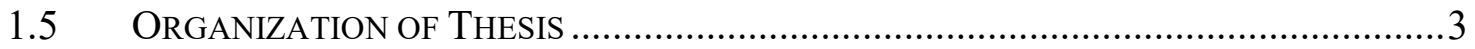

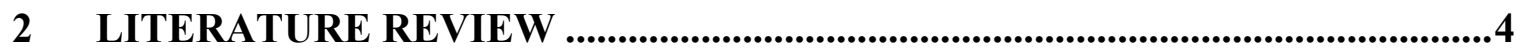





2.2 PRESSURE SENSITIVE MAT TECHNOLOGY ……….........................................

2.2.1 Force sensitive resistor PSM technology ...............................................

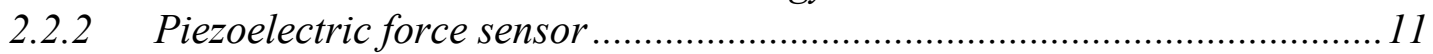

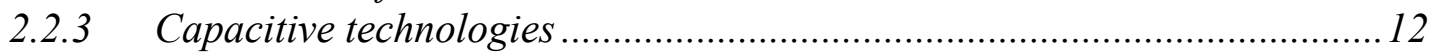

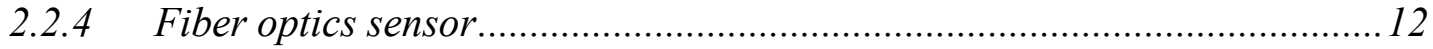



2.2.6 Relation of the proposed device to the state of the art................................17

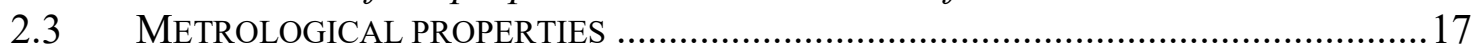

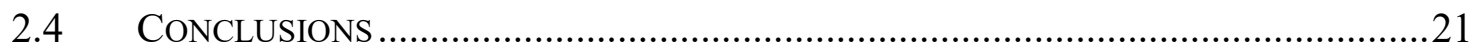

3 DESIGN OF A NOVEL PRESSURE SENSOR ......................................................23

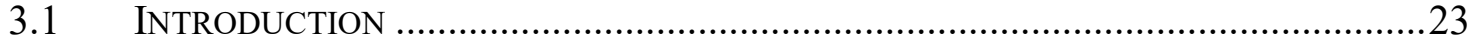

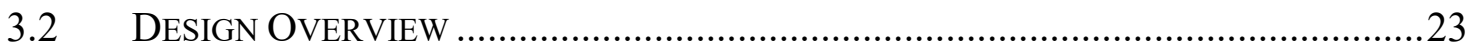



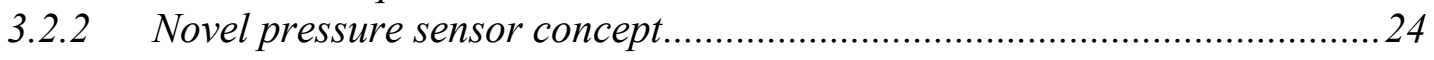

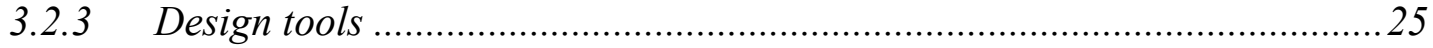

3.2.4 Design of Proximity-based Pressure Sensitive Mat ……………...............27

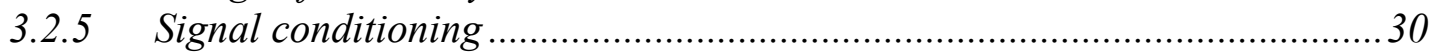





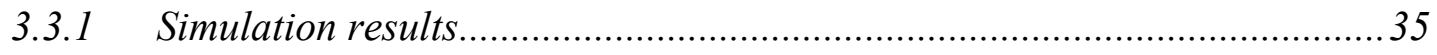




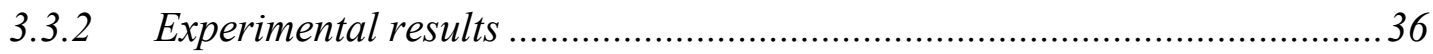

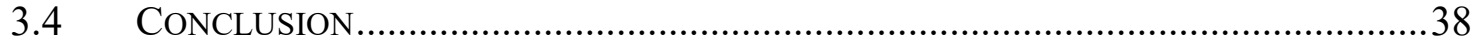

4 EVALUATION OF METROLOGICAL PROPERTIES .................................40

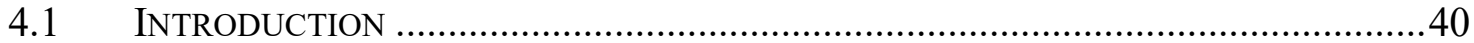

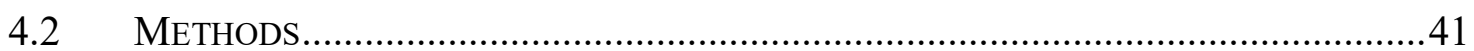

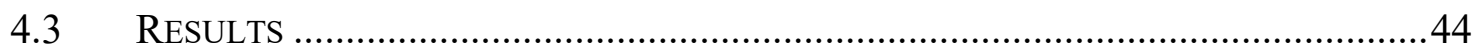

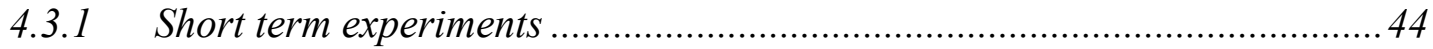

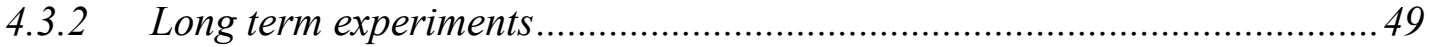

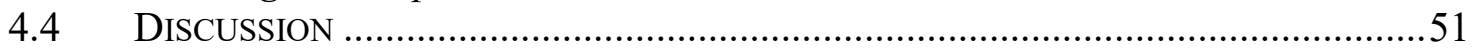

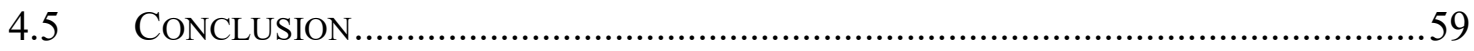

5 EVALUATION OF BREATHING RATE ESTIMATION USING THE



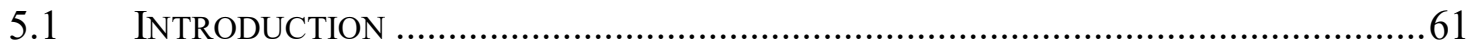

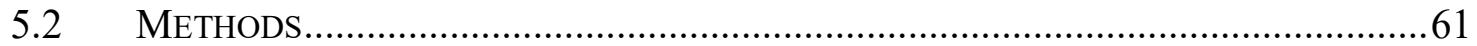

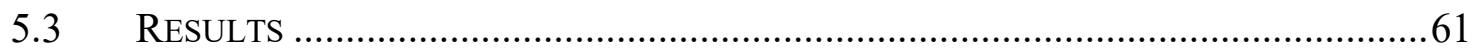

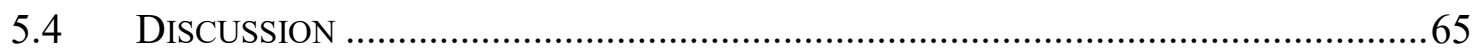

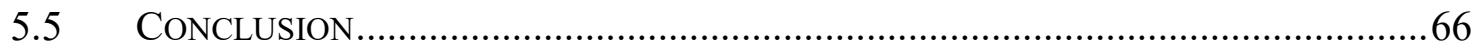

6 ANALYSIS OF AN ALTERNATIVE COMMUNICATION PROTOCOL ......68

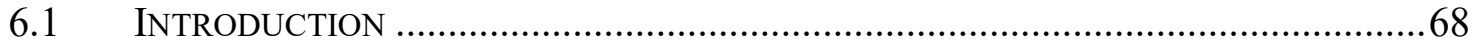

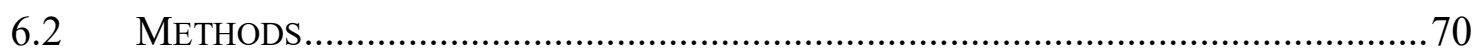

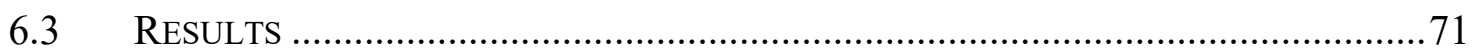

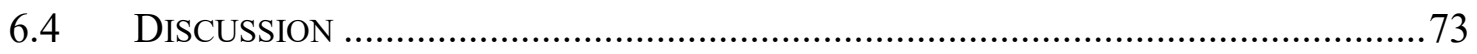

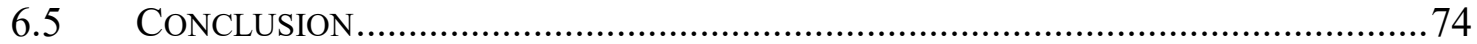

7 THESIS SUMMARY AND FUTURE RECOMMENDATIONS.......................75

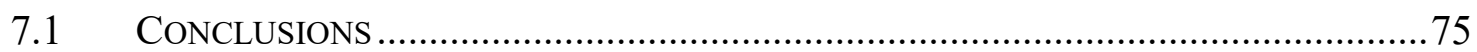

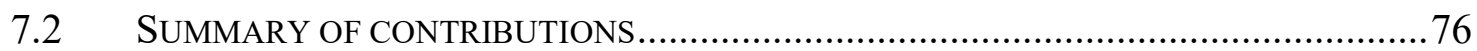

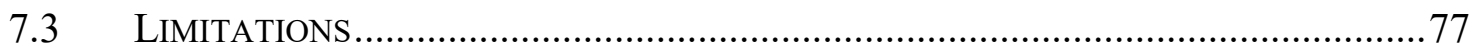

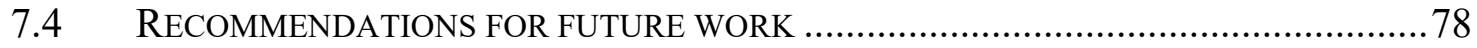

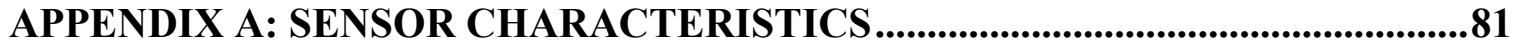

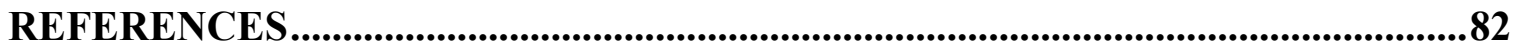




\section{List of Tables}

TABLE 1 - COMPARISON OF METROLOGICAL PROPERTIES BETWEEN THE PROPOSED "DOME" SYSTEM AND OTHER COMMERCIALLY AVAILABLE TECHNOLOGIES USING THE LIGHT WEIGHT ...

TABLE 2 - COMPARISON OF METROLOGICAL PROPERTIES BETWEEN PROPOSED "DOME" SYSTEM AND OTHER COMMERCIALLY AVAILABLE TECHNOLOGIES USING THE HEAVY WEIGHT 


\section{List of Figures}

FIGURE 1 - BODY PRESSURE MAPPING [42] 8

Figure 2 - CONSTRUCtion OF THE FLEXIForCE SENSOR FrOM TEKSCAN [45] 10

Figure 3 - PRESSURE SENSITIVE ARRAY USING FORCE RESISTIVE SENSORS [42] 11

FigurE 4 - ForCE VS. RESISTANCE AND CONDUCTANCE OF COMMON FORCE RESISTIVE SENSOR [44] 11

FIGURE 5 - WORKING PRINCIPLE OF FIBER OPTIC SENSOR [49] 13

FigURE 6 - KINOTEX SENSOR MAT [50] 13

FIGURE 7 - TACTILE SENSOR ELEMENT PROPOSED BY [9]. 14

Figure 8 - PROPOSED DESIGN OF A TACTILE SKIN AND ITS APPLICATION TO ROBOTICS [9]. 15

FIGURE 9 - PROPOSED DESIGN OF A PRESSURE SENSEL USING PHOTO SENSORS WHERE THE TRANSMITTED LIGHT IS MEASURED [51]. 16

FiguRE 10 - MEASUREMENT OF PRESSURE DISTRIBUTION OF INFANTS USING THE TECHNOLOGY PROPOSED BY DONATI ET AL. [52]. 16

FIGURE 11 - WORKING PRINCIPLE OF THE PROTOTYPE PROPOSED BY [8]. 17

FiguRE 12 - CONCEPT OF PRESSURE SENSITIVE CELL. A) NO PRESSURE IS APPLIED, AND B) PRESSURE IS APPLIED. 25

FIGURE 13 - BLOCK DIAGRAM OF THE IMPLEMENTED SYSTEM 28

Figure 14 - FLEXIBLE PRINTED CIRCUIT BOARD WITH A TIVATM BOARD LAUNCHPAD TM4C123G ATTACHED TO IT. THE BLACK RECTANGLES ON THE GREEN CIRCUIT BOARD ARE THE ARRAY OF PROXIMITY SENSORS.

FIGURE 15 - BOTTOM, TOP, AND FRONT VIEWS OF THE SILICONE ELASTIC LAYER. 30

FIGURE 16 - MAIN TAB OF THE GUI DEVELOPED TO COMMUNICATE WITH THE PROTOTYPE TO SET CONFIGURATIONS AND RECEIVE DATA.

Figure 17 - Multiple Sensors tab of the implemented GUI. EACH Line REPRESENTS THE VALUES READ FOR EACH SENSOR.

Figure 18 - Total Pressure tab of the IMPlEMENTEd GUI. EACH POINT IN THE LiNE REPRESENTS THE SUM OF THE VALUES READ FROM ALL THE SENSORS. 34

FIGURE 19 - IMAGE TAB OF THE IMPLEMENTED GUI. THE VALUES FROM THE SENSORS ARE INTERPOLATED TO GENERATE AN IMAGE BASED ON THE PRESSURE APPLIED TO EACH INDIVIDUAL CAVITY. THE COLOR MAP IS ADJUSTED BY THE USER IN THE SLIDER BELOW THE IMAGE.

FIGURE 20 - FEA SIMULATION FOR 0.1 (TOP LEFT), 0.2 (TOP RIGHT), 0.3 (BOTTOM LEFT) AND 0.4 (BOTTOM RIGHT) PSI APPLIED IN THE Y-AXIS. COLOURING REPRESENTS THE MAGNITUDE OF DISPLACEMENT.

FIGURE 21 - EXPERIMENT OF LINEARITY. A) INCREMENTAL WEIGHTS OF 100G ARE PLACED ON TOP OF A CIRCULAR SURFACE OVER A PERIOD OF 1 MIN AND REMOVED FROM THE SURFACE FOR 1 MIN. B) GRAPH SHOWS THE APPLIED PRESSURE IN THE X-AXIS AND THE MEASURED PRESSURE IN THE Y-AXIS.

FiguRE 22 - COMMERCIALLY AVAILABLE SENSORS USED IN THIS STUDY. LEFT: XSENSOR, MIDDLE: S4, AND RIGHT: TEKSCAN

FIGURE 23 - SHORT TERM EXPERIMENT FOR FOUR DIFFERENT PSM TECHNOLOGIES. WEIGHTS ARE ADDED ON TOP OF THE PSM FOUR TIMES FOR ONE MINUTE EACH, WITH UNLOADED INTERVALS OF ONE, TWO AND THREE MINUTES. 
FIGURE 24 - AVERAGE PRESSURE OVER FIFTY SECONDS WITH WEIGHTS APPLIED FOUR TIMES BETWEEN UNLOADED INTERVALS OF ONE, TWO AND FIVE MINUTES IN THE SHORT-TERM EXPERIMENT. (A) LIGHT AND (B) HEAVY WEIGHT APPLIED TO THE PRESSURE MEASUREMENT SYSTEM.

FIGURE 25 - NORMALIZED AVERAGE PRESSURE FOR LIGHT AND HEAVY WEIGHT IN SHORTTERM EXPERIMENTS.

FIGURE 26 - CHANGE IN CREEP OVER TIME USING WINDOW SIZE OF ONE HOUR.

FIGURE 27 - PERCENTAGE DRIFT OVER 11 HOURS FOR THE LIGHT WEIGHT (LEFT) AND HEAVY WEIGHT (RIGHT)

FIGURE 28 - SHORT TERM EXPERIMENT REPEATED THREE TIMES FOR THE TEKSCAN EQUIPMENT

Figure 29 - NORMALIZED AVERAGE PRESSURE FOR TEKSCAN EQUIPMENT OVER THREE TRIALS.

FIGURE 30 - RISE TIME ANALYSIS OF THE FOUR TECHNOLOGIES FOR LIGHT WEIGHT (TOP) AND HEAVY WEIGHT (BOTTOM)

FIGURE 31 - RISE TIME ANALYSIS USING THE FILTERED SIGNALS FOR THE FOUR TECHNOLOGIES FOR LIGHT WEIGHT (TOP) AND HEAVY WEIGHT (BOTTOM) 58

FIGURE 32 - TOTAL PRESSURE OVER TIME OF A SUBJECT LAYING ON THE PSM PROTOTYPE IN SUPINE POSITION. RESPIRATION RATE WAS CONTROLLED WITH A METRONOME. THE BREATHING RATE OF THE TOP FIGURE WAS SET TO 12 BREATHS PER MINUTE AND THE BOTTOM FIGURE WAS SET TO BE 20 BREATHS PER MINUTE.

FIGURE 33 - FILTERED TOTAL PRESSURE OVER TIME OF A SUBJECT LAYING ON THE PSM PROTOTYPE IN SUPINE POSITION. RESPIRATION RATE WAS CONTROLLED WITH A METRONOME. THE BREATHING RATE OF THE TOP FIGURE WAS SET TO 12 BREATHS PER MINUTE AND THE BOTTOM FIGURE WAS SET TO BE 20 BREATH

FigURE 34 - BREATH INTERVALS OF A SUBJECT LAYING ON THE DEVELOPED PROTOTYPE IN SUPINE POSITION. SIGNALS EXTRACTED FROM THE ORIGINAL SIGNALS AND FROM THE FILTERED SIGNALS USING A 1 S RECTANGULAR WINDOW MOVING AVERAGE. 65

FIGURE 35 - CIRCUIT REPRESENTATION OF THE PROPOSED COMMUNICATION PROTOCOL. 71

FiguRE 36 - EFFICIENCY COMPARISON BETWEEN THE I2C AND ALTERNATE PROTOCOL CONSIDERING ONE, TWO, AND TEN BYTES READ.

FiguRE 37 - Fold INCREASE BETWEEN THE ALTERNATE AND I2C PROTOCOL CONSIDERING THAT ONLY TWO BYTES ARE BEING READ PER SENSOR.

FIGURE 38 - RELATIVE RADIANT INTENSITY VS. ANGULAR DISPLACEMENT OF THE EMITTER (REPRODUCED FROM [57])

FIGURE 39 - RELATIVE RADIANT SENSITIVITY VS. ANGULAR DISPLACEMENT OF THE PROXIMITY SENSOR (REPRODUCED FROM [57]) 


\section{List of Abbreviations}

\begin{tabular}{|l|l|}
\hline Abbreviation & Definition \\
\hline bpm & Beats per minute \\
\hline CAD & computer-aided design \\
\hline CoV & Coefficient of Variation \\
\hline GUI & Graphical User Interface \\
\hline HR & Heart rate \\
\hline IMU & Inertial Measurement Unit \\
\hline MICU & Medical Intensive Care Unit \\
\hline NTP & Normalized total pressure \\
\hline PCB & Printed Circuit Board \\
\hline PSM & Pressure Sensitive Mats \\
\hline PSA & Pressure Sensitive Array \\
\hline PVDF & Polyvinylidine fluoride \\
\hline RR & respiration rate \\
\hline SCL & serial clock line \\
\hline SDA & serial data line \\
\hline SP & sensor pressure \\
\hline TP & total pressure \\
\hline
\end{tabular}




\section{Introduction}

\subsection{Introduction}

This chapter details the motivation for this thesis, reviews and states the problem, provides a summary of contributions, and describes the organization of the thesis structure.

\subsection{Motivation}

Pressure sensitive mats (PSM) or pressure sensitive arrays (PSA) are being used to measure contact force distribution in many fields. Current products are able to measure a wide range of applied forces, ranging from the high-pressure profile exerted by a car tire [1] to the low-pressure profile exerted by spray equipment where the amount of fluid deposit on the surface is measured by pressure [2].

Current PSM technologies have been used for non-obtrusive patient monitoring. Specifically, PSMs have been used to monitor the bed occupancy of older adults in long-term monitoring [3], to monitor the ulcer of patients in a Medical Intensive Care Unit (MICU) [4], and also by the research community in experiments for body mass changes over time [5] and fluid intake monitoring [6]. Current technologies can extract useful information from pressure mapping distributions, but improved sensor characteristics could provide better estimates of pressure profiles in which higher signal quality and reliability could provide more features from the measured signals. Thus, developing novel sensors with improved metrological properties will lead to improved patient monitoring.

PSA have been implemented using different technologies for use in fields such as scientific measurements and biomedical research. However, as discussed in the next chapter, each sensing 
technology has its metrological limitations. Furthermore, the cost of currently available pressure sensitive mats can be thousands of dollars, largely limiting their use in small-scale research. While in research the number of subjects and the duration of data collection are small, in clinical setings the number of patients is greater and the required time for patient monitoring is commonly much longer.

New pressure-sensing technologies have recently been introduced in the field of robotics [7]-[11], where the sense of touch is important for object manipulation. However, these technologies have not been explored in a PSM configuration suitable for data acquisition for nonobtrusive patient monitoring. Also, there is still a need for improved pressure-sensing technologies with affordable prices, which could be used in both clinical or home monitoring settings.

Over the last decades, the cost of integrated circuits have been continuously decreasing, and the quality, vis-à-vis noise and reliability, of such circuits has significantly increased. One important advance is related to proximity sensors, which use modulated infrared light with an embedded emitter and receiver, as well as integrated communication protocols, in a single miniaturized and integrated package. This thesis examines the combination of such a sensor with a compressible chamber so as to transduce changes in applied pressure into changes in detected proximity. A custom flexible circuit is designed and tested to achieve a prototype PSM using this new pressure-sensing technology.

\subsection{Problem Statement}

We seek to develop a novel pressure-sensitive mat using solid-state infrared sensors to achieve improved metrological properties, suitable for low-cost unobtrusive patient monitoring. 


\subsection{Summary of Contributions}

Three principle contributions are made in this thesis:

1) Developed novel pressure sensor based on a solid state infrared proximity sensor that is suitable to deploy as a PSM.

2) Evaluated metrological properties of the prototyped device, demonstrating improved performance relative to commercially available pressure sensitive mats.

3) Demonstrated suitability for patient monitoring by estimating the breathing rate using pressure measurements.

\subsection{Organization of Thesis}

This thesis consists of 7 chapters. Chapter 2 briefly discusses the current pressure sensitive technologies available on the market and previous applications of pressure-sensitive sensors with emphasis on patient monitoring. In Chapter 3, we describe the development of the prototype sensor. Chapter 4 details the experimental determination of the metrological properties of the novel sensor. It also describes initial experiments to demonstrate that the novel sensor is suitable for patient monitoring. Chapter 5 describes the proof-of-concept experiment whereby breathing rate is estimated using the prototype sensor. In Chapter 6, the communication protocol is analyzed and a new protocol is proposed with focus on ease of manufacturing and efficiency. Finally, Chapter 7 presents a summary of contributions and provides recommendations for future work. 


\section{Literature Review}

This chapter discusses remote patient monitoring with emphasis on eldercare, the current technologies used to manufacture pressure sensitive mats, an overview of the metrological characteristics of pressure sensitive mats, as well as the characteristics considered in the design of the proposed technology.

\subsection{Remote Patient Monitoring}

As society evolves, the age distributions of the population changes. It is known that the rate in which people are getting older in Canada is higher than the rate in which people are born. According to the 2010 Canada Yearbook [12], one out of seven Canadians are 65 years old or older. Since the sixties, the senior population has been steadily growing, from $8 \%$ in 1960 to $14 \%$ in 2009 [12]. The median age of the Canadian population in 1971 was 26.2 years old, and it is expected to be $42-45$ years old in 2036, and $42-47$ in 2061 [12]. As the population ages, there is an increasing need to monitor patients in an efficient way and at a lower cost. Clearly, technology advancements have the potential to decrease the cost of remote patient monitoring [13].

Non-obtrusive patient monitoring has been the focus of many research teams across the globe. Increasing interest in technologies to measure a patient's vital signs without the use of sensors attached to the subject's body is expanding because it brings comfort to the patient and also allows the monitoring of the patient's signals without specialized personal and facilities. Also, the reliability of the collected data does not depend on the subject remembering that the sensors must be worn.

In a recent study, Wallace et al have fused multiple sensor modalities for the measurement and classification of overnight wandering by dementia patients using multi-sensors [14]. Low cost 
consumer home automation sensors available on the market were used to monitor and detect wander, as well as give feedback to the subjects, suggesting further safety actions. In this study, a number of different sensor modalities were utilized. Infra-red motion sensors were used to detect the position of the patients in different rooms of a house. Bed sensors are used to detect bed occupancy. Latch sensors are used to monitor if doors are closed or opened. A speaker and smart light are used to give feedback to the patient through sounds, or illuminating a path, directing the subject back to the bedroom in the case of late night wandering [14].

The use of such technology in a controlled environment can help with the monitoring of patients and provide useful information for medical care. For example, doctors and systems could detect the number of times a given patient is getting out of the bed and which times, helping to promote a better diagnose of the illness. This study demonstrates that patient monitoring can be done at home and inexpensively.

Another recent study focused on fall detection in the senior population [15]. Between $20 \%$ and $30 \%$ of seniors fall each year, affecting not only the seniors, but also the family members, care providers, and the health care system. Falls are the leading cause of injury-related hospitalization among the elderly population in Canada. It can lead to negative mental conditions such as fear of falling, confusion, loss of autonomy and isolation, immobilization and depression [15]. Fall detection is one of the important factors to monitor the health of patients. By detecting the fall, a system can call the emergency service response, which may accelerate the attendance by first responders, thus minimizing the consequences of the fall. Therefore, it is important to predict falls and minimize the unfortunate outcomes from such accidents. The use of smartphones to detect falls using accelerometers have been proposed in a number of studies [16], [17]. However, it is unlikely that long term monitoring would be possible, considering that the subjects would have to 
carry their phones all day long. As an alternative to phones, wearable sensors such as the Fitbit and Apple Watch can be used to track accelerations of the patient.

Most wearable sensors available on the market today have integrated Inertial Measurement Units (IMUs) or accelerometers, as well as multiple other sensors. These devices are used to monitor a subject's activities and health related data. Steps walked and climbed, heart rate, quality of sleep, blood oxygenation, and other personal metrics are examples of signals collected. One limitation of such wearable sensors is that they must be recharged periodically. In the case of patient monitoring, it is important to record these signals constantly, which may not be the case when the subject forgets to recharge these devices.

Other than wearable sensors, a wide range of sensor modalities have been explored for fall detection, including camera systems [18]-[21], acoustic/vibration-sensor based systems [22], [23], radar systems [24], [25], and a barometric pressure sensor combined with accelerations [26]. By using different sensors, it is possible to improve the performance of a classification system, avoiding false positives and false negatives.

\subsubsection{PSM for Patient Monitoring}

Pressure sensitive mats have been used in a number of patient monitoring studies. Pressure sensitive mats have been proposed to detect falls in the elderly population [27]-[29]. Piezoresistive sensors were used to detect falls in a range of scenarios, reaching sensitivity and specificity of $88.8 \%$ and $94.9 \%$ respectively [27]. Accuracy of $100 \%$ was reached to differentiate standing from lying postures of a subject using fiber-optic sensors [28]. Pressure sensitive mats can also be used to monitor whether a person is laying on a bed or not, which provides extra information to other non-obtrusive systems. Effective bed occupancy detection has already been demonstrated in other studies [3], and can be simply added to a multi-sensor system. 
In addition to fall detection and bed occupancy, other health conditions can also be monitored by pressure sensitive mats. For example, when a patient remains in the same position in bed for a prolonged period, pressure sores or ulcers can form in certain conditions. Pressure sensitive mats can monitor both the intensity and duration of contact points, thus providing information to hospital staff who reposition the patient, reducing the effects of pressure on human body [4].

Some researchers are considering long term monitoring using pressure sensitive mats to diagnose or monitor chronic diseases. One of the advantages of long term monitoring is that rare events can be found through signal processing and pattern classification. It is not always possible to verify these events when a patient is taking measurements for a few hours, but it may be possible if the patient has a data history continuously recorded during weeks. In one such study, it was demonstrated that pressure sensitive mats can be used to monitor the distribution of fluids throughout the body while laying on a mattress, which can provide useful information about illness such as congestive heart failure, nocturia and kidney failure [6], [30], [31]. Other physiological measurements such as heart rate [32]-[34], and breathing rate [35], [36] have been successfully extracted using pressure sensors. Sleep apnea has been measured using both load cells [37] and using PSM [38], [39]. The stability of subjects can also be evaluated using the center of pressure measured by PSM [40], [41]. As can be seen, pressure sensitive mats can be used in a wide range of applications where non-obtrusive patient monitoring is needed. Further reductions in cost and improvements in the metrological properties of PSM will improve these applications and enable novel applications of PSM to non-obtrusive patient monitoring. 


\subsection{Pressure sensitive mat technology}

Pressure sensitive mats are devices that measure the pressure applied across the sensor. These devices usually are arranged in a matrix distribution, where each sensor cell, or sensel, measures the pressure applied to the sensor. The pressure applied to a PSM can be visualized by associating each sensel with a pixel of an image, where the colour or intensity of the pixel communicates the magnitude of applied pressure. Figure 1 shows a color map representation of the pressure distribution of a body laying atop a mattress and a PSM. The silhouette of the subject's body can be clearly seen, where the red color represents high pressure applied and blue areas represent pressures of low intensities. It is possible to analyse which parts of the body have stronger contact with a given surface. It this force is too strong in a given area, the patient might be advised to change position, thus avoiding pressure ulcers, or the surface where the patient is lying can be designed in a new way that will better support the patient providing more comfort and reducing contact pressure by better distributing the load.

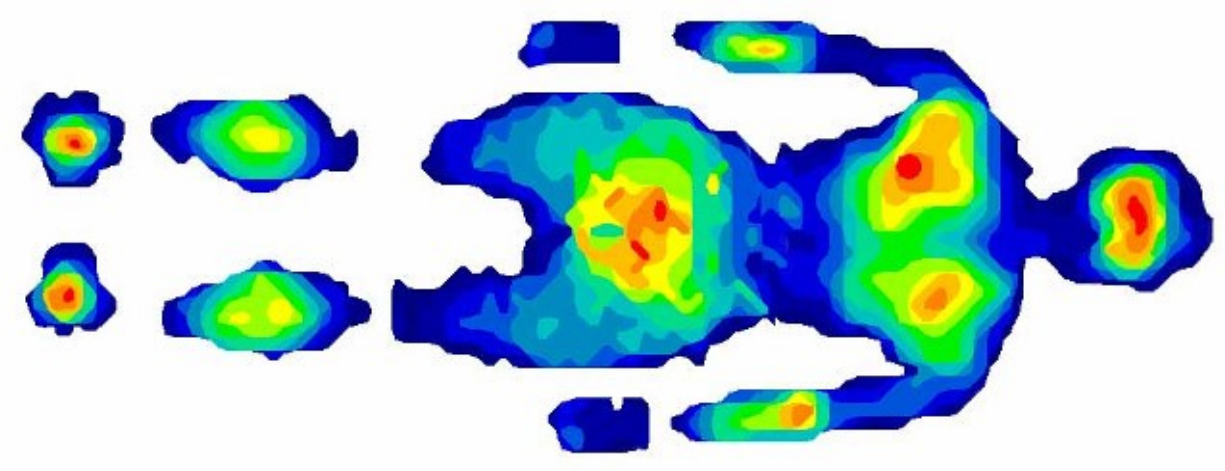

Figure 1 - Body pressure mapping [42]

The change in pressure distribution can be measured over time, which means that the body movement can be quantified. For example, when a person breathes, the weight distribution of the 
body will change as the lung inflates and deflates. Thus, with signal processing of the PSM data, it is possible to estimate the breathing rate [43]. Also, it is possible to visualize time-varying changes in pressure distribution across the body. For example, fluid retention in the legs could be quantified over night and be used to identify congestive heart failure. Similarly, the authors in [6] presented a way of monitoring the fluid intake and bladder voiding using pressure sensitive mats, where the fluid distribution on the body is analysed.

The remainder of this section will review the main PSM technologies and their principles. These include force sensitive resistors, piezoelectric force sensors, capacitive sensors, and optical sensors.

\subsubsection{Force sensitive resistor PSM technology}

Force resistive sensors comprise two layers of thin-film polymer. Each of these layers contains a conductive pressure sensitive element printed on a conductor. The ends of the polymer layers are connected together. When there is no load being applied across the sections, the sensor has a very high resistance in the range of Megaohms. When force is applied across these sections, an elastic deformation of the polymer occurs, causing a decrease in resistance, typically in the range of kiloohms [44]. Figure 2 shows the construction of a force sensor made by Tekscan. The silver ends conduct the electrical signals from one end to another, enabling the measurement of the resistance across the pressure sensitive ink. The concept of force sensing can be extended to PSM, where each sensel of the array is a force sensor. In fact, resistive pressure sensitive mats are based on force sensors, and the basic structure of each sensel is the same as an individual force sensor. 


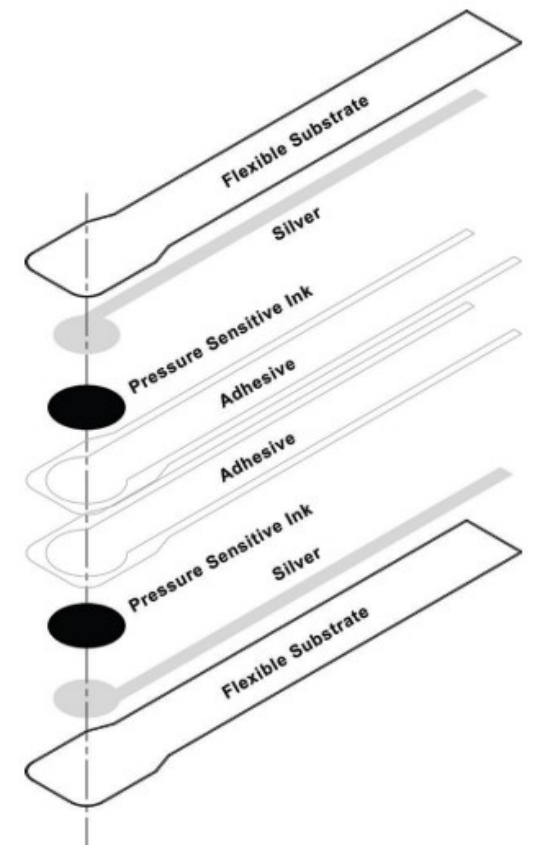

Figure 2 - Construction of the FlexiForce Sensor from Tekscan [45]

Figure 3 shows an array of sensing points manufactured by Tekscan [42]. Each of black point is one sensel, which allows the measurement of the pressure distribution of the human body. Figure 4 shows the usual pattern of the resistance and conductance of force resistive sensors when force is applied across the sections of the sensor [44]. The conductance of this sensor is linear and proportional to the force applied across the sections of the sensor. 


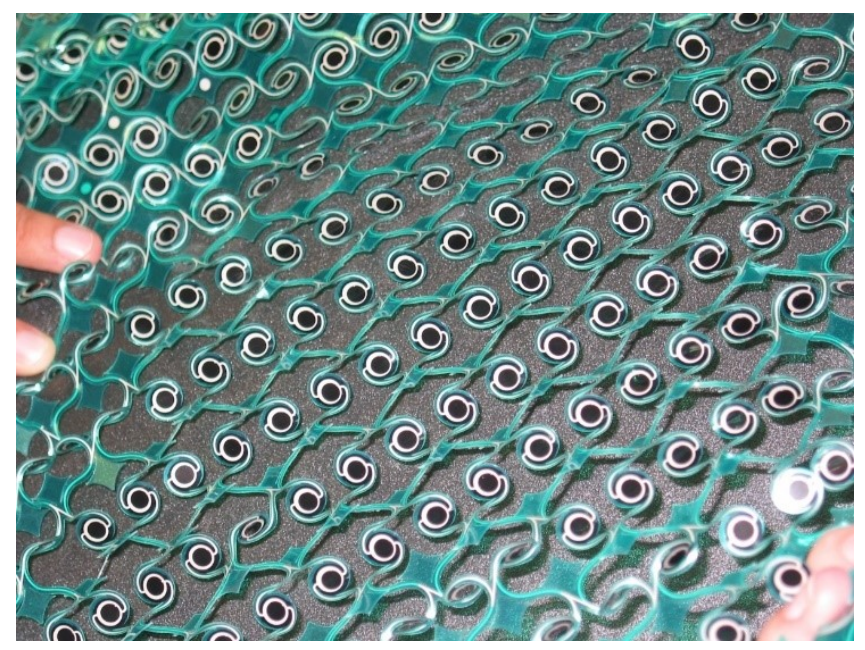

Figure 3 - Pressure sensitive array using force resistive sensors [42]

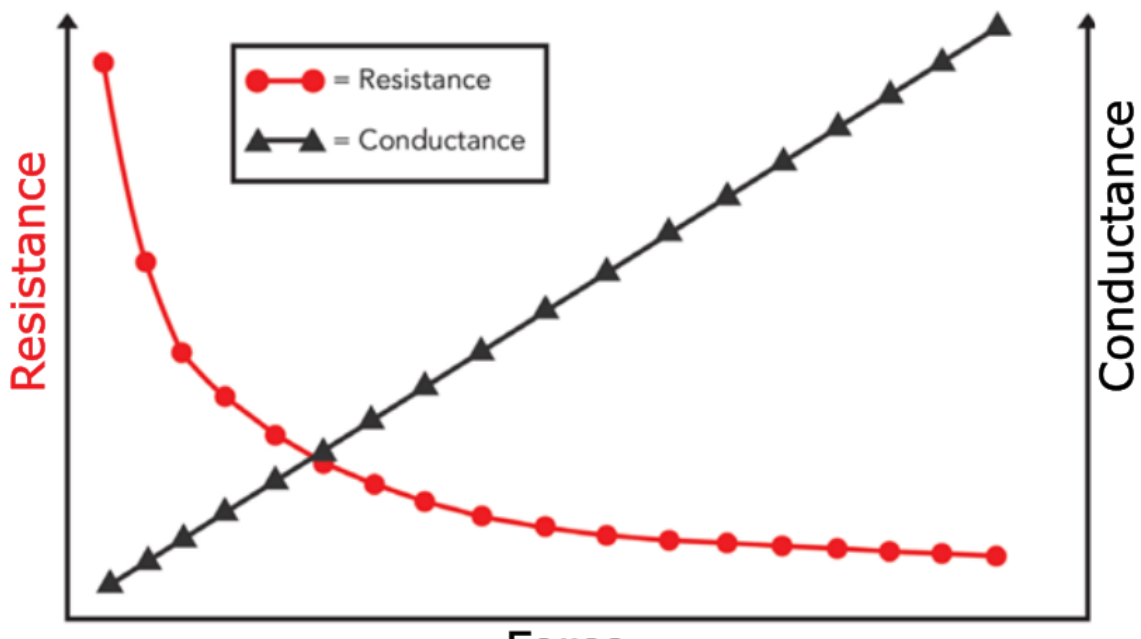

Force

Figure 4 - Force Vs. resistance and conductance of common force resistive sensor [44]

\subsubsection{Piezoelectric force sensor}

Piezoelectric force sensors use the piezoelectric effect to measure force by converting the mechanical stress into electrical charges. The piezoelectric effect can be observed in materials such crystals, ceramic materials, and particular polymers. One example of polymers used in pressure sensors is the Polyvinylidine fluoride (PVDF), which is made of thin and flexible foil material 
[46]. Piezoelectric sensors generate small electrical charges when mechanical stress is applied to them. In pressure sensors, the charge is amplified and converted to force.

\subsubsection{Capacitive technologies}

A number of studies have presented the use of capacitive technology to measure the pressure applied across two sections [29], [47], [48]. Usually this type of configuration consists of two aligned electrodes separated by a thin layer of compressible material. When force is applied across the section of the electrodes, the material is compressed, causing the electrodes to become closer. As the distance between the electrodes decreases, there is an increase in capacitance. This change in capacitance can be measured as a proxy for applied pressure. An array configuration of such sensors allows the creation of a PSM for the measurement of a pressure profile of forces applied over a given surface.

\subsubsection{Fiber optics sensor}

As shown in Figure 5, the working principle of fiber optic sensors is based on the modulation of the fiber-guided light [49]. The source light is sent from one extremity of the optical fiber and is measured by the detector at the other extremity. The properties of the transmitted light will be altered on the way, depending on applied pressure. One of the common properties measured is the intensity of the measured light. The Kinotex sensor uses an LED as source of light, the foam is used to interact with the light, and a receiver to detect the intensity of light at the end of the fiber. When force is not being applied to the foam, a given light intensity is measured, but when the pressure is applied to the foam, there is a decrease in light intensity [50]. Figure 6 shows the common application of the Kinotex pressure sensor mat, which uses multiple fibers arranged in a matrix form, providing the pressure profile of the applied forces. 


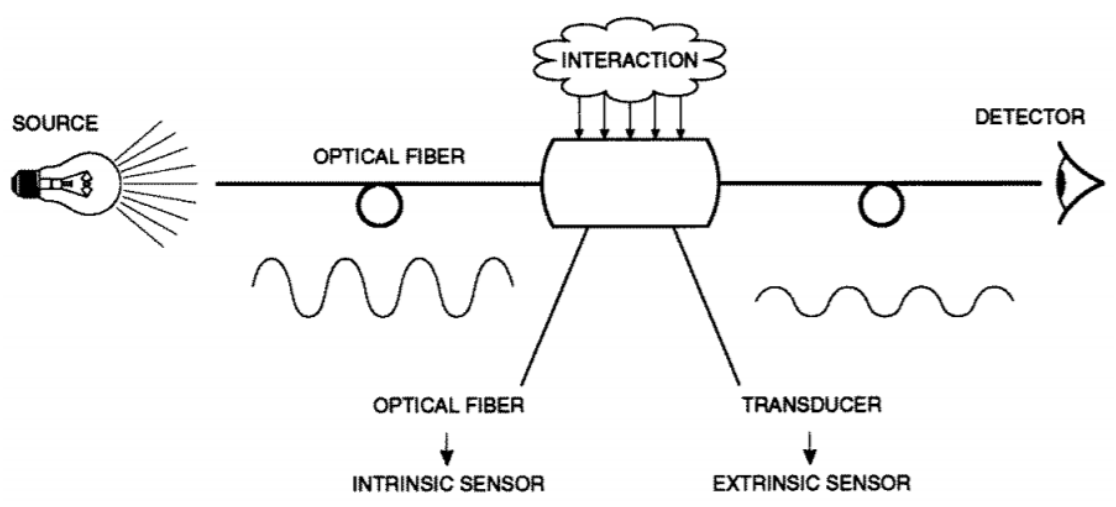

Figure 5 - Working principle of fiber optic sensor [49]

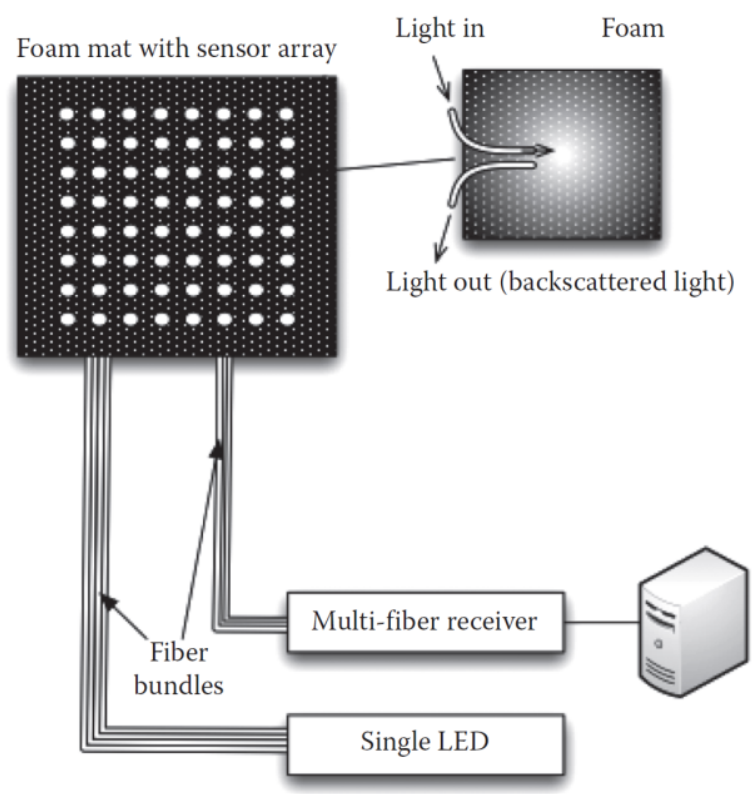

Figure 6 - Kinotex sensor mat [50]

\subsubsection{Other PSM technologies}

A number of technologies have been introduced in research papers using variations of the technologies discussed above. This thesis is based on infrared proximity sensors which measure the distance from the sensor to the top of a compressible cavity. 
The design of a sensor "skin" in flexible surfaces using optical sensors was proposed by Ohmura et al in [9]. In this study, a compressible urethane foam is positioned on top of an array of LEDs and photo detectors. As pressure is applied to the foam, the light radiated from the LED is scattered, causing a change on the signal measured by the photodetector, which is then translated to the force applied in each sensel. Figure 7 shows the concept behind one sensel proposed in [9]. The disposition of the sensels are shown in Figure 8. As can be seen, the sensors can be glued to irregular surfaces such as the arms of robots, enabling a sense of touch.



Figure 7 - Tactile sensor element proposed by [9]. 


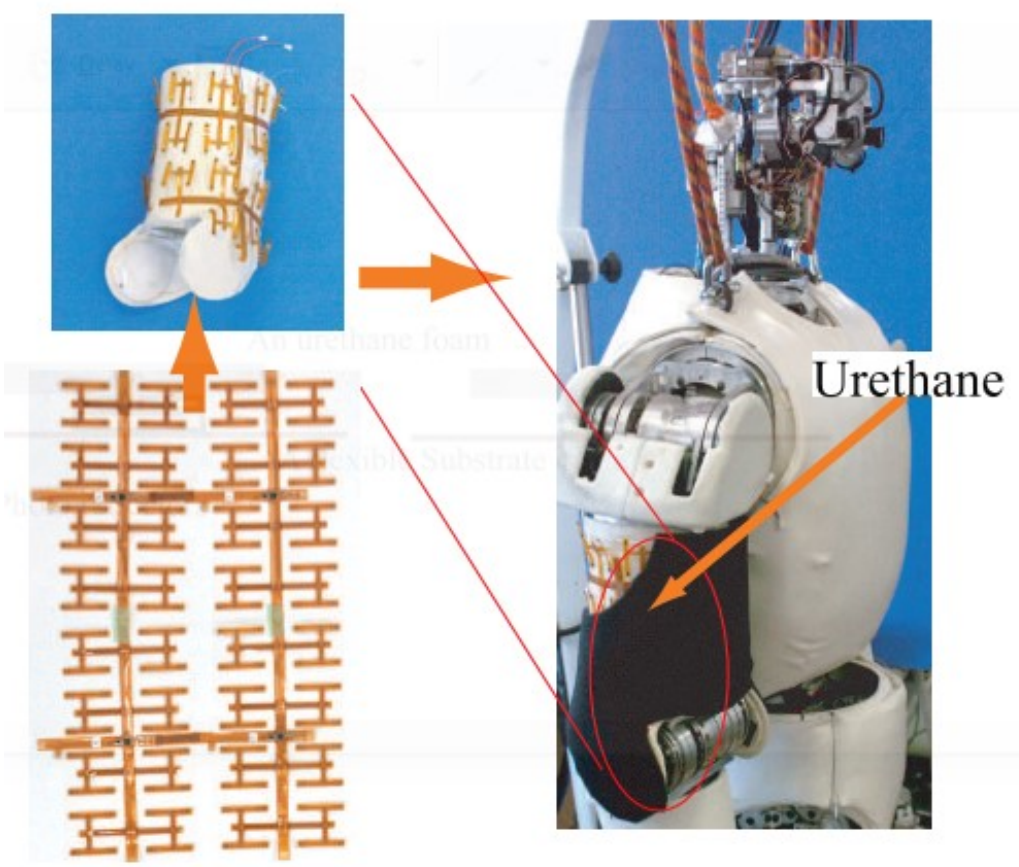

Figure 8 - Proposed design of a tactile skin and its application to robotics [9].

The use of photo sensors were also proposed by Donati et al. in [51]. They have proposed positioning the emitter on the horizontal axis, whereas the receiver is on the vertical axis. The medium between the emitter and the receiver is air, and they are surrounded by a compressible cavity with a blocking element in the middle. Figure 9 illustrates the concept for one sensel, which can be distributed in different forms across a surface, depending on the design. When force is applied to the cavity a deformation will occur, causing the cavity to move vertically to the direction of the printed circuit board. This deformation causes the blocking element to decrease its distance to the PCB, blocking part of the light emitted by the transmitter. Thus, the light measured at the receiver will be smaller, and this can be translated to applied force. The authors, presented the application for robotic uses, therapeutic assessments, and fitness applications [51], where the proposed design is worn by patients around the limbs and thorax, or on the sole of the shoes. In another study, they presented the use of the same technology to predict the posture of infants using 
the measurements of pressure distribution [52]. Figure 10 shows the measurement of pressure distribution of an infant in different positions using the technology developed by Donati et al.


Figure 9 - Proposed design of a pressure sensel using photo sensors where the transmitted light is measured [51].
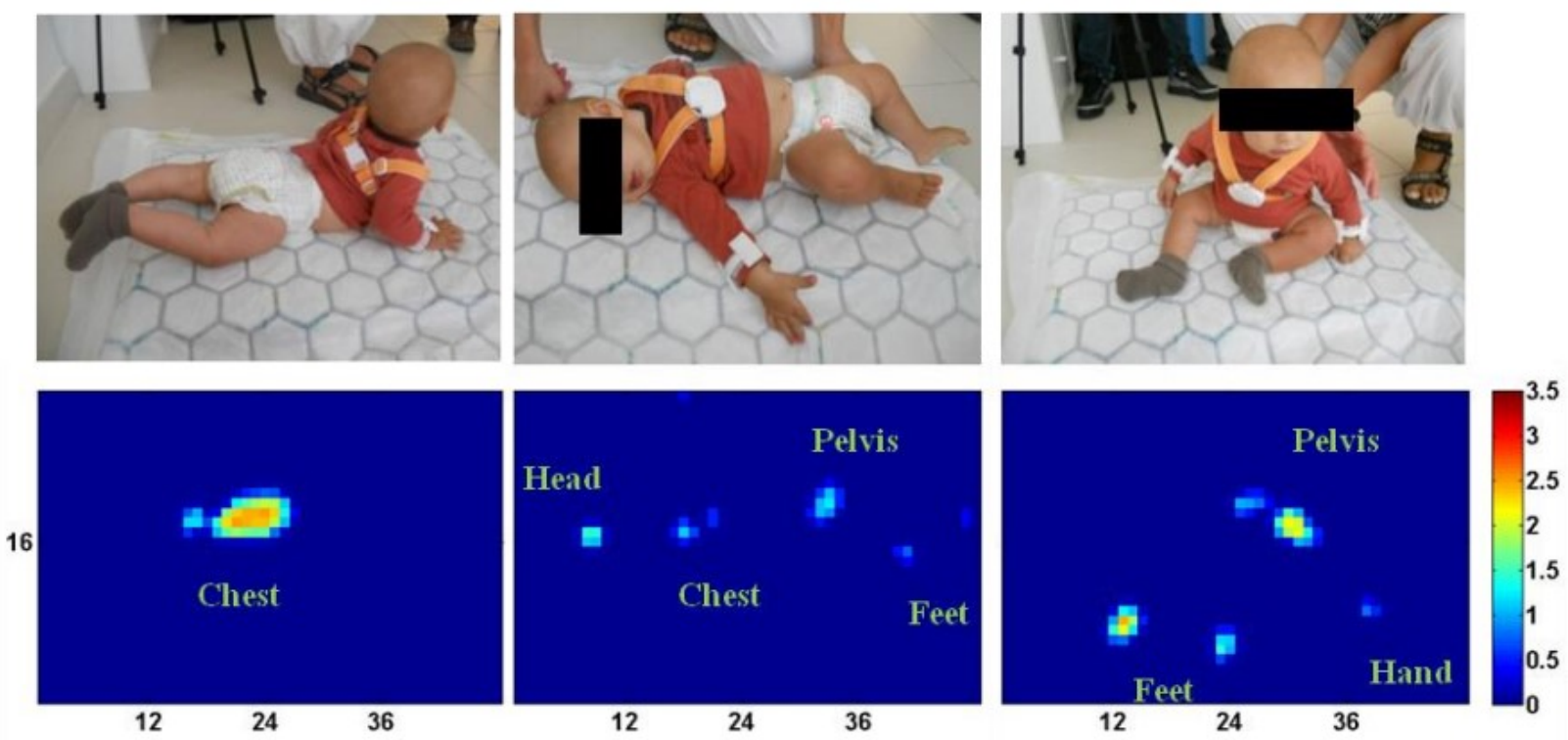

Figure 10 - Measurement of pressure distribution of infants using the technology proposed by Donati et al. [52].

The use of photo sensors to measure pressure in robotics applications were also proposed by De Maria et al. in [8]. In this design, both emitter and receiver are positioned in the vertical axis, covered by a cavity. Sixteen sensels are positioned in an array configuration, with rectangular 
cavities on top. The surface of this cavity is oval, simulating the tip of a robotic finger. Figure 11 shows the sketch of the working principle proposed in [8]. They were able to quantify the force and torque applied to the mechanism. By interpreting the changes in light intensity across the array of cavities in the device, it can operate as a six-axis force/torque sensor for feedback control in robotic systems.



Figure 11 - Working principle of the prototype proposed by [8].

\subsubsection{Relation of the proposed device to the state of the art}

Of the technologies discussed above, the design proposed in this thesis is conceptually most similar to that proposed in [8]; however, we use a different proximity sensor, create a prototype PSM as opposed to a robotic finger, and explore its suitability for unobtrusive patient monitoring.

\subsection{Metrological properties}

As technology evolves, new electronic components are offered by the industry including novel sensors for quantifying a given measurand, such as pressure, temperature, humidity, and others. It is important to know how well a given sensor works in relation to existing technologies, so that 
the system designers can make informed decisions. The metrological properties numerically represent how well a sensor performs. Whenever such decisions affect people's lives, greater care should be taken to ensure system reliability. Thus, it is even more important to know the sensor's characteristics when the final application will be related to vital signals monitoring. Multiple experiments must be run for a given sensor, so that its characteristics are well specified. For example, short term experiments can verify the repeatability of the measurements over time, estimating the uncertainty associated with the measurement, and providing information about how precise the sensor will perform in repeated measurements using the same experimental scenario. On the other hand, long term experiments can quantify the differences in the measurements over extended periods of use, providing information about the deviations that occurs over time when the scenario of the experiment does not change. For example, if a fixed weight is put on top of a pressure sensor for long periods of time, deviations occurring at a given point in time $t_{1}$ can be compared to the deviations observed at another point in time $t_{2}$ to confirm a sensor's stationarity. Ideally, the measurements in these two points are the same, but in practice this is not true for all sensors. Metrological properties of sensors are the characteristics that represents how well a sensor operates and are expressed as an average and standard deviation [53].

The authors in [43] compared different commercially available PSM technologies, where metrological properties such as rise-time, total creep, and average drift were computed using long term experiments, and the coefficient of variation was computed using short-term experiments. These metrological properties were quantified with the goal of comparing how well these devices perform in clinical settings, knowing that a patient can lay atop of a mattress for long periods of time, but the patient may also get on and off the bed multiple times per day. Because of this, it is important to know what the behaviour of the sensor is over time, since the values acquired from 
the sensor may have big differences from the moment in which the patient lays on the mattress and seven hours from that moment. At the same time, it is important to know if the sensor has the same behaviour every time that the patient exits and re-enters the bed.

According to [53], repeatability is defined as the closeness of agreement among consecutive measurements for the same measurand performed over short periods of time, under the same operating conditions and using the same measuring instruments. Thus, a perfect instrument is the one that provides the exact measurement over repeated times when the same pressure is applied. However, variability is typically observed in such experiments, even when the same pressure is applied at the same experiment conditions. The variability of the measurements quantifies the degree of agreement between repeated measurements. The smaller the variability around the mean, the more reliable an instrument is across distinct measurements. Repeatability has been represented as the coefficient of variation $(\mathrm{CoV})$ expressed by equation 1 , where the $\mathrm{CoV}$ is expressed as a percentile of variation over repeated experiments.

$$
\operatorname{CoV}(\%)=\frac{\text { Standard deviation }}{\text { Mean }} * 100
$$

The authors in [43] measured the pressure by laying an adult and a neonatal model atop the PSM for a period of one minute, with intervals of one, two and five minutes between each measurement. The mean applied pressure was computed for each of the four measurement periods from which the standard deviation and the mean were computed and used in equation 1, providing the $\mathrm{CoV}$ for the different technologies.

As mentioned before, long-term experiments can characterize the metrological properties of a sensor over extended periods of use. One of the long term metrological properties is the rise time, which is mainly intended to provide information about how quickly a sensor goes from $10 \%$ to $90 \%$ of the steady state value. The faster the response, the greater is the ability of a sensor to 
provide information about higher frequency changes in the measured signals. A longer rise time, on the other hand, indicates that a sensor takes longer to reach the steady state values, which reflects the ability of the sensor to provide faster changes in the measured signal. The steady state of a signal means that the sensor stabilizes at a given value and minimal changes occurs after that period of time when a constant input is applied. However, some sensors continue to increase the measured signals over time due, for example, to systematic creep (see below). Because of this, the authors in [43] considered that the steady state for all sensors were reached at thirty minutes, and the rise time was computed using this value as reference for the 10 and $90 \%$ values.

The metrological property known as creep measures systematic variations in the total pressure over long periods of time. The total creep is computed using the pressure measured at thirty minutes and the pressure measured at the end of the session, divided by the average pressure between these two periods, as seen in equation 2. The authors in [43] used as reference points the times at thirty minutes and 14.5 hours, which provided a percentile change in pressure for these two points in time. This can also be done considering shorter periods of time, which provides an estimate of the creep over shorter fixed window sizes. For example, using a window size of one hour, it is possible to estimate the creep that occurs in this period of time.

$$
\Delta C(\%)=\frac{P_{2}-P_{1}}{\operatorname{Mean}\left(P_{12}\right)}
$$

Another important metrological property is the drift. The drift is often used in the literature to evaluate the variations of the signal over longer periods of time. However, as performed in [43], creep is used to quantify the long term changes in pressure, whereas the drift represents the changes around shorted periods of time. With these two metrics, both long- and short-term changes on the measurements can be evaluated. When reading the values from the sensor over time, there is 
variations around the true value, either due to noise, interference or system characteristics. Ideally, there would not be any variation around the true value. This means that the readings from an ideal sensor would have a standard deviation of zero around the true value. The drift is computed by dividing the standard deviation of the time sampled signals by the mean of these signals over a short period of time and multiplied by one hundred, which results in a percentage change around the mean of a given window. For example, the drift can be computed using a window size of one minute, which will reflect the variations around the mean without being confounded by signal creep, where the mean can drastically change over longer window sizes.

\subsection{Conclusions}

This chapter discussed the existing technologies around pressure sensors and its applications in fields as patient monitoring, robotics, and others. In this thesis, the main focus is to design a pressure sensor to be used in patient monitoring. Later in Chapter 5, we demonstrate the applicability of this sensor for monitoring the breathing rate of a patient laying on top of the prototype, which indicates the suitability of this technology for patient monitoring. Current and proposed technologies to measure force were presented and discussed, providing an overview of the existing product in the market and the challenges with the development of a new technology. Lastly, a brief introduction to the metrological properties is presented, which will be the base for the analysis of sensor properties discussed in chapter 4.

Algorithms for patient monitoring have been widely demonstrated in research settings using existing pressure sensors. However, the cost of these sensors limit deployment of such systems in clinical environments beyond research purposes. Moreover, the existing technologies have strengths and weaknesses points in terms of metrological properties. Altogether, there is space for 
the development of new technologies with improved metrological properties, reduced cost, and ease of manufacturing. 


\section{Design of a novel pressure sensor}

\subsection{Introduction}

This chapter describes the development of the prototype device and partial results regarding the linearity of the measured pressure. The design includes the printed circuit board and the main circuits involved, the silicone rubber layer design and its characterization, and the graphical user interface implemented for signal visualization, and digital communication protocols with the printed circuit board.

\subsection{Design Overview}

\subsubsection{Goals and requirements}

Pressure sensitive mats are advantageous for the measurement of physiological signals in that they are unobtrusive and there is no need to attach sensors to a patient's body. Instead, changes in pressure distribution are measured and analyzed to derive the vital signs. One complication with using PSM in hospital settings is that beds typically comprise distinct structural panels for the head, torso, and legs, and that these panels are movable such that the bed configuration can be changed. The size and shape of these panels vary by bed manufacturer and some bed frames have irregular surfaces. Therefore, a PSM technology should be reconfigurable, flexible, and durable sensing devices to permit widespread clinical deployment. Due to the variety of bed sizes and formats, the sensing technology should be easy to design and adapt accordingly to the needs of specific supports including non-uniform surfaces.

The range of weight for patients varies from less than $0.28 \mathrm{psi}$ for infants to more than 0.35 psi for adults [43]. The ideal sensing device should have a suitable operating range of measurement 
such that the same device is applicable for the majority of hospital's patients. In addition to the mechanical characteristics, it is important to consider the data acquisition properties. Heart rate (HR) and respiration rate (RR) are two vital signs of importance that can be acquired with the use of pressure sensitive mats. HR varies from approximately 30 to 220 beats per minute (bpm), while RR typically falls in the range of 12-20 breaths per minute for normal breathing, whereas abnormal ranges are under 12 and over 25 breaths per minute. Considering these frequency ranges and the Nyquist-Shannon law, a minimum sample rate of $8 \mathrm{~Hz}$ for $\mathrm{HR}$ and $1 \mathrm{~Hz}$ for RR are required. However, a significantly higher sampling rate is beneficial in practice.

\subsubsection{Novel pressure sensor concept}

The main concept of the proposed design is similar to the concept used by Kinoplex sensors, in that the intensity of transmitted light is used as a means to measure pressure. In the case of fiber optics, the light is transmitted from one end of the fiber, affected by a medium such as foam, transmitted back from another fiber, and then measured at the end of the other optical fiber. Similar to the Kinoplex sensors, in the present design, each sensel has an individual LED and a phototransistor. However, instead of changing the reflected light by the compression of a material such as foam, the proposed sensor measures the distance from the LED to the phototransistor within a compressible chamber, similar to [4]. Here, the medium is air, and the top (ceiling) of the cavity is more reflective than the walls of the dome. Different materials and colors were tested in different stages of the project. The silicone rubber rigidity was selected to work in the range of pressures applied by both infants and adults. The color of the silicone rubber was determined through experiments. Bright colors tends to reflect more light than darker colors, therefore different colours were used for the top and walls of the chamber. 
Figure 12 illustrates the concept of the proposed pressure sensitive cell. On the left side, there is no pressure applied to the dome, whereas in the right side, force is applied to the top of the cavity, resulting on the deformation of the dome. This deformation decreases the distance from the integrated circuit to the top of the cavity, causing more reflected light to reach the detector and the measured signal to be increased. The deformation of the internal dome is proportional to the force applied to the top of the cavity. Thus, it is possible to relate the force/pressure applied with the relation between the intensity of light transmitted from the LED and received at the phototransistor.
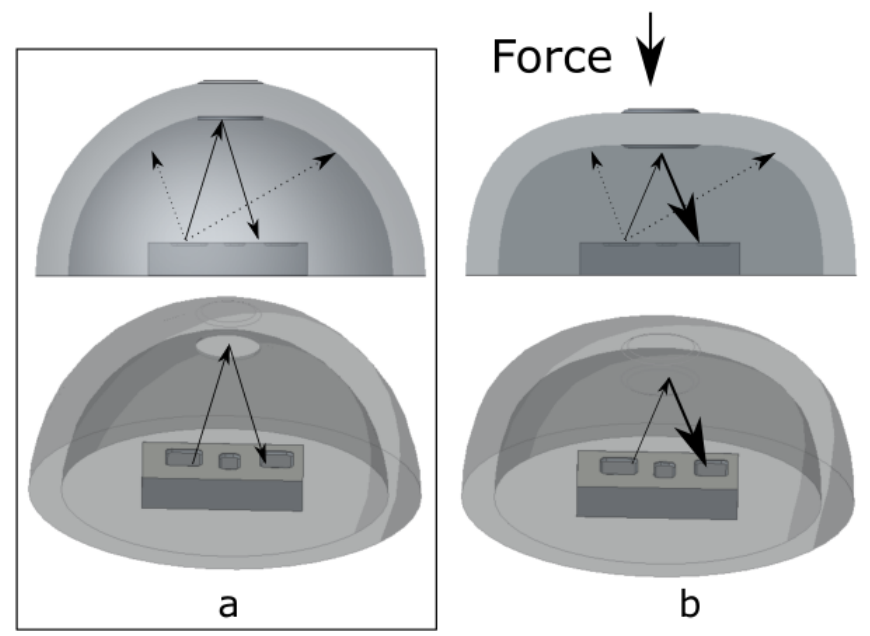

Figure 12 - Concept of pressure sensitive cell. a) No pressure is applied, and b) pressure is applied.

\subsubsection{Design tools}

This section details the design tools employed to develop the prototype PSM in this thesis; each individual component is described in greater detail in subsequent sections. For the development of this prototype, various tools have been used to design, simulate, and manufacture the different parts of the project. The prototype comprises a printed circuit board (PCB), and a silicone layer that lays atop of the PCB. The PCB was designed using the software Eagle, where the manufacture files were generated and sent to PCB Universe to be fabricated. The components used in the PCB 
were soldered by NPS Labs located in Ottawa, Ontario. Creo Parametric computer-aided design (CAD) software was used to simulate the deformation of different types of silicone rubber, as well as to design the molds used to make the silicone layer. The molds designed with the CAD tool were 3D printed using the Cura software and the Ultimaker 2 extended 3D printer. The silicone was then poured into the molds in color order, from the bottom layer to the top. The final silicone layer design used two distinct colors, which are black and white. First the black silicone was poured into the mold to form the walls of the cavity. Then the white silicone was poured on top of the black color after the black color was dry. The white color was used for maximum reflectivity, and the white silicone layer forms the top of the cavity, thereby maximizing the intensity of light reflected back to the sensor.

For data visualization and data acquisition, two software programs have been developed. The first GUI was developed using Matlab and was written with its native language. The second program was developed using Visual Studio and written in C\#. This software sends and receives data throughout the USB connection, configures the microcontroller, provides different layouts, graph plots in real time, and stores the recorded signals to a local computer. The user is able to visualize the signals of each sensor over a short period of time, the sum of all sensors over the entire recording session, or an image with the instantaneous pressure profile with adjustable pressure range, allowing the user to adjust the mapping of measured pressure to colors using a sliding bar for better visualization of the pressure distribution.

After the data are stored on the computer, Matlab is used to process the data, as well as to provide the visualization of the recorded sessions. The metrological properties and the characterization of the linearity of the developed sensor were computed using Matlab. 


\subsubsection{Design of Proximity-based Pressure Sensitive Mat}

The design of the pressure sensitive mat can be divided in two parts: the electronic module, and the top silicone rubber layer. The electronic module prototype comprises a custom flexible printed circuit board. For the purpose of developing a proof-of-concept prototype, this PCB is controlled by a Tiva ${ }^{\mathrm{TM}}$ Board Launchpad TM4C123G, which includes an ARM ${ }^{\circledR}$ Cortex ${ }^{\circledR}$ M4F based microprocessor. Figure 13 shows the block diagram of the implemented system while Figure 14 is a photograph of the prototype. The flexible printed circuit board is populated with an array of VCNL4020 proximity sensors (represented on Figure 13 as "Proximity sensor array"). The VCNL4020, manufactured by Vishay Semiconductors (www.vishay.com), is a fully integrated proximity and ambient light sensor, with 16 bits of resolution and fully configurable through an I2C communication interface. All sensors share the same I2C address. Therefore, a multiplexer block is required to switch the serial data line (SDA) to the desired sensor. The serial clock line (SCL) is shared by all sensors.

The Launchpad board is connected to a computer via USB, and custom software data acquisition was implemented for this prototype. When the user begins a new measurement from the computer, data is transferred via a COM port through USB connection. The user can save the data, and visualize time series of the pressure of each individual sensor and/or the total pressure, or a color image where pixels representing the instantaneous measured pressure. 


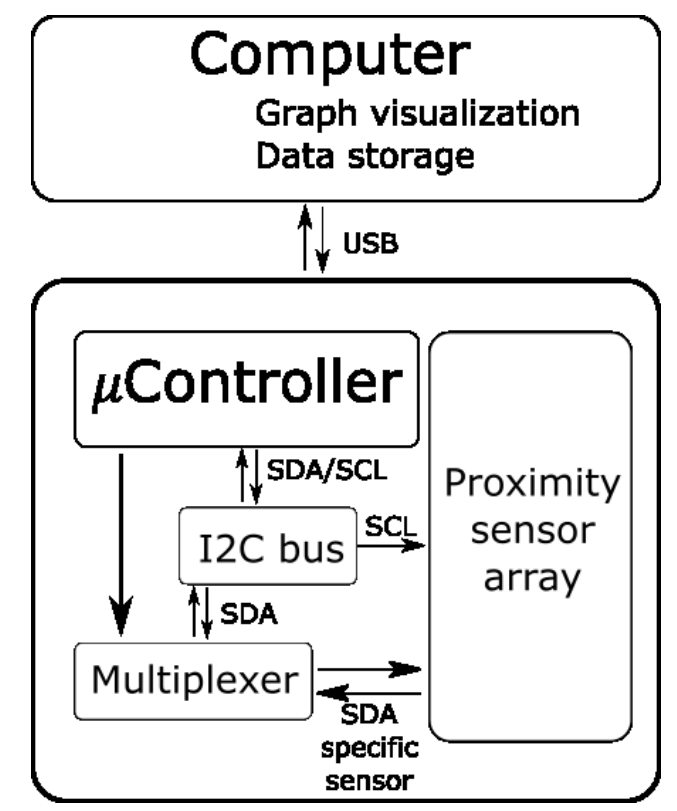

Figure 13 - Block diagram of the implemented system

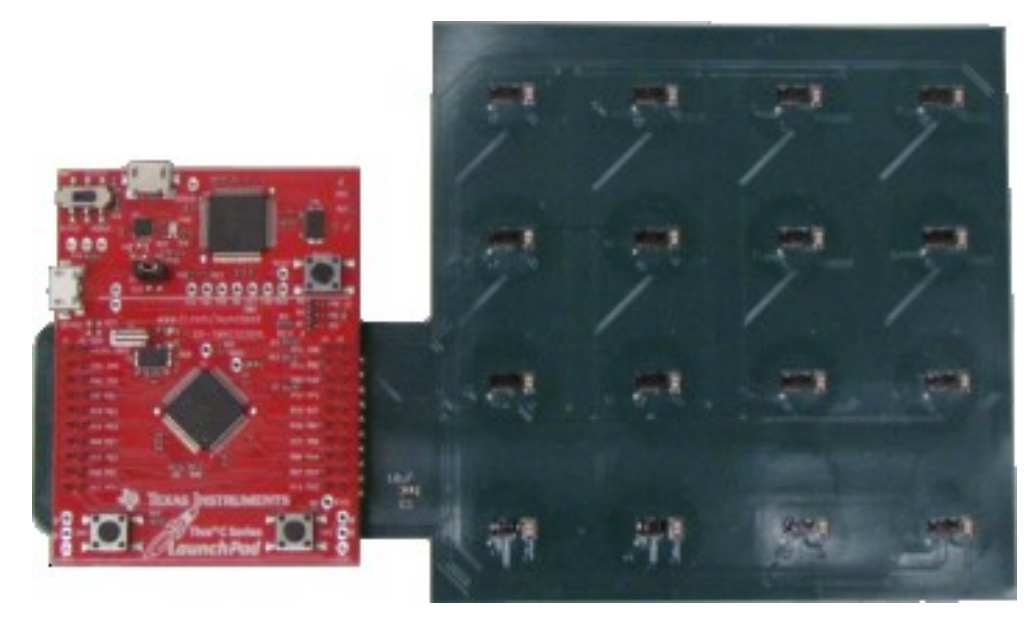

Figure 14 - Flexible printed circuit board with a TivaTM Board Launchpad TM4C123G attached to it. The black rectangles on the green circuit board are the array of proximity sensors.

The flexible printed circuit board has been designed in a square configuration for this prototype; however it can be adapted to the needs of specific projects, such as a bed frame or the bottom of a wheelchair, where sensors are not required in an array form, but only where there is 
contact with the subject. Currently, the data acquisition rate is $50 \mathrm{~Hz}$; higher rates are possible but are not required for the purpose of vital sign monitoring.

Figure 15 shows the elastic layer, which is connected to the top of the flexible printed circuit board. The geometric layout of the layer creates an array of "domes" with flexible bracing between each dome. As pressure is applied on top of the silicone layer, the distance between the top of the dome and the sensor decreases, causing the signal intensities measured by the sensor to increase. The signal measured can then be translated to applied pressure.

The elastic layer is made of silicone rubber Ecoflex ${ }^{\circledR}$ 00-50 manufactured by Smooth-on (www.smooth-on.com). The walls of the dome are made of black silicone rubber, whereas the top of the elastic layer is made of white silicone. Darker colors will absorb the light, whereas lighter colors will reflect light with higher intensity. The use of dark silicone in the walls and white silicone on the top of the elastic layer results in most of the received signal being from direct reflection from the cavity ceiling. Therefore, the intensity of received light is a function of displacement of the top of the dome, and not from the deformation of the walls. Furthermore, the radiation pattern of the VCNL4020 sensor is stronger in the direction perpendicular to the sensor, which means that most of the power irradiated by the emitter will be directed to the ceiling of the cavity. The embedded proximity sensor is also most sensitive to signals incident in a perpendicular direction; the signals from higher angles are attenuated. Appendix A shows the radiating and receiving patterns of the emitter and proximity sensor, respectively. Thus, the signals measured by the proximity sensor will be mainly from the top cavity for three reasons: due to the colouring of the silicon, the walls of the cavity attenuate the signals while the ceiling amplifies the reflected signals; the power of the emitted light is focused on the ceiling instead of higher angles; and the receiver minimizes the signals from higher angles while maximises the perpendicular angles. 


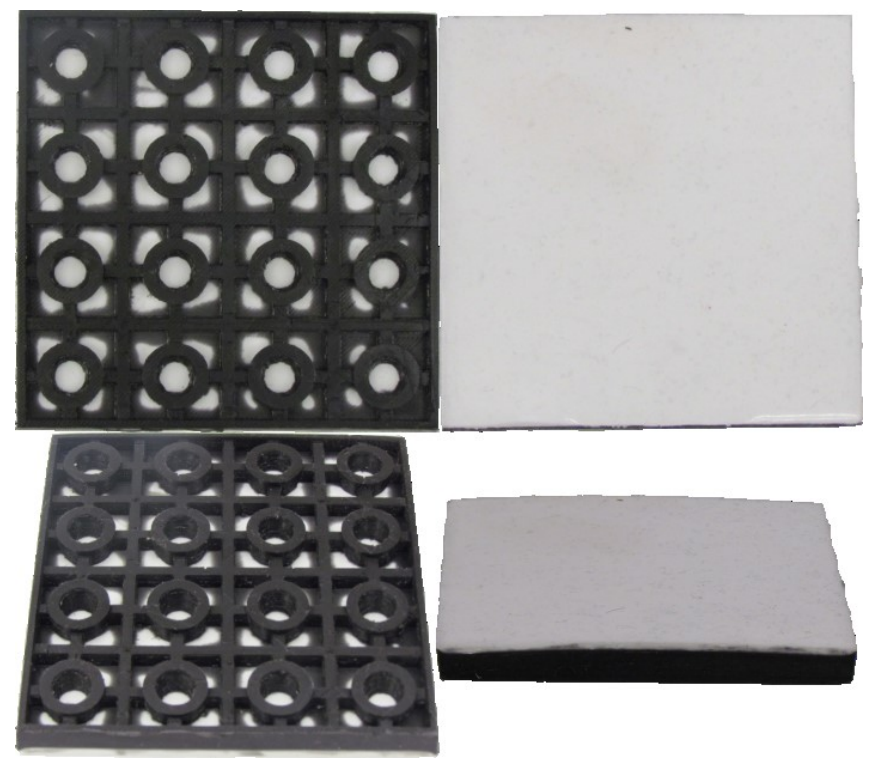

Figure 15 - Bottom, top, and front views of the silicone elastic layer.

\subsubsection{Signal conditioning}

The proximity sensors measure the distance from the sensor to the top of the silicone cavity by emitting pulsed infrared light and measuring the reflected light from the cavity. A baseline signal is measured when there is no pressure applied to the device. For each experiment, a one minute unloaded baseline recording was first made. For sensor $i$, the pressure baseline, $P B_{i}$, is computed as the mean of these data. By subtracting $P B_{i}$ from the acquired data, the zero level corresponds to be the no-pressure state.

The sensor pressure (SP) is expressed by equation 3, where the actual measurement at time n, $s_{(i)}(n)$, is shifted by the pressure baseline. Here, the measurement is in counts, denoted as cts, representing the raw value read by the sensor with sixteen bits of resolution. The total pressure (TP) is the sum of all sensors as shown in equation 4.

$$
S P_{i}(n)=\left(s_{i(n)}-P B_{i}\right)[c t s]
$$




$$
T P(n)=\sum_{i=1}^{16} S P_{i(n)[c t s]}
$$

To test for the linearity of the sensor, different weights were applied on top of the silicone layer, with a hard and flat circular surface underneath the weight with an area of $10.53 \mathrm{in}^{2}$. In order to calibrate the sensor, a $100 \mathrm{~g}$ weight was placed on the sensor for one minute. After the total pressure was found, the mean of $T P_{100_{g}}(n)$ was computed for $n=5$ to 55 seconds (i.e. equation 5 with $\mathrm{n}_{1}=5$ and $\mathrm{n}_{2}=55$ ) and normalized by the area of the circular surface explained above. Equation 6 defines the normalized total pressure (NTP), which is then used to measure applied pressure in subsequent experiments using units of pounds per square inch (psi). This equation assumes that the intensity of received light is linear with respect to applied pressure; this assumption is confirmed later in this chapter.

$$
\begin{gathered}
T P_{100_{g}}=\frac{1}{n_{2}-n_{1}} \sum_{n=n 1}^{n 2} T P(n)[c t s] \\
N T P(n)=\frac{T P(N)}{T P_{100_{g}}} * \frac{0.1 k g}{0.453592\left(\frac{k g}{l b}\right)} * \frac{1}{10.53 i^{2}} \\
N T P(n)=\frac{T P(n)}{T P_{100_{g}}} 0.020937[p s i]
\end{gathered}
$$

\subsubsection{Graphical User Interface}

Two GUI were implemented in this project, in which signals are acquired in real time and sensor configurations are set through a USB connection. The USB connection emulates a COM Port, which is a serial protocol. The user must select the COM port configured on the computer to have 
access to the PCB data. Figure 16 shows the configuration tab where the COM port is selected. It is possible to set different electrical current values on the VCNL4020 sensor, ranging from 10 to $200 \mathrm{~mA}$ of modulated current which impacts the intensity of emitted light within the chamber. The current is set using the drop down menu of the "Current" field on the configuration tab. It is also possible to set the sample rate at which data is collected. The available sample rates are 50, 25, 10, $5,1,0.5,0.25,0.125$ and 0.01 samples per second. The user can also select the folder in which the files will be stored, and set the name of the session being recorded. If the field "File name" is left blank, no file will be stored. When the field is not left blank, a file will be written to the specified path, where time-stamped samples are appended as received.

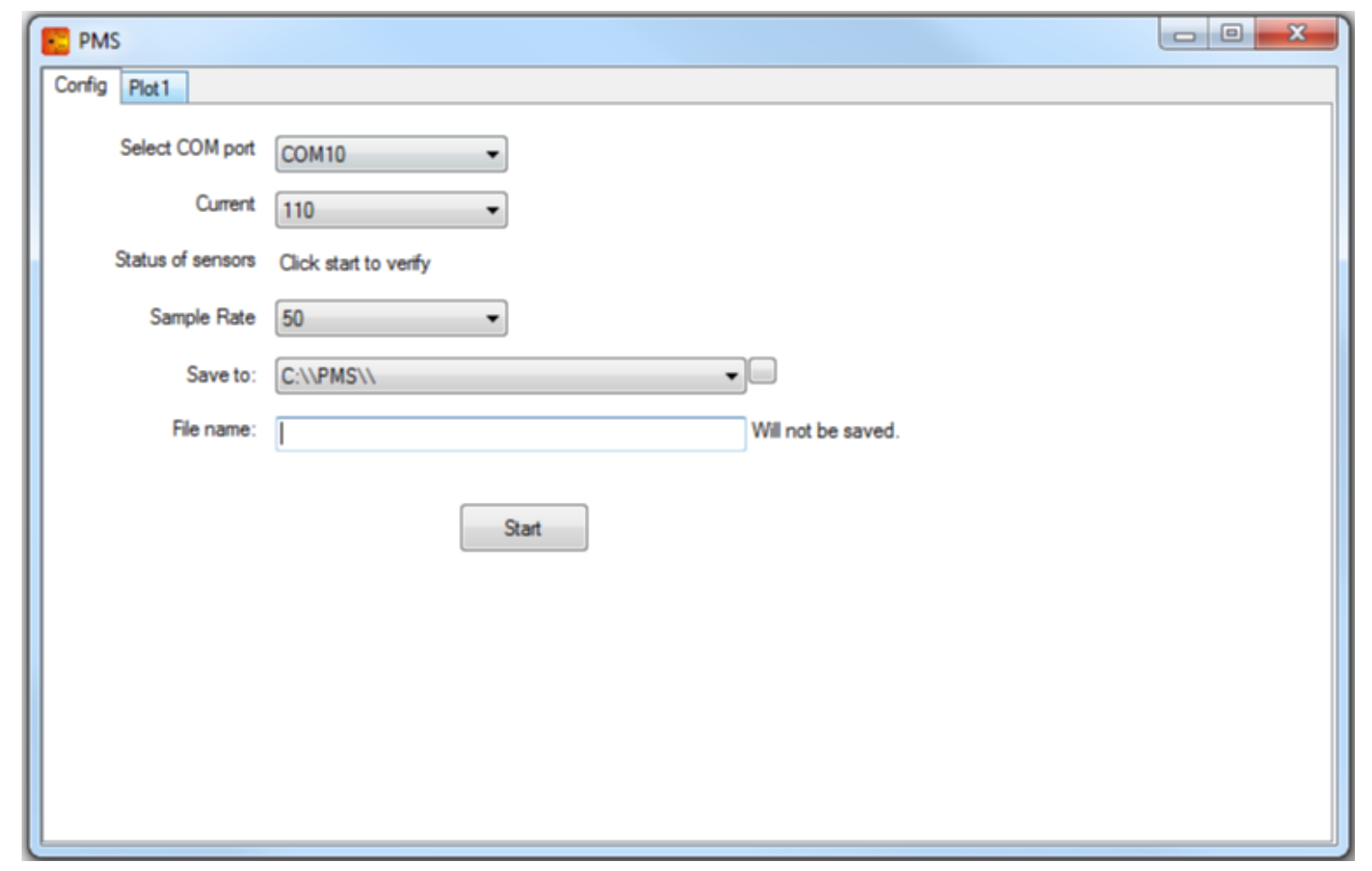

Figure 16 - Main tab of the GUI developed to communicate with the prototype to set configurations and receive data.

Once the user starts a new session by clicking at the "Start" button, the "Plot1" tab is activated, and the measured signals are visualized in real time. Each sensor is plotted on the tab 
"Multiple Sensors", where each individual sensel have its own line on the scrolling time series plot. The length duration of the visualized signal is 20 seconds, where the new samples are appended to the line. When the signal overflows the 20 seconds window, all lines are shifted 3 seconds to the left. The update rate of the lines is the same as the sample rate set by the user at the configuration tab. Figure 17 shows an example of the multiple sensor visualization, where each sensor is represented by one line.

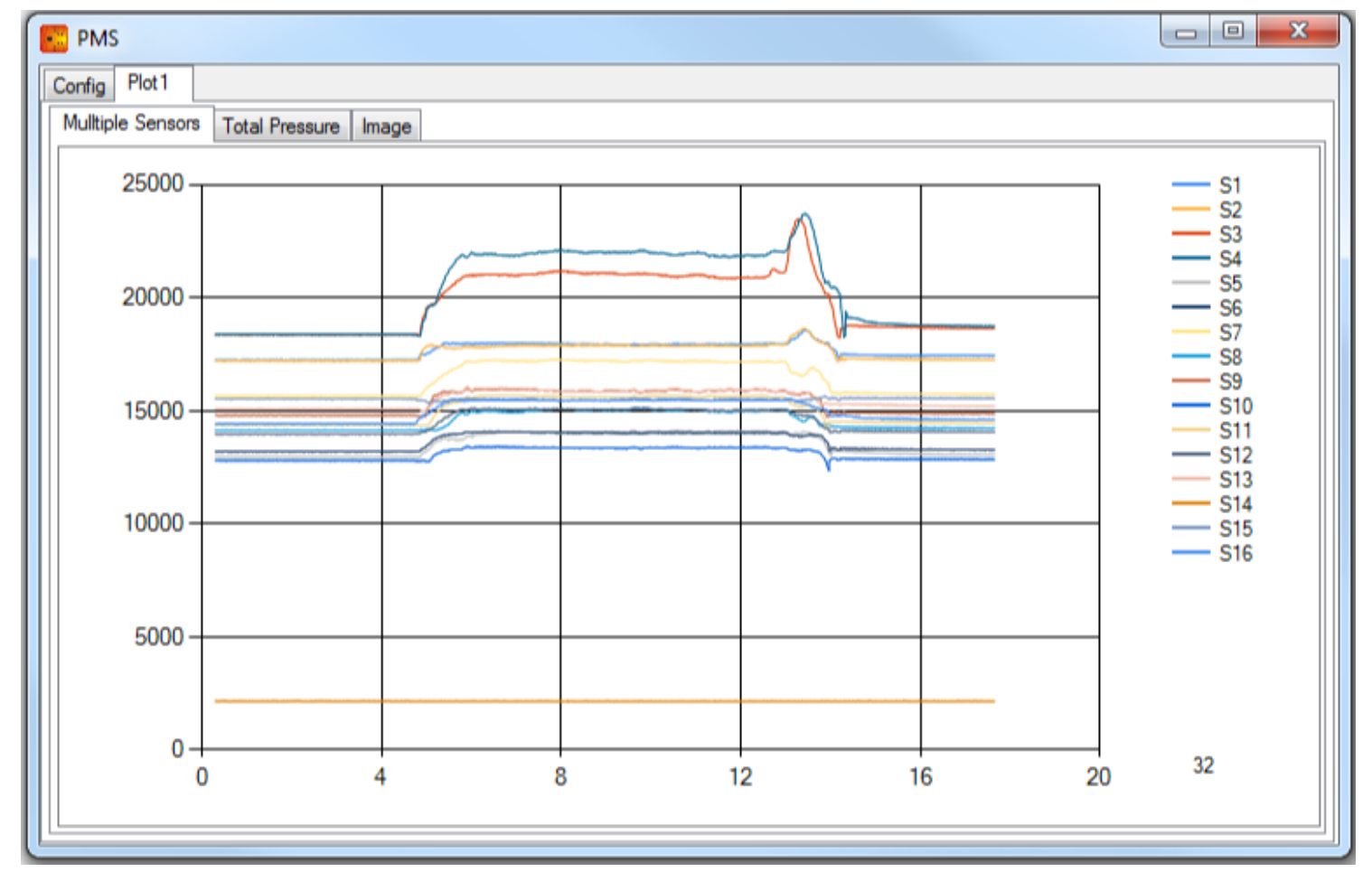

Figure 17 - Multiple Sensors tab of the implemented GUI. Each line represents the values read for each sensor.

The "Total Pressure" tab shows the total pressure applied over time (equation 4). It is the sum of all values read from each sensor in each sample. Figure 18 shows an example of the total pressure tab, where the line represents the sum of all sensor values over time. Figure 19 shows the "Image" tab, where each image is created from the last sensor values read. For visualization purposes only, interpolation is used to increase the number of pixels in the image. This makes the 
image look smoother instead of comprising only 16 discrete squares, each one representing the measurements of one of the sensors from the prototype. The slider positioned below the image allows the user to adjust the color map of the image, easily adapting the range of colors based on the upper saturation point set by the user.

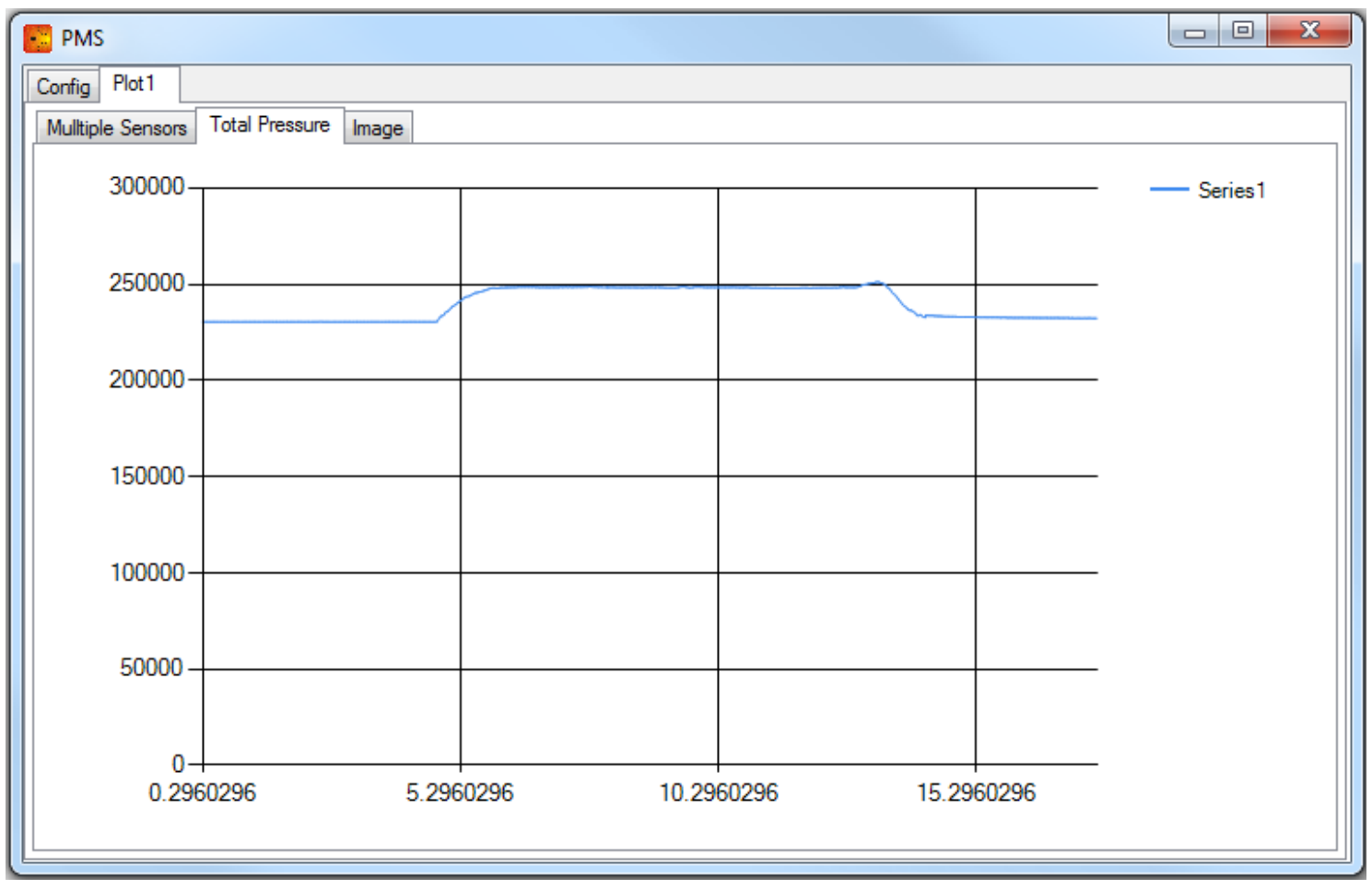

Figure 18 - Total Pressure tab of the implemented GUI. Each point in the line represents the sum of the values read from all the sensors. 




Figure 19 - Image tab of the implemented GUI. The values from the sensors are interpolated to generate an image based on the pressure applied to each individual cavity. The color map is adjusted by the user in the slider below the image.

\subsection{Results}

\subsubsection{Simulation results}

Finite element analysis was used to design the geometry of the silicone rubber layer. We require that the entire PSM be flexible and that the cavity above each sensor deform linearly with applied pressure. Therefore, the silicone rubber layer was designed to have individual cavities with a roughly dome shape, with minimal connecting support structure between each cavity to maintain overall flexibility. The overall design of the silicone layer is shown in Figure 15. Finite element analysis was conducted using Creo Parametric simulations tool. An elastic material was assigned to the elastic layer, and simulations were performed for a range of pressures applied on top of the layer. Figure 20 shows a FEA simulation performed for pressures of $0.1,0.2,0.3$ and 0.4 psi. It 
can be seen that the displacement of the cavity is proportional to the force applied to the top of the layer, and that as the pressure increases there is an increase of displacement in the y axes.

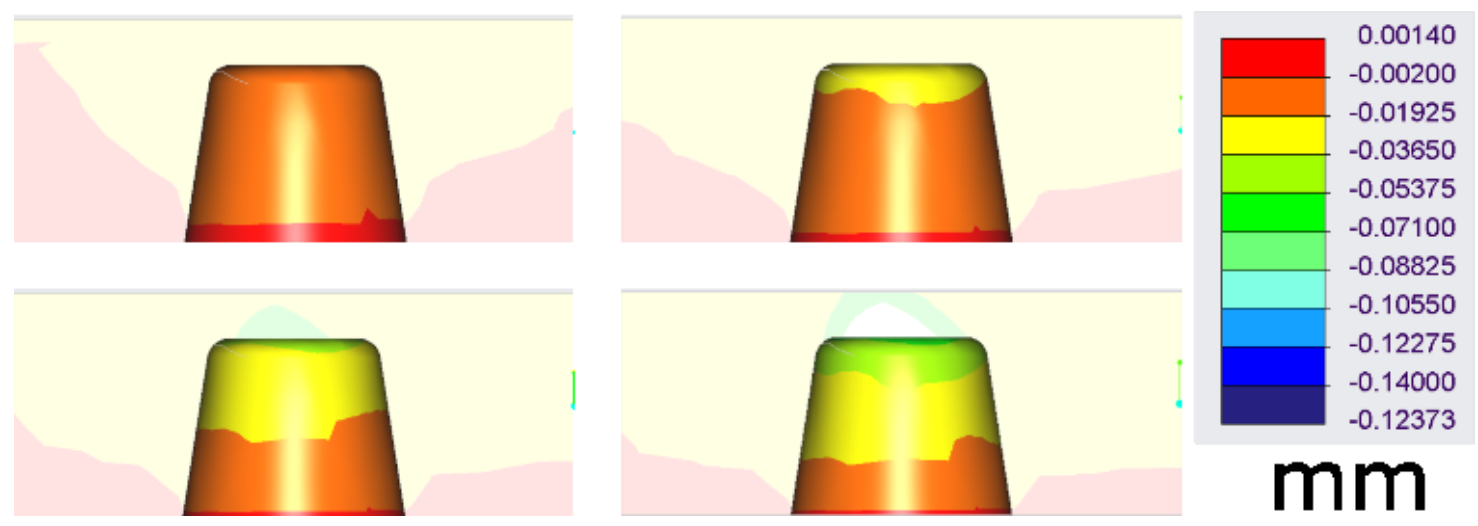

Figure 20 - FEA simulation for 0.1 (top left), 0.2 (top right), 0.3 (bottom left) and 0.4 (bottom right) psi applied in the Yaxis. Colouring represents the magnitude of displacement.

The FEA simulation provided information about the deformation of the silicone layer. However, the deformation of the silicone rubber is not the only variable that changes the intensity of light reflected from the cavity. In fact, the colors of the silicone rubber have significnat impact on the reflected light. Multiple colors were explored in this study. As expected, it was found that darker colors of silicone rubber attenuate the measured signal in higher degrees than lighter colors. The final configuration of the silicone rubber resulted in black colored walls with a white ceiling. This configuration and the fact that the photo emitter and detect on the VCNL4020 are highly directional, results in most detected light being reflected from the cavity ceiling and not the walls.

\subsubsection{Experimental results}

To test the assumed linear behaviour of the prototype, weights were applied on top of the prototype, remaining there for a period of one minute, and then removed from the top of the cavity for another minute. The weights varied from $100 \mathrm{~g}$ to $1700 \mathrm{~g}$, incrementing by $100 \mathrm{~g}$ every cycle. The weights 
were applied on top of a circular and hard flat surface with radius of $4.65 \mathrm{~cm}$, which was used to compute the pressure applied per unit area. The measurements were then normalized using equation 6, where $T P_{100_{g}}$ was computed using the $T P$ from one to two minutes of the recorded signal, which is the segment where $100 \mathrm{~g}$ was applied to the cavity, and $P B_{i}$ was computed using the first 55 seconds of the recorded signal where no weight was applied other than the circular disc. For each weight, the mean observed pressure was computed using 50 seconds of data, i.e. from 5 seconds after the pressure was applied and 5 seconds before the pressure was released.

Figure 21.a shows the normalized measured signal, the actual applied pressure, and the mean for each weight application. A first order model was then best-fit to the mean measured pressures vs. applied pressure. Figure 21.b shows the actual vs. measured pressure along with the linear model. Using a polynomial equation of order one, it was possible to reach a R-squared of 0.995 , which shows that the prototype is linear over the range of 0.02 to $0.35 \mathrm{psi}$. This experiment shows that this prototype could be used for both children and adults.

Examining the first-order model in Figure 21, it can be seen that there will be negative values for very small applied loads; this can be easily solved by eliminating any signal below zero. By increasing the order of the polynomial model, the R-squared measure increases and the signals below zero are attenuated, allowing a more precise representation of the pressure applied. However, higher order polynomial equations may lead to unexpected artifacts, especially at the extremes and between measured samples. Even though the extremes were not evaluated in this chapter, a linear model performed sufficiently well in this case and higher order models were deemed unnecessary. 

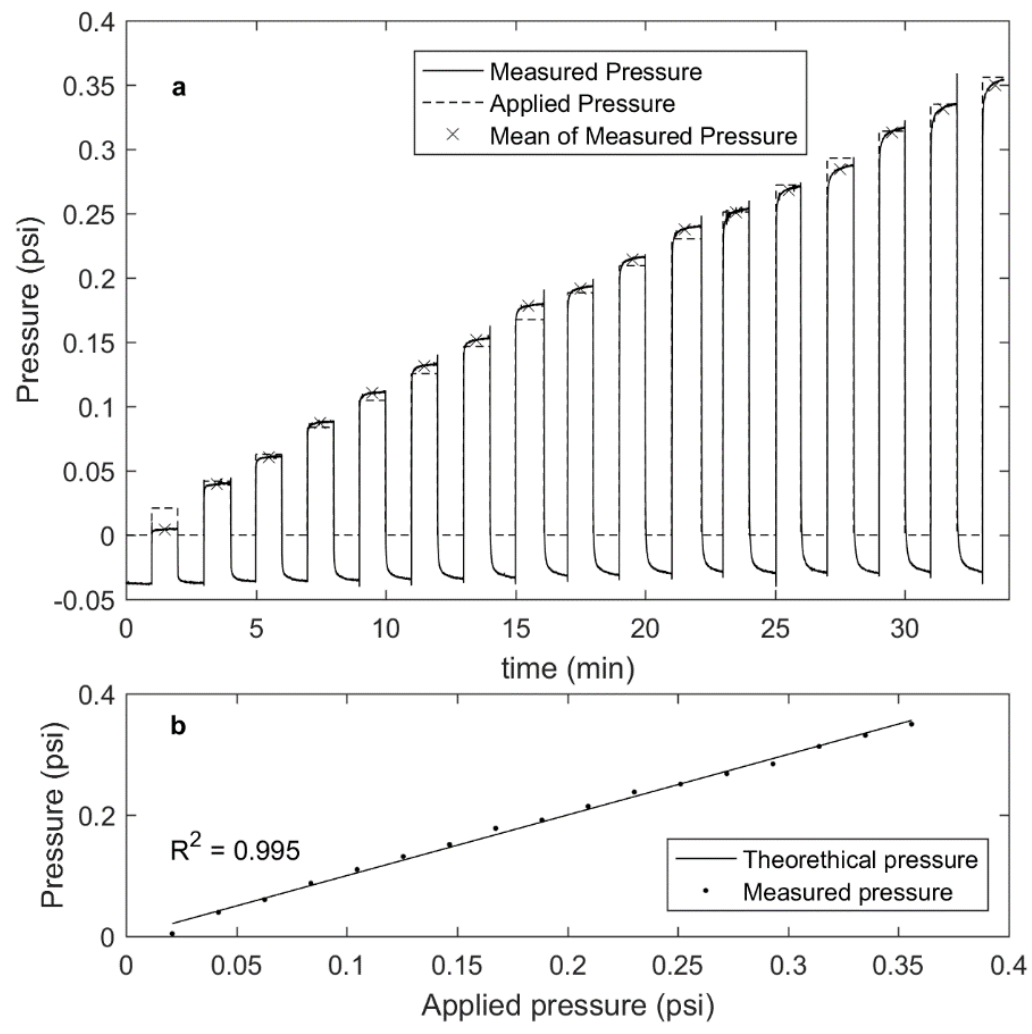

Figure 21 - Experiment of linearity. a) Incremental weights of $100 \mathrm{~g}$ are placed on top of a circular surface over a period of $1 \mathrm{~min}$ and removed from the surface for $1 \mathrm{~min}$. b) graph shows the applied pressure in the $x$-axis and the measured pressure in the $y$-axis.

\subsection{Conclusion}

The prototyped device was designed to be flexible for non-regular surfaces with moving parts such as hospital beds, meeting the requirements established in this chapter. With the employed sensor there is a possibility of designing less expensive pressure sensor mats, with higher adaptability that can be designed and deployed in a variety of clinical environments. The new technology uses proximity sensors to measure the distance from the sensing IC to the top of a cavity made of compressible material. By measuring the intensity of reflected light within the cavity, material displacement is converted to applied pressure. Experimental results show that the prototype 
exhibits a linear response over the range required for monitoring patients ranging from children to average adults. The ease of manufacturing and the price of the components employed on the prototype indicate that this technology could represent a more economical solution for small to large scale production, thereby encouraging deployment of this technology for non obtrusive monitoring in clinical settings. In the following chapter, experiments are conducted to quantify the metrological properties of the novel sensor described in the current chapter. 


\section{Evaluation of Metrological Properties}

\subsection{Introduction}

It is important to know the behavior of a sensor in a given setup, which might include short or long-term data acquisition.

The use of PSMs in long-term monitoring has been exploited in some studies [3], [4]. In such cases, it is important to recognize and evaluate the effect of sensor changes due to long-term monitoring, since the measures may be affected with regards to time. With the metrological properties of a given sensor, researchers and engineers can identify the qualities and limitations of a device, thus being able to draft more precise conclusions from the acquired data. In this chapter, the dynamic metrological properties of the implemented system are analyzed and compared with the S4 (optical) sensor, considering long-term experiments with two different masses on top of the aforementioned devices. A discussion about other technologies is also performed in Section 4.4, considering the findings in [43].

Some of the important metrological properties are: drift - a variation of the sensor measurement unrelated to any stimulus; coefficient of variation - a measure of the dispersion around the mean measurement; rise time - the time a sensor takes to go from $10-90 \%$ of the steady state; and creep - a systematic, often monotonic change in sensor measurement observed over longer periods of time. The definition of these properties were discussed in section 2.3 and will be the focus of this chapter, where short and long term experiments were used evaluate these metrics. 


\subsection{Methods}

Three commercially available pressure sensor technologies and the developed sensor were placed on the floor and weights were added on top of them in short and long term experiments. Each experiment is run for light weight, equivalent to $\sim 1300 \mathrm{~g}$ and heavy weight, equivalent to $\sim 1700 \mathrm{~g}$. In all experiments a metal plate is used as support of the masses added, so that the weight distribution across the plate is captured by all the sensors underneath the plate. Taking into account the area of the metal plate, the corresponding contact pressures for light and heavy weights were 0.28 and 0.37 psi, respectively. For short term experiments, the weight was placed on top of the plate for one minute four times, with unloaded intervals of one, two and five minutes. For long term experiments, the weights were added to the plate and the pressure sensitive mats recorded the signals for periods greater than eleven hours.

The devices used in this experiment are the proposed PSM technology sampled at $20 \mathrm{~Hz}$, the XSensor LX100:100.100.05 (XSensor Technology Corp. Calgary, Canada, XSensor.com) with spatial resolution of $5.08 \mathrm{~mm}$ and sampled at $10 \mathrm{~Hz}$, the S4 PSM (S4 Sensors Inc) embedded with Kinotex fiber-optic sensors (Nitta Corp., Osaka, Japan) with spatial resolution of $\sim 80 \mathrm{~mm}$ and sampled at 17Hz, and a Tekscan BRE5400-1 PSM (Tekscan, Boston, USA, tekscan.com) with spatial resolution of $17 \mathrm{~mm}$, sampled at $12.5 \mathrm{~Hz}$. Figure 22 shows the commercially available mattresses used in this study. 

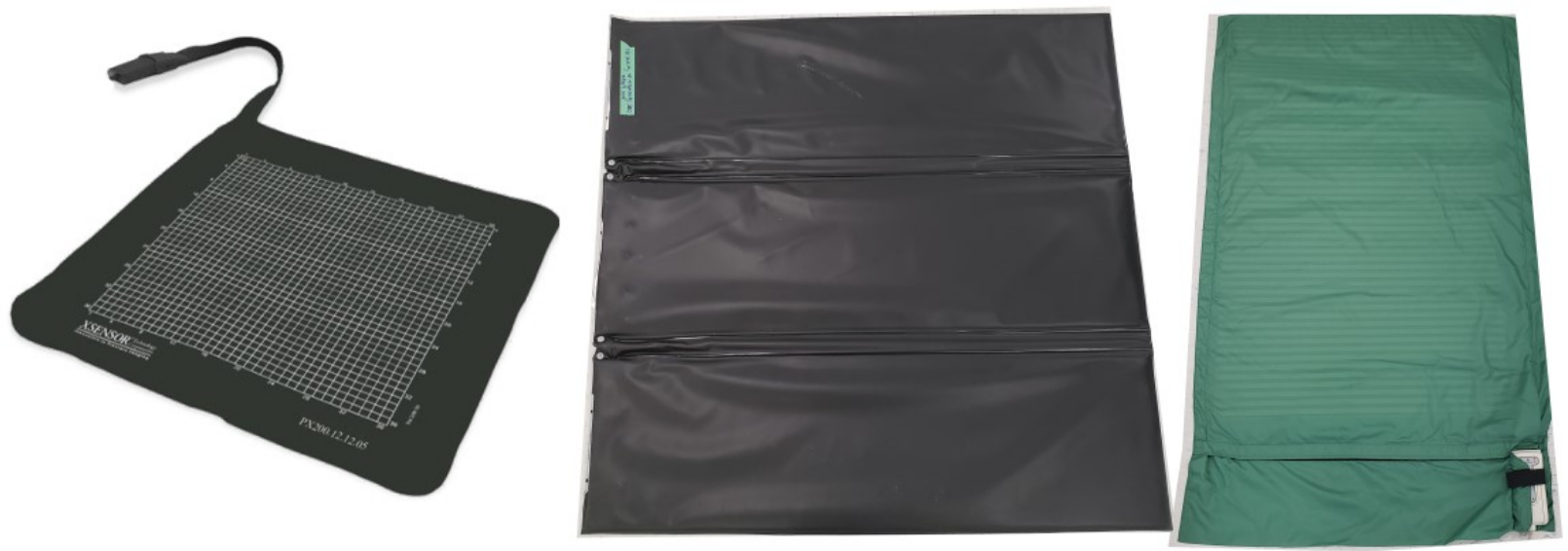

Figure 22 - Commercially available sensors used in this study. Left: XSensor, middle: S4, and right: TekScan

The main goal of these experiments is to characterize the metrological properties of the proposed system and existing technologies in the same experimental scenarios. Based on the collected signals, metrological properties such as rise time, total creep, average drift and coefficient of variation are computed for each technology. With the numerical values obtained through the metrological properties formulas and procedures, and based on empirical analysis, a comparison of the implemented system and existing technologies is conducted.

For long term experiments, the metrological properties of interest are the rise time, total creep, and average drift, which were detailed in section 2.3. The data collected in this experiment is normalized such that the steady state for each sensor reached the true pressure applied ( $0.28 \mathrm{psi}$ for the light weight, and $0.37 \mathrm{psi}$ for the heavy weight). For this purpose, steady state was estimated using the mean over a one minute reference window, approximately thirty minutes from the time at which the weight was added to each technology. For rise time, a moving average filter using a rectangular window of one second is applied to smooth the signal, reducing the characteristic noise form each sensor. The rectangular window moving average is a low pass filter that attenuates the 
effect of noise on the rise-time analysis. Some technologies have considerable noise, which could lead to erroneous shorter rise estimates if filtering is not included. The total creep was computed using, as reference points, one minute windows at the thirty minute and eleven hour marks after application of the weight. Again, the reason to use a window size of one minute is to reduce the effect of noise. The change in creep over time is computed using a window size of one hour, so that it is possible to compare the creep over time for all technologies. The drift is computed starting from thirty minutes until the end of the session, using a window size of one minute. This results in a vector of computed drifts, which are then used to compute the average drift for each technologies.

For short-term experiments, weights representing infants and adults are positioned on top of the sensor surfaces for a period of one minute, and then removed from the surface for a period of time. The light weight $(0.28 \mathrm{psi})$ and heavy weight $(0.37 \mathrm{psi})$ were considered for this study as reference weights, following the same weights applied in [43], where the infant model had a pressure distribution of $0.28 \mathrm{psi}$ and the adult model $0.37 \mathrm{psi}$. This period of time in which there is no weight on top of the surface is one, two, and five minutes respectively. The mean of the interval in which the weight was being applied to the top of the cavity is computed using fifty seconds of data, starting after five seconds from the time in which the weight was positioned on the surface, and finishing fifty seconds after that point. This procedure avoids the transients and also possible mechanical noises related to positioning of the weight. The resulting means from each of the four weight applications are used to compute the mean and standard deviation used in Equation 1 to estimate the $\mathrm{CoV}$. The smaller the $\mathrm{CoV}$, smaller will be the variation around the mean, producing a more repeatable measured signal over different sessions. 


\subsection{Results}

As explained in section 4.2, short and long term experiments were performed using different PSM technologies.

\subsubsection{Short term experiments}

Figure 23 shows the collected data for the short term experiments for the developed technology (labelled here as Dome) and for the XSensor, S4, and Tekscan commercially available PSM. The lines represent the experiments for the light and heavy weights, $\sim 0.28$ psi and $\sim 0.37$ psi respectively. All sensors have been normalized to the aforementioned pressure using the first segment in which weight was applied, and the baseline (estimated over the one minute prior to the first weight application) was set to zero. Four measurements are then used to compute the CoV. It can be seen that for all sensors there is a trend in the measured signal in which the signal increases with time. This appears to be creep, a form of hysteresis, and is further analysed in the long term experiments. It can also be visualized that some sensors have higher variability around the computed mean. The S4 PSM used in this study was particularly old equipment (approximately 10 years), which may explain why it produces elevated noise levels, when compared to the other technologies. This noise is characterized in the long term experiments when quantifying drift.

After computing the means over the intervals, the $\mathrm{CoV}$ was computed using Equation 1. The results of the short term experiments can be seen at the bottom of the Table 1 and Table 2 . The CoV of the proposed PSM technology is the second smallest value when low weight is used on the experiments. The CoV of the PSM is 1.14, whereas for the XSensor, which has the smallest $\mathrm{CoV}$ over all technologies when using the low weight, has a $\mathrm{CoV}$ of 0.99 . The $\mathrm{S} 4$ has a CoV of 1.91 and the Tekscan mattress has a $\mathrm{CoV}$ of 6.27 . Thus, for the low weight analysis, it is possible to conclude that the developed technology is $15 \%$ higher than the best technology, but is $67 \%$ 
smaller than the next-best technology. Figure 24 shows the distributions of the four means computed over fifty second windows for light and heavy weights for the four different technologies used in the short-term experiments. Large vertical bars represent higher $\mathrm{CoV}$, which indicated a higher fluctuation in measured pressure observed over repeated weight applications. Figure 25 shows the same distributions as in Figure 24, but normalized by the mean pressure over the four repetitions such that each technology reports an applied pressure of 1.0, on average. This is done to more easily compare the width of the box plots for each PSM technology.

For the heavy weight, the CoV of the proposed PSM technology (Dome) is the smallest over all other technologies. The computed CoV of the PSM for the heavy weight is 0.8 , followed by XSensor (1.28), S4 (1.8), and Tekscan (7.75). In this case, the second best technology presented a CoV sixty percent higher than the Dome technology. As can be seen, the Tekscan PSM presented the wider distribution for both weights. It can also be seen that the CoV of the Tekscan technology is greater than the $\mathrm{CoV}$ computed for the S4 technology, which does not agree with the results found in [43], where the computed $\mathrm{CoV}$ of the Tekscan equipment was almost half of that experienced in S4 equipment for the neonate (light) model, and less than a third for the adult (heavy) model. These experimental results are further discussed in Section 4.4. 
(a) Dome

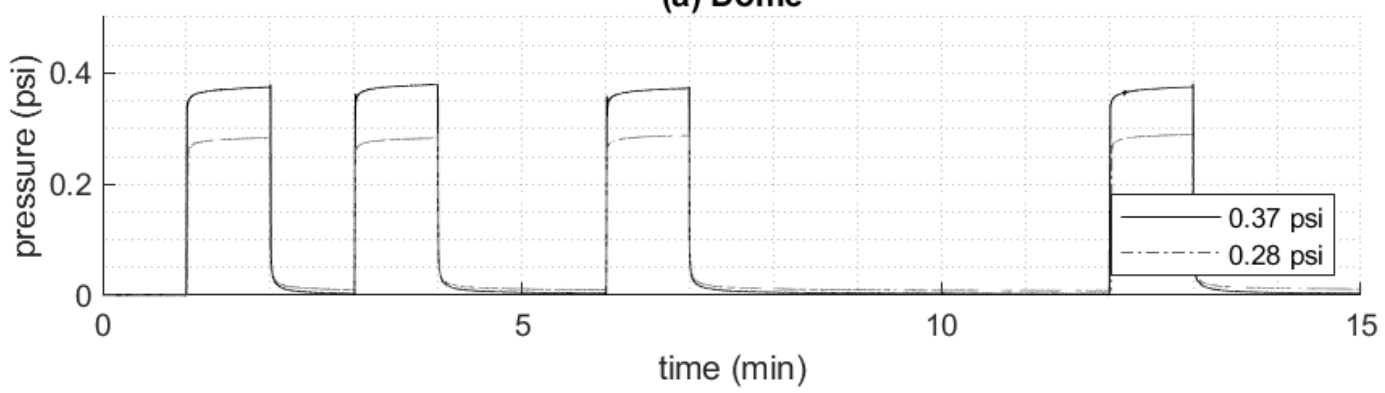

(b) XSensor

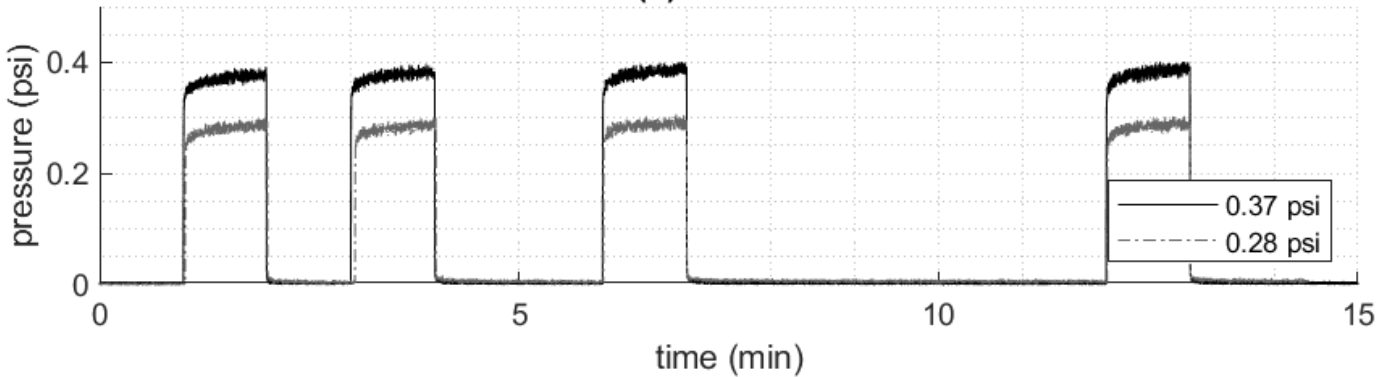

(c) S4

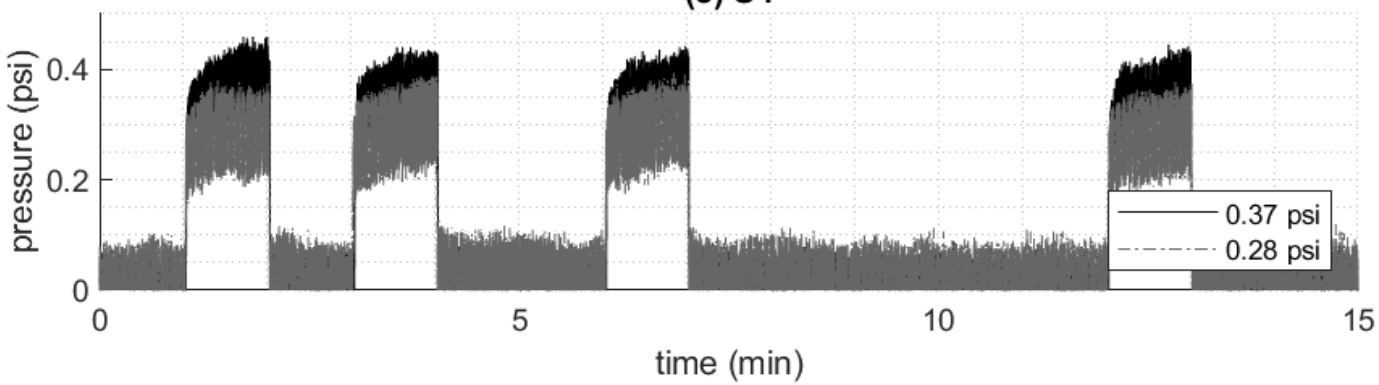

(d) Tekscan

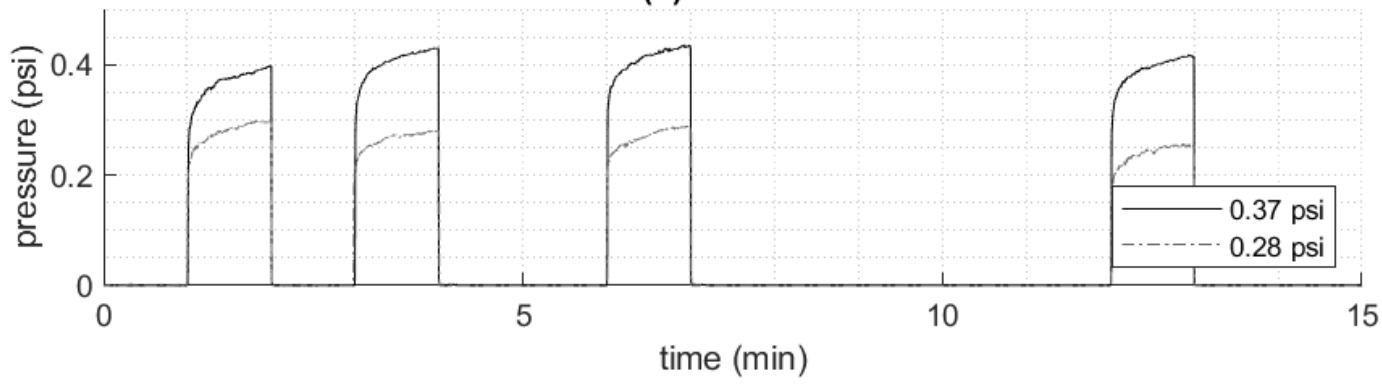

Figure 23 - Short term experiment for four different PSM technologies. Weights are added on top of the PSM four times for one minute each, with unloaded intervals of one, two and three minutes. 
Table 1 - Comparison of metrological properties between the proposed "Dome" system and other commercially available technologies using the light weight

\begin{tabular}{l|c|c|c|c} 
Metrological Property & \multicolumn{4}{c}{ Equipment - 0.28psi } \\
\hline \multicolumn{2}{c|}{ Long-term experiments 11 hours in duration } \\
\hline Applied pressure & Dome & XSensor & S4 & Tekscan \\
\hline Rise Time (sec.) & 1.4033 & 143.3517 & 17.5317 & 24.4682 \\
\hline Total Creep (\%) & 1.6322 & 16.1068 & 8.9479 & 4.3334 \\
\hline Average Drift (\%) & 0.0840 & 4.8955 & 16.1652 & 0.3055 \\
\hline
\end{tabular}

Short-term experiments with 4 repetitions of 1 minute each

\begin{tabular}{c|c|c|c|c}
\hline Applied pressure & Dome & XSensor & S4 & Tekscan \\
\hline $\begin{array}{c}\text { Coefficient of Variation } \\
\text { (Repeatability), CoV (\%) }\end{array}$ & 1.14 & 0.99 & 1.91 & 6.27 \\
\hline
\end{tabular}

Table 2 - Comparison of metrological properties between proposed "Dome" system and other commercially available technologies using the heavy weight

\begin{tabular}{l|r} 
Metrological Property & Equipment $-0.37 p s i$
\end{tabular}

Long-term experiments 11 hours in duration

\begin{tabular}{c|c|c|c|c}
\hline Applied pressure & Dome & XSensor & S4 & Tekscan \\
\hline Rise Time (sec.) & 1.4270 & 143.3772 & 71.4998 & 44.3228 \\
\hline Total Creep (\%) & 1.4670 & 16.1143 & 15.92 & 8.2372 \\
\hline Average Drift (\%) & 0.07 & 4.8960 & 7.6091 & 0.2986 \\
\hline
\end{tabular}

Short-term experiments with 4 repetitions of 1 minute each

\begin{tabular}{c|c|c|c|c}
\hline Applied pressure & Dome & XSensor & S4 & Tekscan \\
\hline $\begin{array}{c}\text { Coefficient of Variation } \\
\text { (Repeatability), CoV (\%) }\end{array}$ & 0.80 & 1.28 & 1.80 & 4.79 \\
\hline
\end{tabular}


(a) light weight

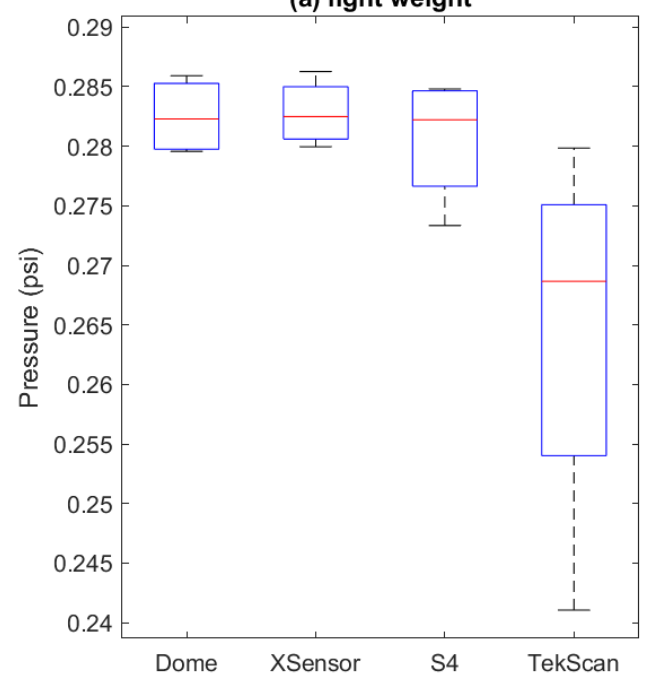

(b) heavy weight

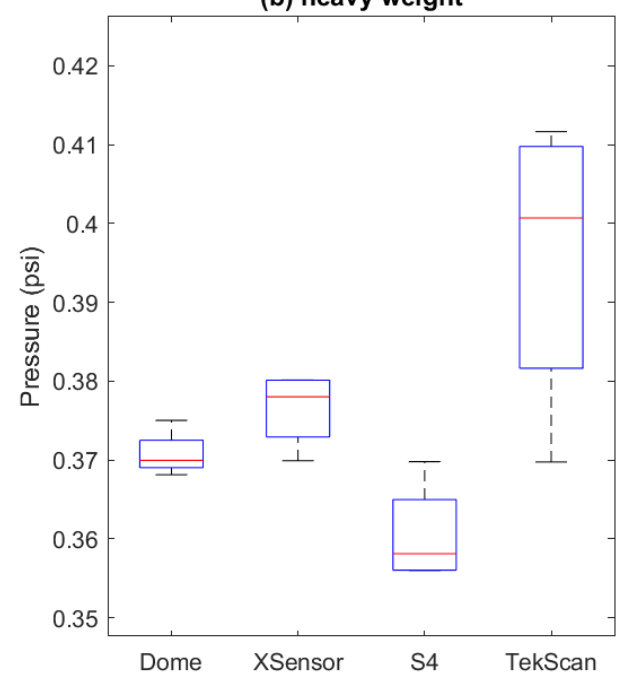

Figure 24 - Average pressure over fifty seconds with weights applied four times between unloaded intervals of one, two and five minutes in the short-term experiment. (a) Light and (b) heavy weight applied to the pressure measurement system.

(a) light weight

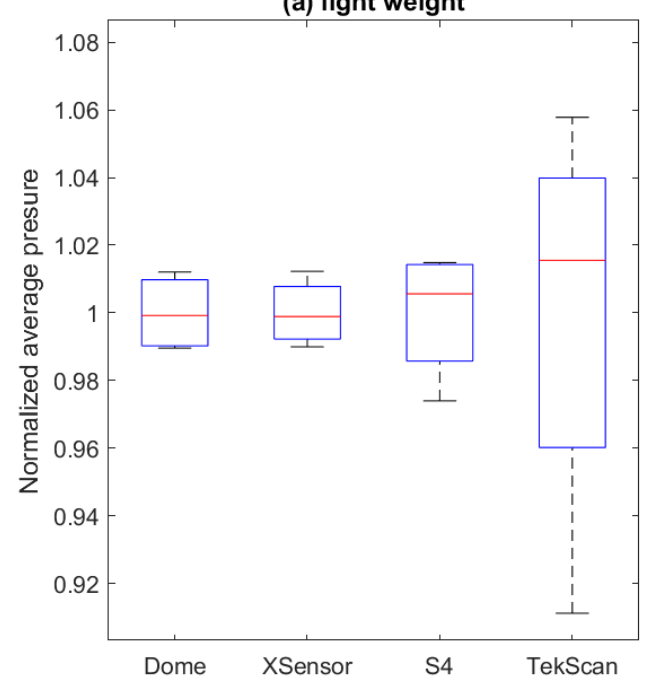

(b) heavy weight

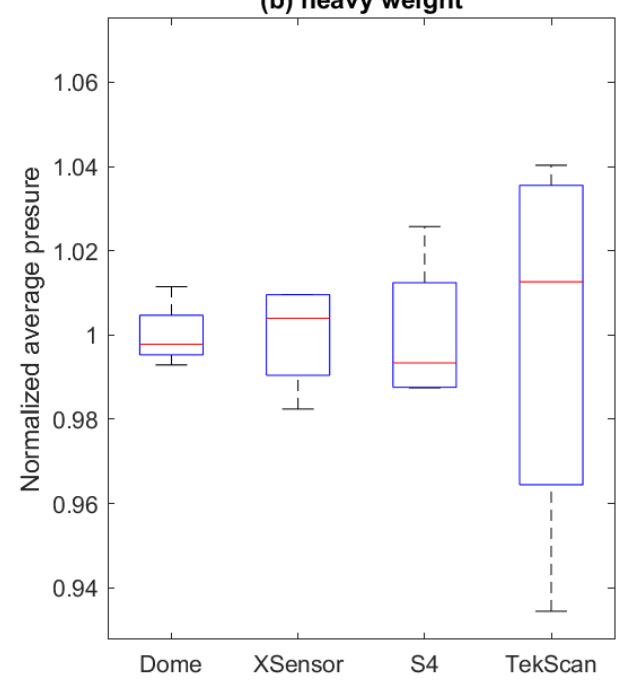

Figure 25 - Normalized average pressure for light and heavy weight in short-term experiments. 


\subsubsection{Long term experiments}

For the long term experiments, three metrological properties were explored: rise time, total creep and average drift. Table 1 shows the results of these metrics for four different technologies using the light weight (0.28 psi), similar to the neonate model explored in [43], and Table 2 shows the results considering the heavy weight $(0.37 \mathrm{psi})$, similar to the adult model, also explored in [43].

The rise time of the developed "Dome" PSM technology was far faster than all other technologies. For the light weight, the developed technology presented a rise time of 1.40 seconds. The second best technology was the S4, with a rise time of 17.53 seconds, then Tekscan with 24.47 seconds, and XSensor, with 143.35 seconds. For the heavy weight, the developed technology has a rise time of 1.43 seconds, followed by Tekscan, with 44.32 seconds, then S4 sensor, with 71.50 seconds, and then Xsensor, with 143.38 seconds. These results are elaborated upon in Section 4.4.

The total creep is computed using the signals measured from thirty minutes from the time in which the weight was applied, to eleven hours. A window size of sixty seconds is used to compute the mean pressure at thirty minutes and eleven hours. Following Equation 2, the mean at thirty minutes is subtracted from the final mean (at eleven hours), and divided by the mean pressure between thirty minutes and eleven hours. This provides the total creep shown in Table 1 and in Table 2. The total creep for the developed sensor is the smallest across all technologies for both light and heavy weights. The total creep for the developed technology is $1.63 \%$ for the light weight and $1.47 \%$ for the heavy weight. Tekscan was the second best technology, with a total creep of $4.33 \%$ for the light weight and $8.24 \%$ for the heavy weight. S4 was the third best technology, with $8.95 \%$ for the light weight and $15.92 \%$ for the heavy weight. The XSensor was the technology with the highest total creep, with $16.10 \%$ for the light weight and $16.11 \%$ for the heavy weight. 
The change in creep was also computed using a window size of one hour, where the last and first sixty seconds of pressure within each hour of measurement are used to compute the change in creep. Figure 26 shows the results for the developed Dome technology, and for the Xsensor, S4, and Tekscan commercially available technologies. A common pattern found in this figure is that the largest change in creep occurs in the first hour and continually decreases over time. The only exception is for the $\mathrm{S} 4$ sensor when the light weight is used. This anomaly is discussed in Section 4.4. This figure also enforces the results in Table 1 and Table 2, where the computed change in creep is the smallest for the developed Dome system, followed by Tekscan, and then the S4 and XSensor PSM technologies.
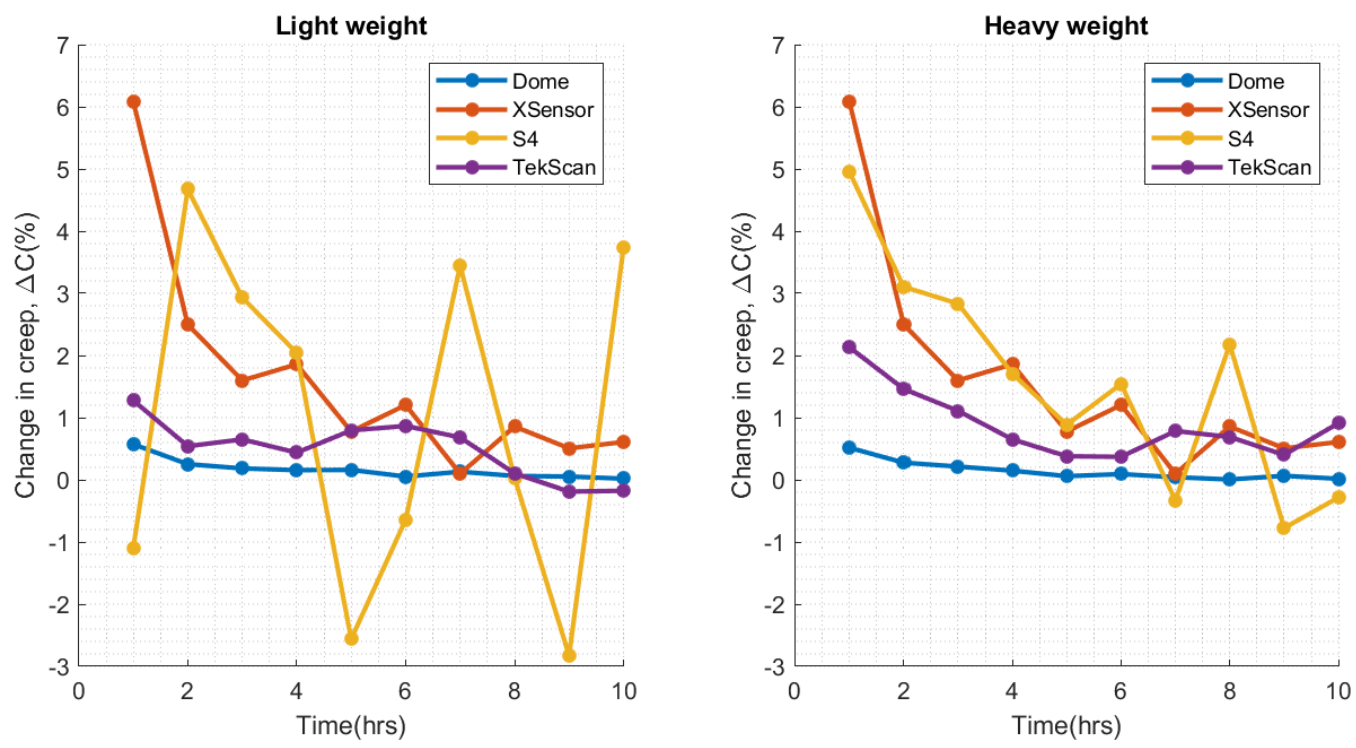

Figure 26 - Change in creep over time using window size of one hour.

The percentage drift computes the width of the distribution of the measured signal around a given mean. A window size of one minute is used here, where the standard deviation of the samples are divided by the mean across those samples and multiplied by hundred. Figure 27 shows the computed percentage drift over time using a one minute sliding window for the four PSM 
technologies. The average drift is computed using the mean across the computed windows, and a summary of these results can be seen in Table 1 and Table 2. The S4 presented the highest drift over all other technologies, with an average of $16.17 \%$ for the low weight, and $7.61 \%$ for the heavy weight. XSensor has an average drift of $4.33 \%$ for the light weight and $16.11 \%$ for the heavy weight. Tekscan and the developed technology have the smallest drift, requiring an inset figure with expanded y-axis in Figure 27 to effectively visualize the drift. Tekscan has an average drift of $0.31 \%$ for the light weight, and $0.30 \%$ for the heavy weight, whereas the developed technology has an average drift of $0.08 \%$ for the light weight, and $0.07 \%$ for the heavy weight.


Figure 27 - Percentage drift over 11 hours for the light weight (left) and heavy weight (right)

\subsection{Discussion}

The main goal of this chapter is the characterization of the metrological properties of the developed Dome PSM technology and a comparison with existing technologies. It was decided to repeat the 
same experiments on all devices here rather than using measured data from previous studies, such as [43]. One of the reasons for this is that the duration of the recorded signals do not match with other studies; additionally, possible differences in the applied weight and weight distribution on top of the sensors may have led to differences in measured metrological properties. While every attempt was made to replicate the experimental protocols used in [43], due to the limited size of the prototype sensor, a much smaller surface area for the applied weight was used. For example, in the case of the S4 PSM, only six out of 73 sensors were in contact with the applied weight. Therefore, it was decided to only use the sensors in contact with the applied weight for computing metrological properties whereas all sensels within the entire PSM were used in [43]. For this reason, some of the results differ from those previously found in other studies. For example, there is a discrepancy in the $\mathrm{CoV}$ found in this study and the values found in [43].

In terms of the short term experiments, it was found that the developed technology is better when considering the heavy weight, and close to the best technology when considering the light weight. The Dome achieved a CoV of $1.14 \%$ for the light weight and $0.8 \%$ for the heavy weight, which indicates that this technology could be used for repeated times with great agreement across different measurement intervals. To emphasize these results, the equipment with the closest result is the XSensor, which was acquired in the last year as a state of the art mattress available in the market (at a cost of $\sim \$ 30,000$ ). The $\mathrm{S} 4$ sensor presented the third best $\mathrm{CoV}$ for the short term experiment, even though is an older equipment. However the results related to the S4 sensor might not be reliable for the short term experiment because of the range of operation in which the sensor works. As it is an old technology, the resolution of the sensor is not even close to the current technologies, as well as the age of the material is much higher than all other devices, including the developed technology. For example, the resolution of each sensel appears to be $2^{8}$ bits. With the 
weights being applied to the mattress, there will not be a great variability on the sum of the recorded signals, which might limit the $\mathrm{CoV}$, not because of the quality of the sensor, but the lack of resolution. An empirical analysis on the signals easily leads the observer to conclude that the S4 Sensor is the worst against all other sensors. An example of this can be seen in Figure 23, where the variability of the signals from the S4 sensor is much greater than for the ones observed for the other sensors.

For the Tekscan PSM, which is a relatively new equipment, there is also a discrepancy with the results found in [43], where the computed $\mathrm{CoV}$ found was $2.93 \%$ for the neonatal model, and $3.67 \%$ for the adult model. In this study, the $\mathrm{CoV}$ of the Tekscan equipment was computed as $6.27 \%$ for the light weight and $4.79 \%$ for the heavy weight. These values are in accordance with the findings in [54], but are higher then those in [43], which represents a more recent study. For this reason, the same experiment was repeated three times to verify if the computed values were due to the experiment itself, or due to the equipment. Figure 28, shows the signals acquired from the Tekscan equipment over three different sessions (a-c). Figure 29 shows the normalized distributions across the three experiments. For the first experiment, the computed $\mathrm{CoV}$ for light and heavy weights were $20.69 \%$ and $7.75 \%$ respectively, for the second run they were $6.27 \%$ and $4.79 \%$, and for the third run they were $2.37 \%$ and $0.98 \%$. For this study, the results from the second run are reported since it does not exhibit the artifact seen in run three, and since the results are more consistent across the four repetitions for the second run.

Special care has been taken when positioning the weights on top of the mattress, so that the measurements would not be affected by the weight distribution. However, it is possible that small differences in weight distribution over the sensors could affect the performance of the Tekscan sensor. The disposition of the sensors and the material used in this technology may also 
have helped with the differences between each repetition, since the weight distribution could be more centralized in a given sensor. As both experiments for the light and heavy weights have similar discrepancies, it is assumed that the problem is not with the experiment, but with the device itself which may not provide reliable information when flat surfaces are used on top of the mattress.

(a)

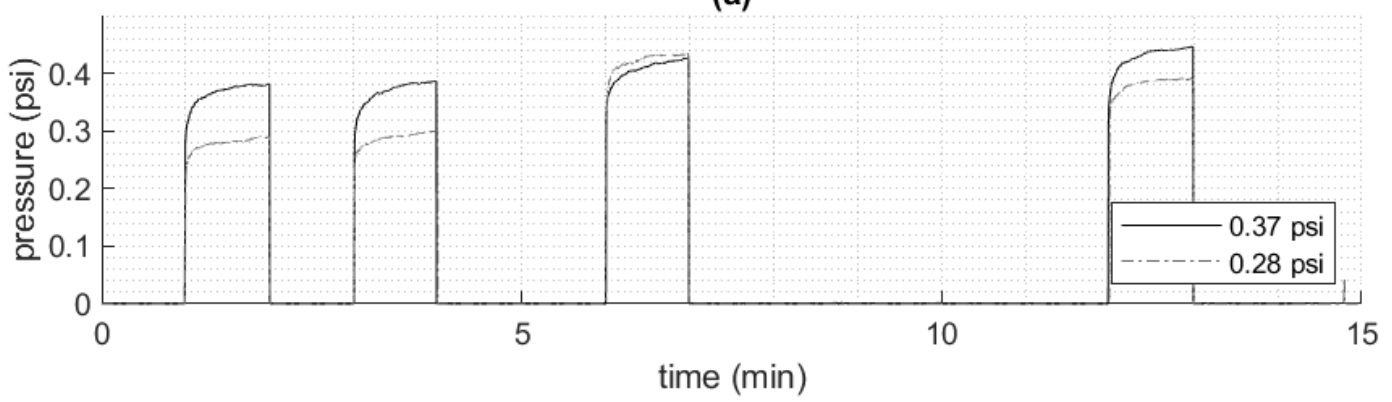

(b)



(c)

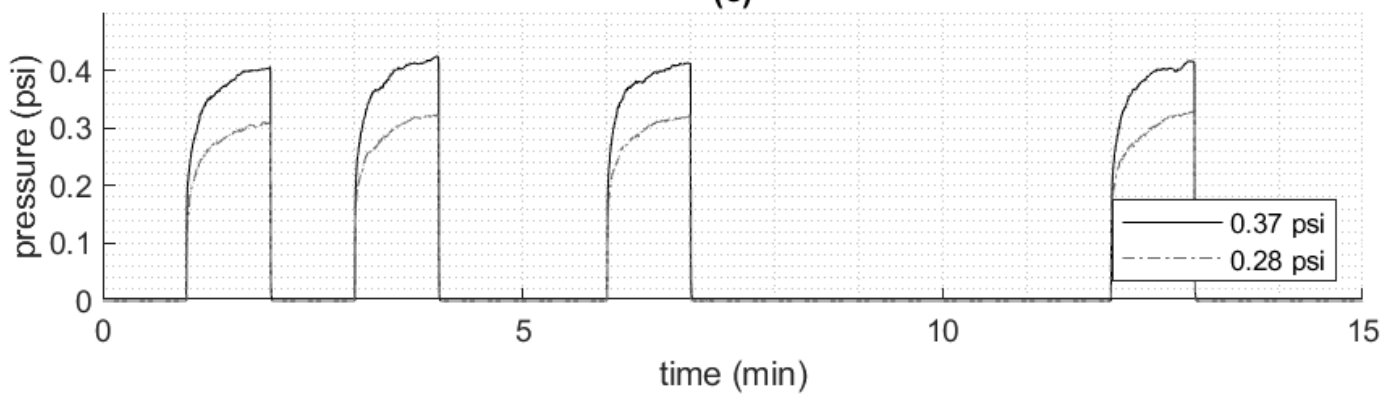

Figure 28 - Short term experiment repeated three times for the Tekscan equipment 

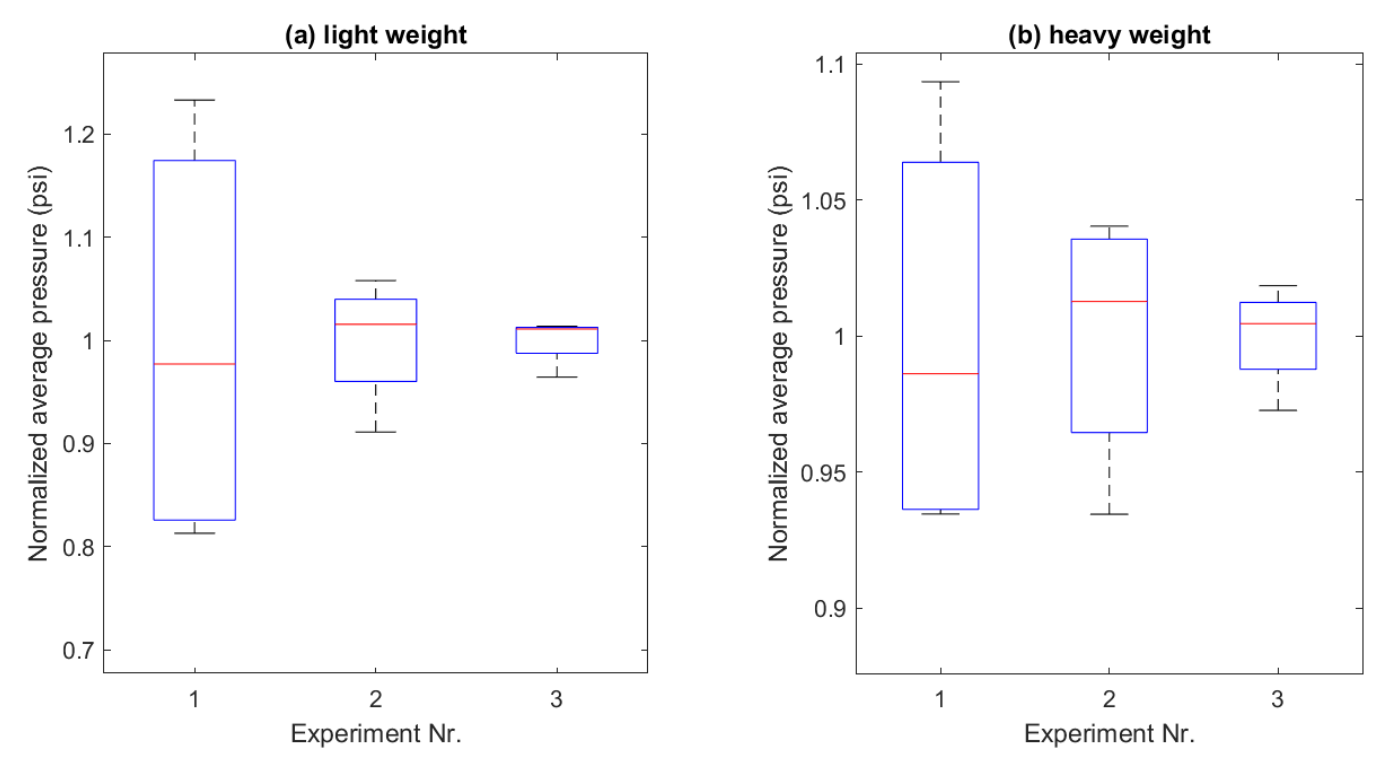

Figure 29 - Normalized average pressure for TekScan equipment over three trials.

As presented in Section 4.3, the developed Dome technology has the fastest rise time response. Figure 30 shows the first thirty minutes of the measured signals using the light weight (top), and heavy weight (bottom), with a line marking the ninety percent considered for the computation of the rise time. In both plots, a zoomed inset figure is provided for better visualization of the first $30 \mathrm{~s}$ of data. As can be seen, the Dome technology reaches the $90 \%$ of the steady state value (estimated at thirty minutes) after less than a second. However, the results presented in Table 1 and Table 2 show greater values than the ones seen in Figure 30. Due to the higher drift of the sampled signal, the $90 \%$ value could be achieved prematurely due only to erratic noise in the signal. For example, for the $\mathrm{S} 4$ sensor with the light weight applied, the signal varies from 0.16 psi to 0.28 psi in the first second. The ninety percent mark would be $0.252 \mathrm{psi}$, which means that for this very noisy signal, the rise time of less than one second is observed. To compute the rise time for all technologies, the signals were filtered with a moving average window of one second and upsampled to $600 \mathrm{~Hz}$, giving a better estimate of the rise-time due to the higher sampling rate. 
Due to the moving average low pass filtering, the transient time to go from baseline to steady-state increases, causing the rise time to be larger. The advantage of this procedure is that signals with higher variability will have a more robust estimate of the rise-time because it would be computed with signals with attenuated noise. For signals with higher variability, even the moving average window of one second is insufficient to eliminate all drift-related interference in the rise time analysis. On the other hand, the resulting signal will not interfere for most of the sensors and will better represent the actual rise time. The signals shown in Figure 30 are the original signals normalized by the theoretical pressure applied $(0.28 \mathrm{psi}$ and $0.37 \mathrm{psi}$ for light and heavy weights respectively). Figure 31 shows the signal smoothed using a moving average using rectangular window of one second. Note that the variability of the signals decreases. It is also possible to see that the signals are stretched in time, which is clearly seen at first two seconds of the Dome line. 

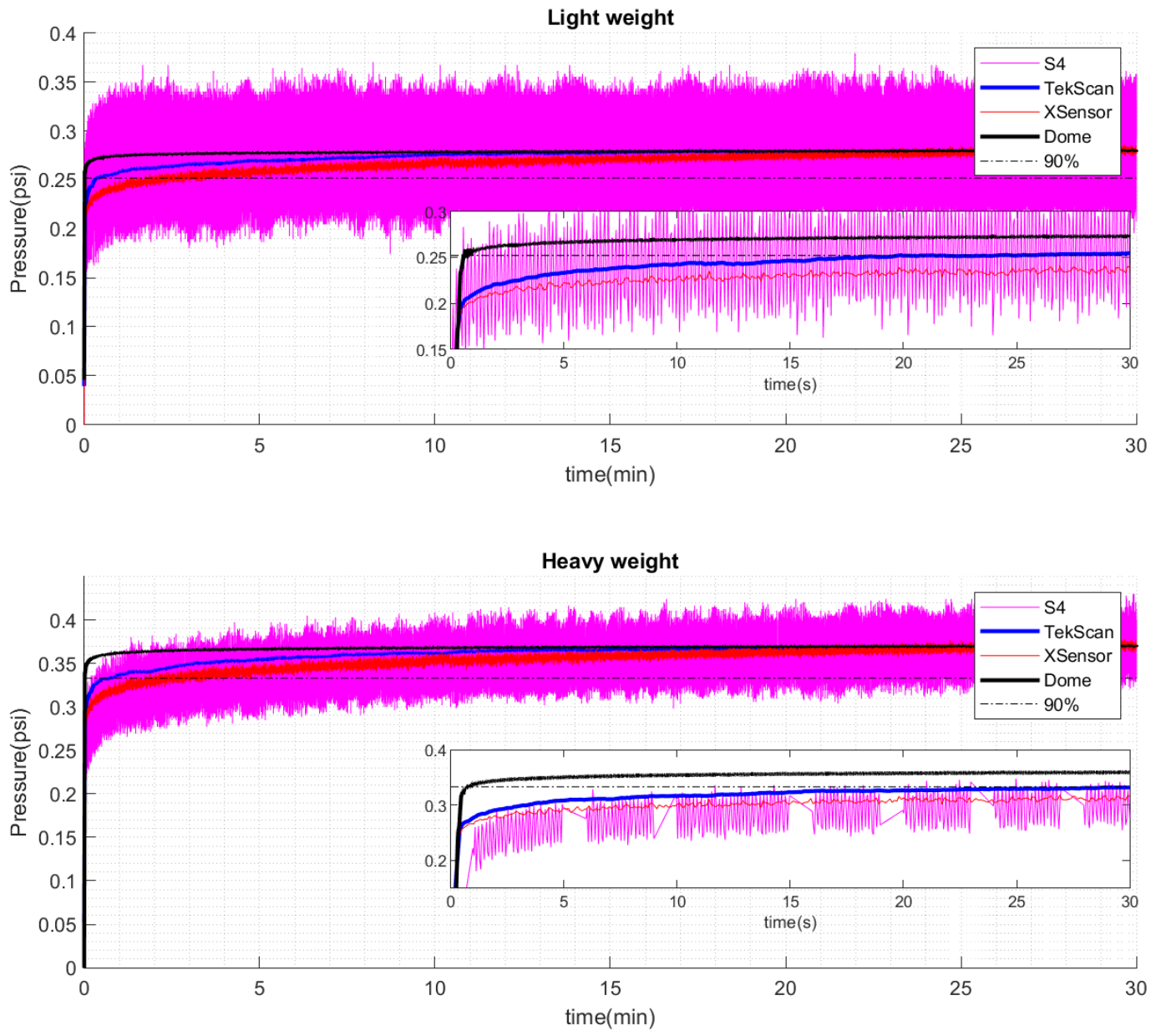

Figure 30 - Rise time analysis of the four technologies for light weight (top) and heavy weight (bottom) 

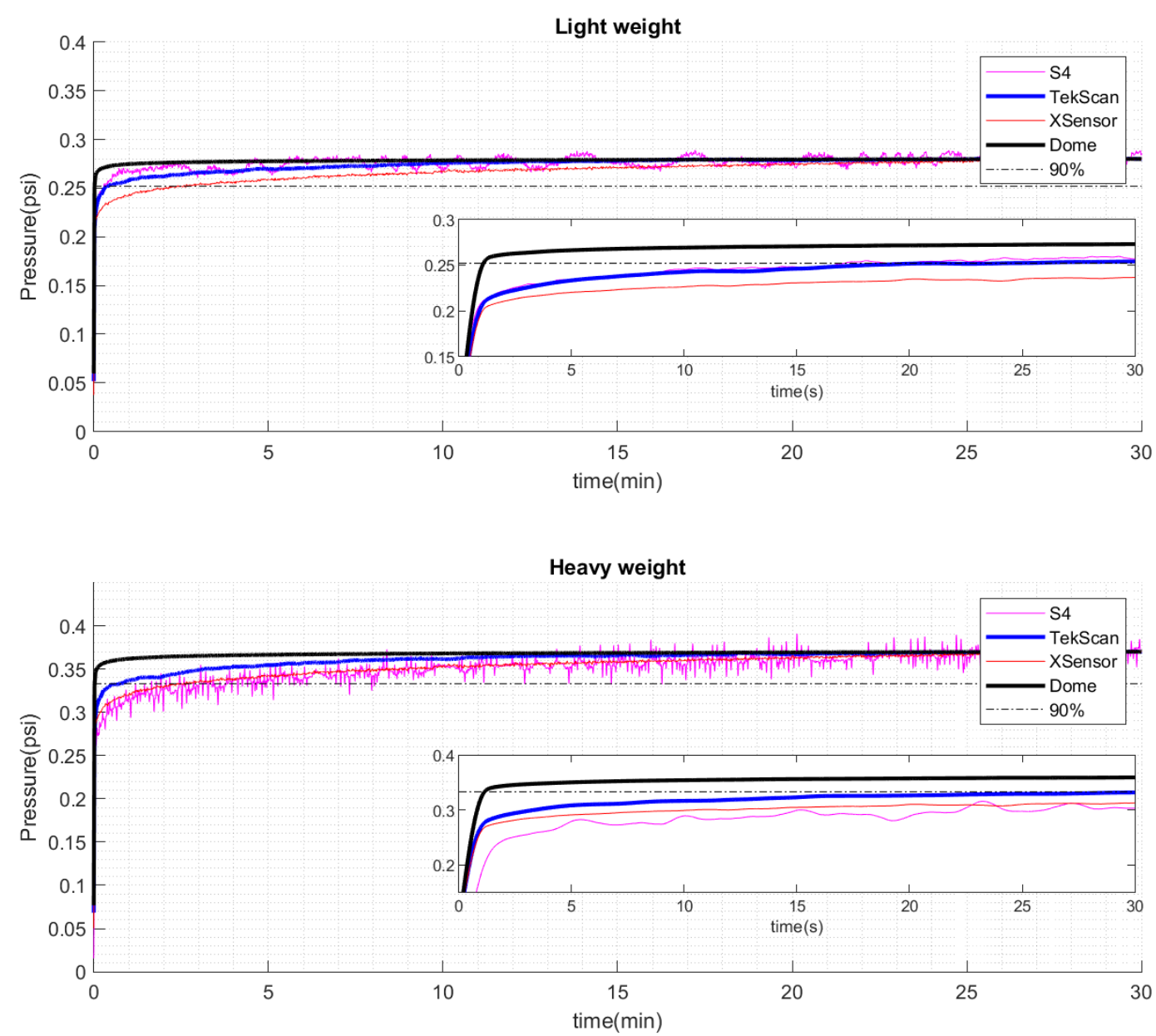

Figure 31 - Rise time analysis using the filtered signals for the four technologies for light weight (top) and heavy weight (bottom)

By looking at the signals measured in the experiments performed in this chapter, it is empirically possible to draw conclusions about the quality of the different technologies. For example, in Figure 23 (short term experiments) and in Figure 30 (long term experiments), it is possible to see the higher variations of the S4 technology, followed by the XSensor, and then Tekscan, and the developed Dome technology. This is confirmed by the computed Average Drift values. The average drift of the Tekscan, which presented the second best results, is 3.63 and 4.26 times higher than for the developed technology for the light and heavy weights, respectively. 
The change in creep over time shown in Figure 26 reinforces the results from Table 1 and Table 2, where the smallest changes are observed for the developed system when compared to the commercially available technologies. Because of this, there are indications that this technology is suitable for long term monitoring, where the smaller changes in signals over time would produce a more reliable signal.

As mentioned at the opening of this Section, there are some differences between the results found in this study and the results found in [43]. These may be due to differences in the experimental setup. Here, a metal plate was used beneath the weights so as to evenly distribute them over a fixed area. Also, only the sensors around the plate were considered, whereas in their study a neonate and adult model were used and all sensors were considered in the computations of the metrological properties. Furthermore, differences in signal preprocessing may have impacted the measured metrological properties. For instance, the average drift of S4 sensor in this study was around $7.61 \%$ for the heavy weight and around $16.17 \%$ for the light weight, whereas the results from [43] indicated an average drift of $0.03 \%$ for the adult model and $0.61 \%$ for the neonate. However, the authors in [43] pre-processed the signals from S4 using the average across twenty samples, which resulted in a new signal resampled at one hertz. This caused the variability of the signal to drastically decrease since averaging of these samples is equivalent to low pass filtering.

\subsection{Conclusion}

This chapter compared the metrological properties of the developed system with three commercially available products - S4, Tekscan and XSensor. The metrological properties were computed using both short and long term experiments. For short term experiments, light and heavy weights were applied to the top of the pressure mats for four repetitions. The mean over fifty seconds was used to compute the CoV. For long term experiments, light and heavy weight were 
applied to the top of the PSM for a minimum period of eleven hours. Rise time, average drift and creep were computed using these signals. It was shown that the developed "Dome" sensor has better metrological properties when considering the long term experiments, and performed better than all other technologies for the short term experiment using the heavy weight. On the whole, the new technology appears to have better metrological properties than all three commercially available PSM technologies evaluated here.

In the next chapter, the developed Dome PSM technology is demonstrated to be suitable for the estimation of respiration rate, a form of patient monitoring. 


\section{Evaluation of Breathing Rate estimation using the prototyped}

\section{sensor}

\subsection{Introduction}

In this chapter, an experiment with a subject laying atop of the prototyped device and breathing at a controlled rate is explored. The objective of this experiment is to evaluate the applicability of the implemented device to monitor a patient in an unobtrusive way.

\subsection{Methods}

The prototyped sensor is positioned on the ground and connected to a computer for data logging. The silicone layer is positioned on top of the flexible PCB, and the cavities are aligned with the 16 individual sensors. Next, a metronome implemented on MATLAB is configured to beep every 5 seconds, corresponding to a breathing rate of twelve breaths per minute, and to beep every 3 seconds for the breathing rate of twenty breaths per minute. The subject then lays in a supine position, with the upper back on top of the prototype PSM and the arms placed alongside of the torso. The subject is then instructed to regulate their breathing rate according to the metronome. When the subject hears a beep, he starts an inhale, followed by an exhale until the next beep is heard, repeating the breathing cycle every time the beep is heard. To avoid motion artifacts, the first thirty seconds of the recording are excluded from the analysis. The subsequent sixty seconds of the recording data were used to compute the time between breathing cycles.

\subsection{Results}

Using the recorded data and the methodology described in section 5.2, it was possible to compute the sum of all sensor recordings. Figure 32 shows the computed total pressure of a subject laying 
on top of the prototype and breathing using an audible metronome, in which the minimum and maximum common ranges of human breathing rates were simulated. The solid line represents the sum of all raw sensor recordings in counts, without signal conditioning. The triangles show the peaks found using the Matlab function findpeaks(). The times between peaks were used to compute the breathing period and breathing rate. As can be seen, there are some artifacts in the original signal for both slow and fast breathing rates, which may be due to noise or even related to how the subject is moving on top of the prototype. This artifact might lead to larger fluctuations of the time intervals found by the peak function. Figure 33 shows the original signals filtered by a moving average with a rectangular window of one second, which eliminates all the artifacts from the original signal, yet retaining the information pertinent to the breathing rate. The advantage of filtering the signals is that the fluctuations around the peaks decrease creating a more precise estimation of the breathing rate.

Figure 34 shows the estimated breathing intervals from the time series data. The blue line represents the estimation using the original signal, which has artifacts, and the red line shows the breathing intervals using the filtered signals. As can be seen, the deviations around the mean of the breathing rate are larger when using the original signal. The filtered version produces a more stable response with smaller deviations around the true breathing rate. 

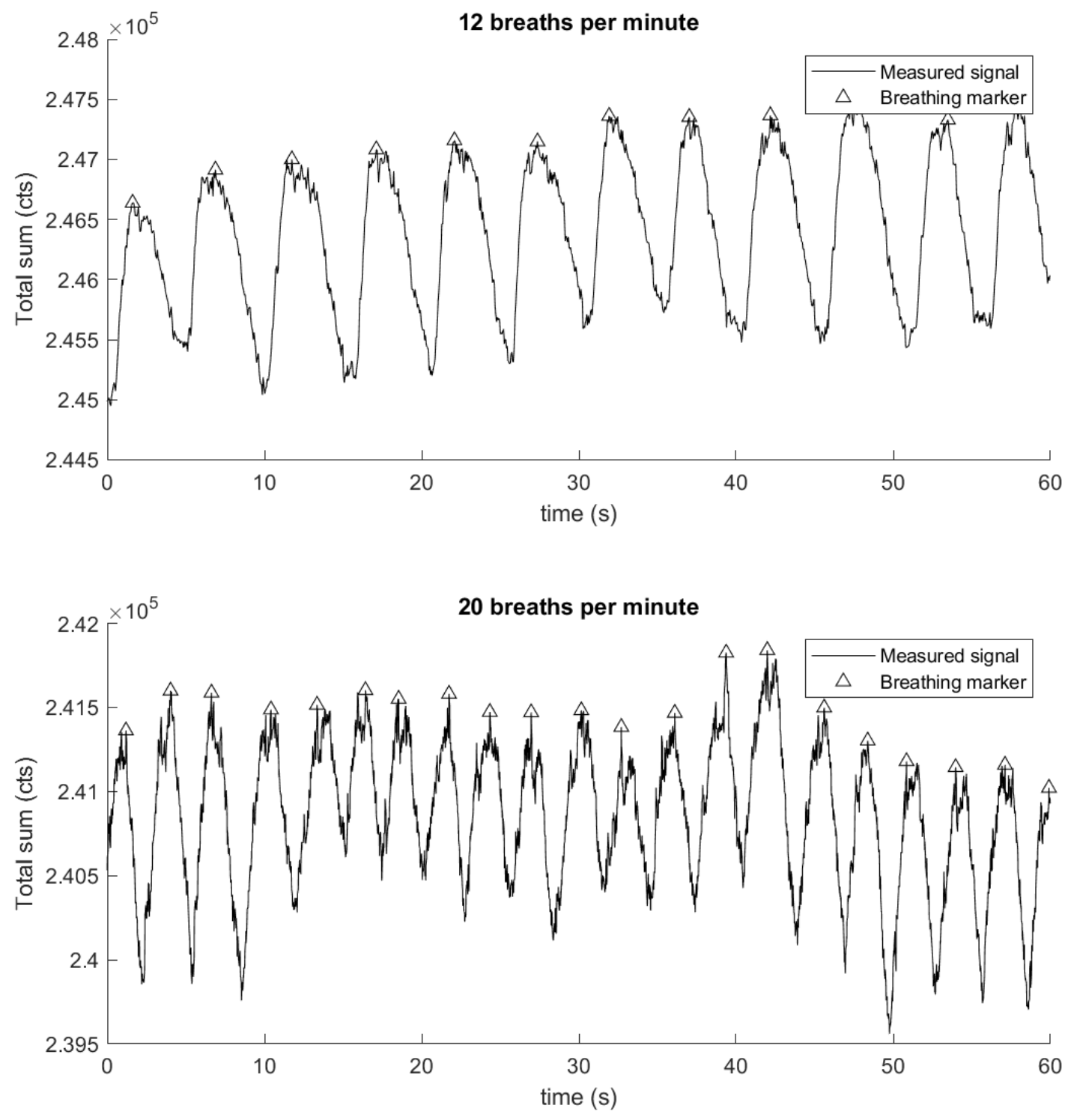

Figure 32 - Total pressure over time of a subject laying on the PSM prototype in supine position. Respiration rate was controlled with a metronome. The breathing rate of the top figure was set to 12 breaths per minute and the bottom figure was set to be 20 breaths per minute. 

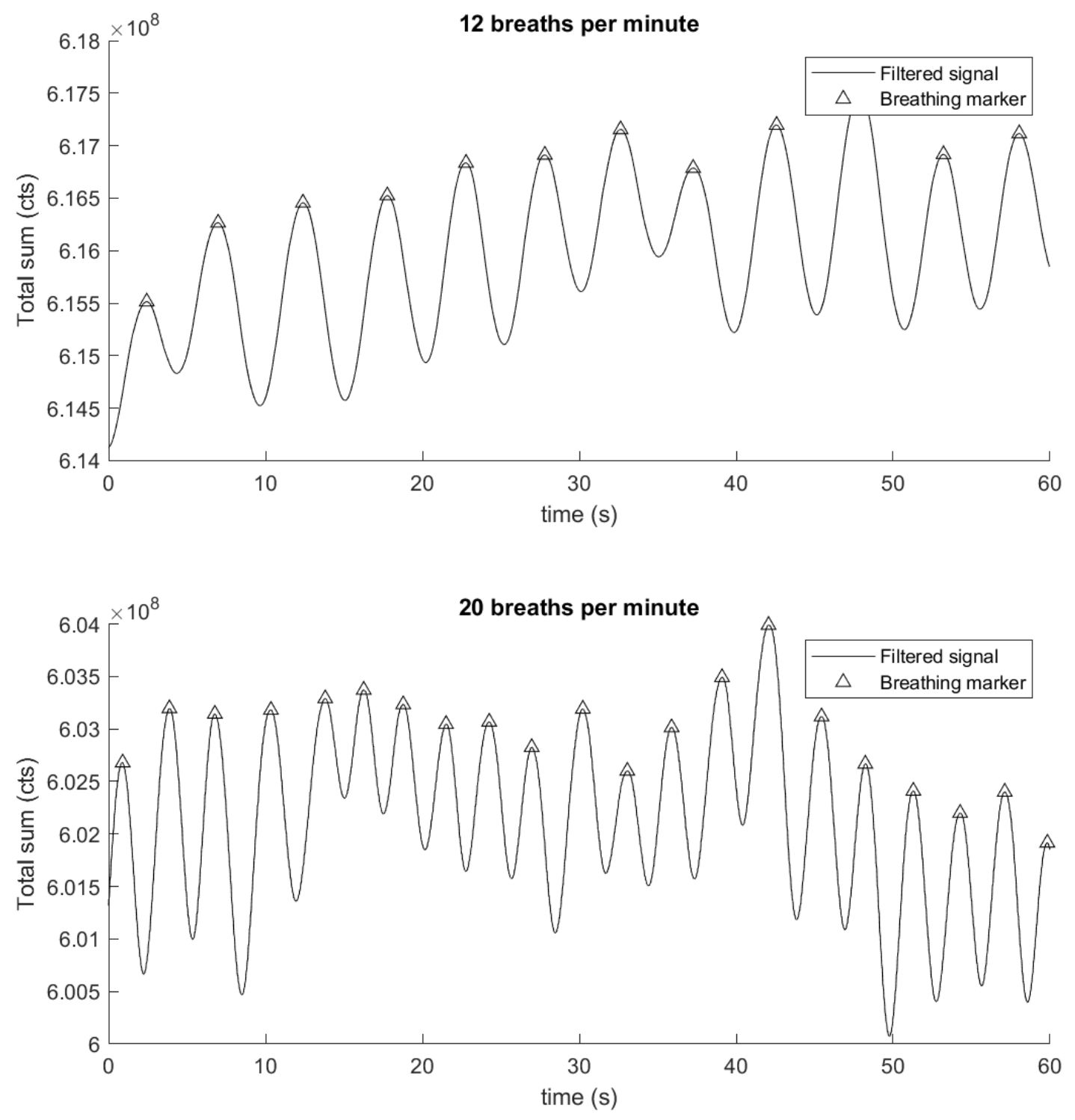

Figure 33 - Filtered total pressure over time of a subject laying on the PSM prototype in supine position. Respiration rate was controlled with a metronome. The breathing rate of the top figure was set to 12 breaths per minute and the bottom

figure was set to be 20 breath 

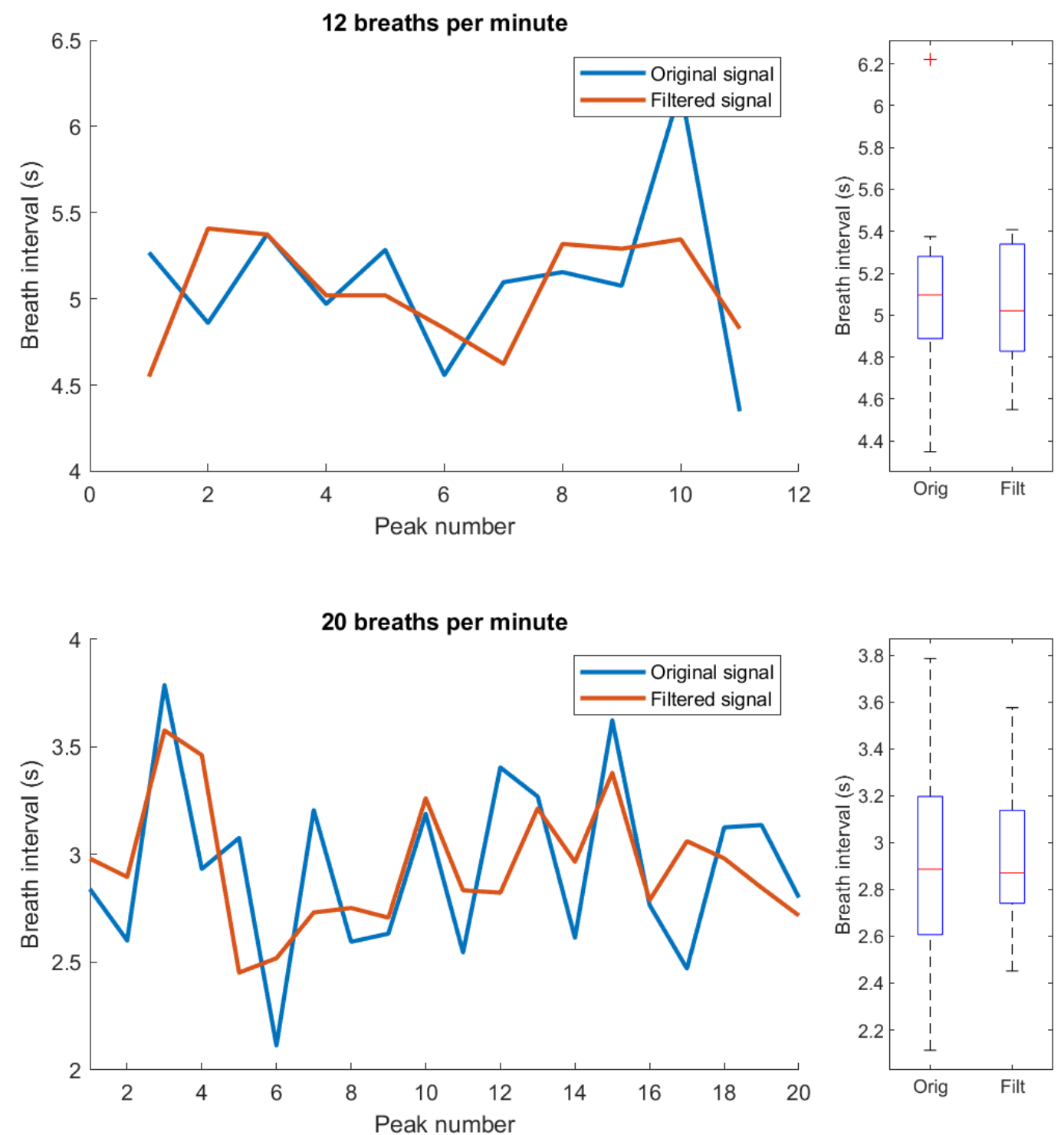

Figure 34 - Breath intervals of a subject laying on the developed prototype in supine position. Signals extracted from the original signals and from the filtered signals using a $1 \mathrm{~s}$ rectangular window moving average.

\subsection{Discussion}

As can be seen in Figure 32, the breathing rate is clearly recognizable in the measured contact pressure signal. In this case, the periodic waveform is caused by the displacement of the thorax 
while the subject is breathing. Because of this movement, there is a weight redistribution, which can be measured by the pressure sensels. Since the area of the prototype is small when compared with the thorax area, it is possible to use all sensors of the device to estimate the breathing rate based on centralized weight distribution. In cases where the pressure sensitive mat covers all parts of the body, a subset of sensors must be selected. A number of algorithms have been proposed to estimate the breathing rate using only a selected range of sensors [36], [55], [56]. These sensel selection strategies include the assessment of sensor signal variability using a sliding window and computing the reliability metric of a given sensor for breathing rate estimation. One of the limitations of this study is that only one subject was used to validate the ability to estimate breathing rate. Variations between subjects, such as the effecti of different body mass indexes, will require validation on a larger and more diverse population of subjects. Another limitation on the breathing estimation is that the gold standard considered in this study is the metronome, which does not necessarily represent the real breathing intervals (i.e. the subject's actual inter-breath times are expected to vary somewhat). This problem is minimized because the mean of the intervals are computed using a one minute window. Even with small variations on the breathing intervals, an accurate breathing rate estimation was reached when considering the metronome intervals.

\subsection{Conclusion}

The prototyped design provided quantitative information that allows the extraction of breathing rate through changes in pressure of a person laying on top of the device. The raw measurements from all sensors were computed as a total pressure without linearization nor selection of the best individual sensors for breathing rate extraction. Even so, a standard algorithm for peak detection using Matlab successfully extracted the time intervals of the breathing pattern, which accurately 
provided the breathing rate controlled by a metronome. A simple moving average filter was used to mitigate artifacts from the total pressure, producing a more precise estimate of the breathing intervals of a subject laying atop the prototype. This result achieves the stated objective of this chapter, which was to demonstrate the applicability of the developed Dome PSM technology for unobtrusive patient monitoring.

The next chapter does not emphasize the characterization and possibilities of the implemented system, but rather suggest an improvement in the communication protocol that can significantly increase the sample rate using an adapted $\mathrm{I} 2 \mathrm{C}$ protocol, or even double the number of sensors keeping the same sample rate. 


\section{Analysis of an alternative communication protocol}

\subsection{Introduction}

The current off-the-shelf infrared sensor used in this project utilizes the $\mathrm{I} 2 \mathrm{C}$ protocol for communication with the microcontroller. $\mathrm{I} 2 \mathrm{C}$ is a standard communication protocol that is used by a broad range of devices, including sensors with embedded digital communication. Usually, one device works as a master, which controls the slave devices connected to the network. Ideally, each of the slave devices would have a unique address, so that the master can request information from a specific sensor. Since each sensor has an individual address, only one sensor replies to a request at a given moment, avoiding data collision on the $\mathrm{I} 2 \mathrm{C}$ bus. Some slave devices permit the configuration of the address, but this is not common in low cost sensors. Thus, extra circuitry must be added to allow switching of the SCL and consequently avoid data collision. To communicate with a slave, the master begins a request by sending the start bit followed by the address of the slave and a bit indicating whether this is a read or write bus cycle. The slave then acknowledges the master with a bit response. In the case of the VCNL4020, to read the sensor, the master starts the communication with the slave address, followed by a write bit, then the slave sensor acknowledges it is ready. The master then indicates the register it wishes to read from, then the sensor acknowledges. To read the values within the requested register, the master starts the communication, followed by the slave address and a read bit. The sensor then acknowledges and writes the contents of the requested register. Finally, the master acknowledges that it received the data and stops the transmission.

While this is a well-known communication protocol that is easy to use, it is inefficient when multiple sensors are used and only a few registers from each sensor are read. For example, each 
time a register read request is executed with the VCNL4020 proximity sensor, the first two bytes sent are the address of the sensor and the register to be read. Considering that only two bytes are read from the sensor, then the useful information transmitted on the bus represents only $50 \%$ of the total message. This procedure is replicated throughout all the sensors, exacerbating the problem.

The infrared sensors available in the market using the $\mathrm{I} 2 \mathrm{C}$ communication protocol often do not offer the possibility to configure its $\mathrm{I} 2 \mathrm{C}$ address. When this feature is offered, it is not completely possible to change the I2C address, but only to set one bit through a specific hardware pin, making it possible to have two different addresses. In other words, it would be possible to connect two sensors at the same $\mathrm{I} 2 \mathrm{C}$ bus, while achieving different addresses by wiring the address pin of the first sensor to ground, and to VCC for the second sensor. Again, when there is a need for more sensors in a single design, it is not possible to wire them without the use of additional hardware such as the multiplexers, as used on the prototype Dome PSM.

The standard maximum number of slaves allowed to be connected to the $\mathrm{I} 2 \mathrm{C}$ bus is 127 due to its 7-bit address. Even if the manufacturers permit the I2C sensor address to be configured, it would not be possible to connect more devices to the same bus without additional hardware. Also, each communication with a sensor would be needed to start a new connection with the slave, requiring the master to send the device address and the register to be read. Again, the efficiency of such a system would be limited to $50 \%$ when considering the reception of two bytes. As a result, and considering that the communication speed is finite, there is a limitation either in the number of sensors or the sample rate used in the system. So there is a need for more efficient protocols that could increase the sample rate or the number of sensors connected to the same communication bus. In this chapter, a new alternative protocol adapted from $\mathrm{I} 2 \mathrm{C}$ is proposed for multi-sensor designs, 
in which the efficiency is increased by almost one hundred percent when large amounts of sensors are used in the same system and using the same communication bus.

\subsection{Methods}

I2C is the protocol used in the prototyped PSM in this thesis since it is required by the hardware architecture of the VCNL4020 proximity sensor used. However, a new protocol could be used to achieve better efficiency. As an alternative protocol to allow the use of multiple sensors and ease of operation, it would be interesting to have a protocol where the clock and data bus are the same for all sensors, and two additional pins are used in a shift register fashion. A single read cycle initiated by the microcontroller would result in a burst read, whereby each sensor provides data in sequence. The first pin, slave enable (SE), would receive a pulse signal from the microcontroller (in the case of the first sensor) at the same time that a register reading request is executed through I2C. All sensors would receive the command with the address and the register to be read, but only the sensor which receives the pulse at the SE pin would return its register value to the $\mathrm{I} 2 \mathrm{C}$ bus. When the sensor finishes writing the register values, it will send a pulse to the second pin, master enable (ME), used in this alternate protocol. The ME pin of the first sensor is then connected to the SE pin of the subsequent sensor in a daisy-chain configuration. As soon as the second sensor receives this pulse, it starts to write the register values to the $\mathrm{I} 2 \mathrm{C}$ bus. This procedure is then repeated for all sensors until the last sensor is reached. The ME pin of the final sensor would then be connected to an input pin on the microcontroller, which recognizes the signal pulse and stops the (modified) I2C clock bus and consequently stops reading from the sensors. Figure 35 illustrates the connections needed for the proposed communication protocol. Note that no multiplexers or switches would be required to connect all sensors to a single (modified) I2C bus. While the cost of the sensor would increase because of the additional pins and complexity, we avoid the need for 
extra circuitry and the communication speed would be increased. This alternate protocol would have an increased efficiency, where either the sample rate or the number of sensors could be increased. The efficiency of this protocol could be represented by the following formula:

$$
\text { Efficiency }=\frac{\text { Bytes read } * \text { Number of sensors }}{\text { Bytes read } * \text { Number of sensors }+ \text { Bytes written }} * 100
$$

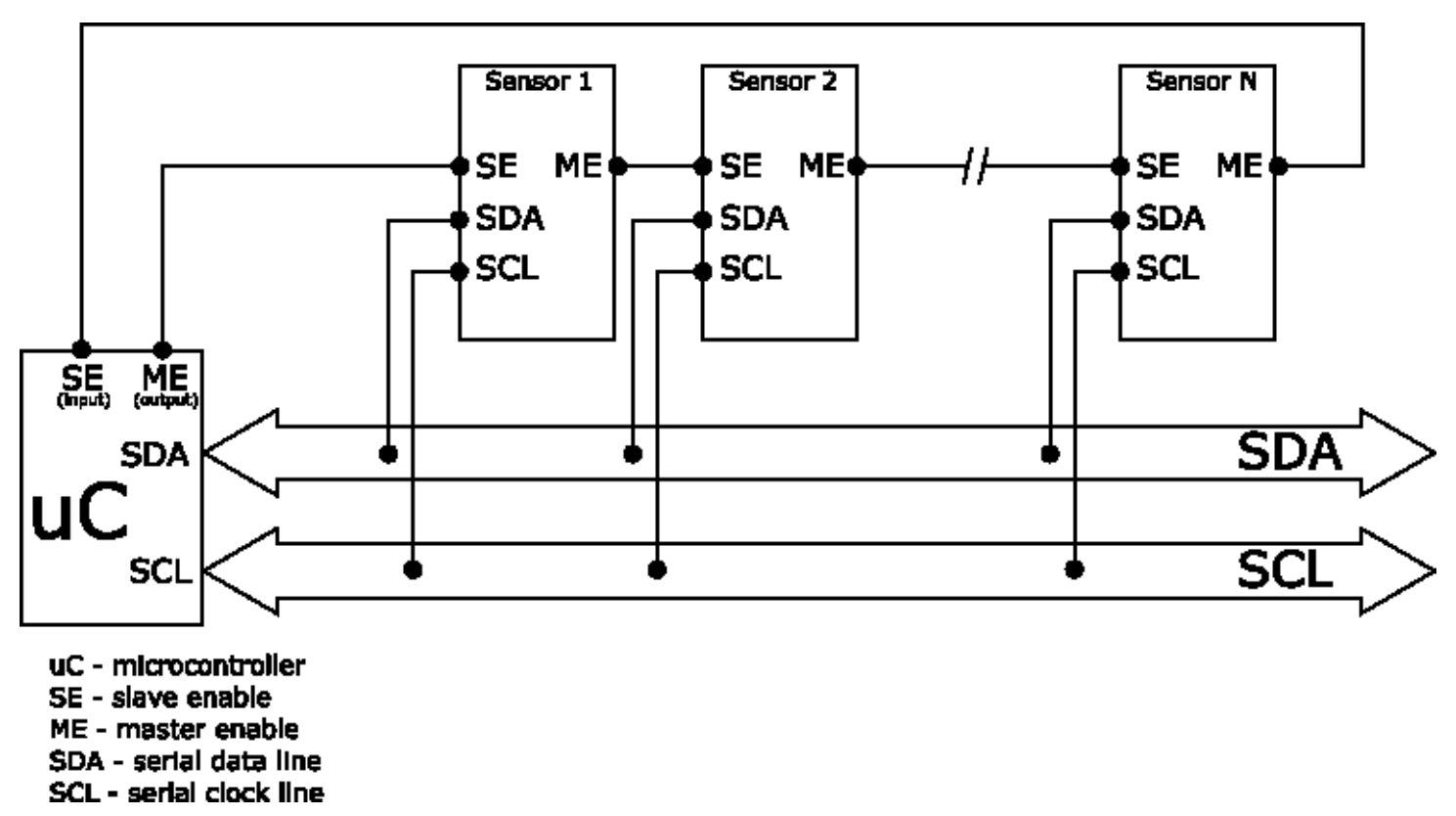

Figure 35 - Circuit representation of the proposed communication protocol.

\subsection{Results}

If only a single sensor is used, then the efficiency of the alternate protocol would be the same as the I2C. As the number of sensors increases, the efficiency of the alternate protocol will tend to one hundred percent. Figure 36 shows the efficiency considering two bytes written by the microcontroller (address and register to be read), and one, two, and 10 bytes returned per sensor. As can be seen, the alternate protocol would have significant improvements over the standard I2C protocol. For the VCNL4020 sensor, only two bytes related to the measured proximity are read 
per sensor. Figure 37 shows the fold increase of the alternate protocol in relation to the $\mathrm{I} 2 \mathrm{C}$ protocol. It can be seen that as the number of sensors increase, there will be a fold increase of one. In other words, the alternate protocol is able to transmit twice the amount of information at the same amount of time.

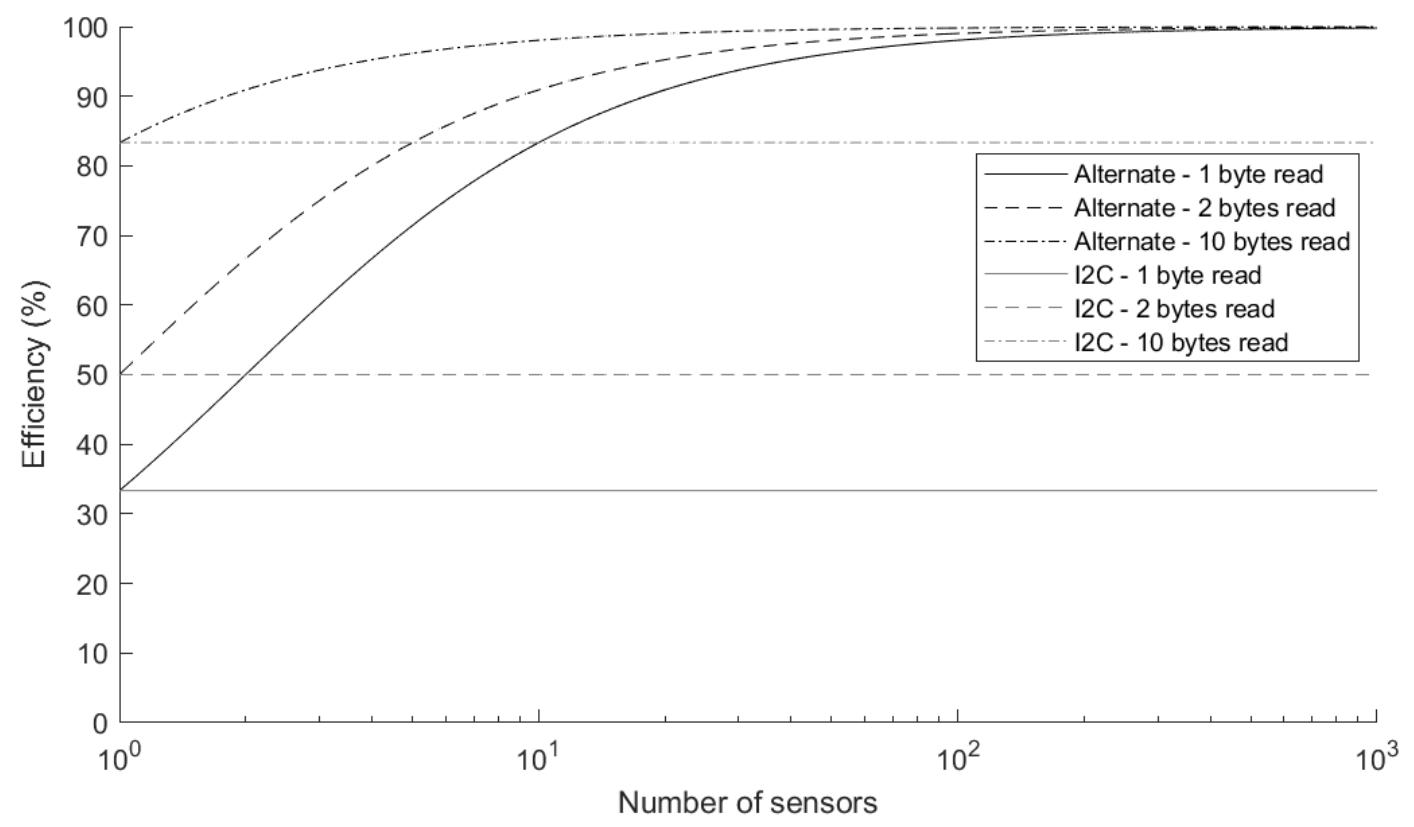

Figure 36 - Efficiency comparison between the I2C and Alternate protocol considering one, two, and ten bytes read. 




Figure 37 - Fold increase between the Alternate and I2C protocol considering that only two bytes are being read per sensor.

\subsection{Discussion}

As shown in section 6.4 , the alternate protocol provides advantages when compared with the available protocols used in digital infrared sensors equipped with I2C. The state-of-the-art VCNL4020 digital sensors already have benefits such as integrated voltage regulator, built-in emitter and infrared receiver, programmable current, and ambient light suppression, but they are made to be used individually, or in small quantities. As some applications might require a large number of sensors within a single design, such as for pressure sensitive mats, it is expected that the industry may wish to adapt the current technology or creates new ones, allowing the efficient simultaneous use of larger number of sensors in the same design. With the adaptation of current sensors with the proposed communication protocol, the overall cost of the final product can be 
reduced, and the industry can help with the deployment of pressure systems in clinical settings where money is a concern.

\subsection{Conclusion}

An adaptation of the $\mathrm{I} 2 \mathrm{C}$ communication protocol was presented in this chapter where the efficiency is improved to almost one hundred percent when more than one thousand sensors are used, and to eighty percent when ten sensors are used. For comparison, the XSensor LX100:36.36.02 PSM has 10,000 sensels in a single device, therefore it is not unreasonable to expect at least one thousand sensors to be included in a single design. Special emphasis was given to the situation where the address and the register to be read by the master is sent to the slave, and each slave replies with two bytes of information. This is the typical situation for reading from a low cost sensor, thus being the best situation for analysis. By implementing this protocol, it is possible to increase the sample rate or the number of sensors while keeping the advantages of the integrated circuit, which reduces the noise effect and allows the configuration of the sensor through software. 


\section{Thesis Summary and Future Recommendations}

\subsection{Conclusions}

The main goal of this thesis was the development and evaluation of a new pressure sensitive mattress technology using proximity sensors. The design of this technology employed off-theshelf integrated circuits, making it possible to utilize the technology in existing products. The main concept of this design is to send infrared light into a cavity and to measure the light reflected by the ceiling of the cavity. When force is applied to the cavity, a deformation of the material decreases the distance between the sensor and the cavity ceiling, causing the measured signals to increase. Thus, it is possible to convert the intensity of light measured by the sensor to applied pressure.

The VCNL4020 proximity sensor was selected as the emitter and receiver of infrared light as a means of measuring the distance from the sensor to the surrounding cavity. The advantages of this component is that all configurations related to the sensor are done through $\mathrm{I} 2 \mathrm{C}$ and it can thus be adapted to different products without any hardware changes. Also, the sensor has an embedded emitter and receiver, decreasing the size and allowing better replication across different sensors. In addition, the light emitted to the cavity is modulated, which avoid threshold biases from ambient light emissions, allowing a much cleaner signal with less interference from the environment. A series of silicone rubber products were used in the development stage, culminating in the final design consisting of a silicone layer manufactured with silicone rubber Ecoflex ${ }^{\circledR} 00-50$ from Smooth-on, with individual domes for each sensor. The walls of the domes are manufactured in black color, whereas the ceiling of the dome is made with white color, which reflects most of the infrared light emitted by the sensor. 
By adding weights to the top of the silicone layer and converting the values to a first order equation, an $R^{2}$ of 0.995 was reached. This indicates that the developed sensor has a linear relation to the pressure applied. Additionally, the metrological properties of the implemented system were compared to the metrological properties of existing commercially available technologies such as the S4, XSensor, and Tekscan PSM. It was shown that the CoV of the developed system is better than all compared technologies for the heavy weight, and only $1.14 \%$ for the low weight $(0.15 \%$ more than the best technology for this metrological property - Xsensor). In addition, it was shown that the developed system has better metrological properties than the other measured technologies in terms of rise time, total creep, and average drift, which could significantly improve the patient monitoring with existing algorithms, due to the reduced noise and faster response to stimuli.

In a proof of concept, the novel Dome PSM technology was shown capable of estimating the breathing rate of a person laying atop of the device. Lastly, the limitations of using the $\mathrm{I} 2 \mathrm{C}$ protocol for communication with the proximity sensors were discussed. An alternate communication protocol suitable for systems that utilize a large number of proximity sensors was proposed and was shown to double the efficiency of the default I2C protocol for systems requiring large numbers of sensors, such as a PSM.

\subsection{Summary of contributions}

This thesis has provided three main contributions. The first contribution being the development of a new pressure sensitive technology using proximity sensors and a compressible dome made of silicone rubber, which could be used in real-time applications. The second contribution being the evaluation of the implemented system in terms of its linearity, and the comparison of the metrological properties with existing technologies, showing improved performance in long term experiments when compared with existing technologies, such as rise time, total creep and average 
drift. The third contribution was the demonstration of the suitability for patient monitoring, in which the breathing rate was estimated while a subject laid on top of the prototype. This work also demonstrated that an improvement in the communication protocol could decrease the costs with sensor connectivity, and increase the sample rate avoiding the shaking between the controller and the sensor for each reading.

\subsection{Limitations}

The prototype version of the implemented system is composed of two main parts, which are the PCB and the silicone layer. Currently, the two parts are not permanently attached to each other. This was initially intended for testing distinct silicone layers, with different materials, forms, and colors. However, the baseline signals observed within the measurements change as the position of the silicone layer moves in relation to the sensor. This is not a problem for the preliminary evaluation of the sensor as the position does not change within a session. However, for final product and repeatability in clinical setups, the silicon layer would have to be attached to the PCB, avoiding the problems related to sensor-cavity alignment.

The VCNL4020 has a resolution of 16 bits, which can be dynamically maximized for a given design by changing the current applied to the emitter. This resolution is much better than the resolution of other existing technologies, such as the S4 sensor. However, the 16 bits of resolution are not likely to be used when measuring contact pressure in a PSM because either the sensor saturates (when too much current is applied), or the baseline signal (when there is no applied pressure) is too high. For example, when applying $110 \mathrm{~mA}$ of current to the sensor, the baseline measure can vary from around 10k to $20 \mathrm{k}$ counts, which makes only about 15 bits of resolution available for the difference in pressure. For the experiments performed in this thesis and considering the final silicone layer employed, a current of $110 \mathrm{~mA}$ was applied to the sensor, but 
there is an opportunity to explore the impact of smaller currents being applied for different weights using the same silicone layer. Also, it opens the opportunity to develop a new sensor to work in a smaller distance range, and that could be used in a closer distance than the current sensor, allowing the cavity to be smaller, and consequently cheaper and lighter.

In terms of spatial resolution, the current prototype may not reach the sensel density of other technologies, such as resistive and capacitive PSM. The dimensions of the existing proximity sensors are larger than the spatial resolution of high-end commercially available PSM, such as the one from XSensor. In addition, considering the Dome technology, the wall domes impose steric constraints on adjacent sensels, further limiting the spatial resolution of the Dome technology. However, the larger spatial resolution may be sufficient for many applications.

The experiments described in sections 3.3.2, 4.2, and 5.2, were performed only one time, and the results were provided based on these measurements. Repeating these experiments multiple times could provide better estimates of the results. Thus, future work in this area should consider repeated experimentation and the results described with a standard deviation.

\subsection{Recommendations for future work}

The work done in this project refers to a prototype sized four by four inches, which serves as a proof of concept and as reference for the capabilities of the described technology. A better proof of concept would be the exploitation of the technology in a full-sized mattress suitable for measuring an entire patient. Assuming such a device were available, the signals from individuals would be collected and compared with existing technologies of similar scale, using algorithms presented by previous and current students of our lab. This may provide better indicators as to whether this technology may be used and commercialized in clinical setups. 
The use of this technology was initially thought to be used as a device to be placed above or below a mattress. However, the sensors used provides the possibility of arranging the sensors in a variety of configurations. We are therefore not restricted to traditional array configurations. This could enable the use of this technology in different products, such as wheelchair, shoes, sport devices, and others. Thus, future studies in different areas could use the current technology to measure contact pressure and provide feedback from time-series signals. Also, there is the possibility of designing individual sensels, where a proximity sensor would be inside an individual cavity and connected to a microcontroller, allowing the user to install the sensor in configurable positions as needed. For example, these individual sensels could be used in the arms of a chair, and monitor the pressure differences between both arms. We demonstrated the potential for breathing rate estimation, but an investigation about the capabilities related to other vital signs, such as heart rate, could also be explored.

The current prototype is mainly composed by two parts. The PCB, with all components and circuitry, and the silicone rubber layer, which comprises the cavities for the proximity sensors. Even though this is sufficient for the proof of concept, there is a need to design the connection interface between the PCB and the silicone. As some applications require the device to be waterproof, it would be interesting to consider this aspect in the design stage, considering the materials used around the circuitry. In this project, silicone rubber from Smooth-on was used, and the prototype silicone layer was constructed in a non-specialized laboratory. For example, the mixture between the compounds A and B were done inside a container using common wood sticks. Due to the mixture and the viscosity of the material, air bubbles are formed within the silicone. The bigger bubbles usually settle to the surface of the silicone, but many small bubbles remain inside the silicone. To decrease this effect, a process employing suctioning of the air can be used, 
creating a vacuum around the container, and sucking the bubbles from the silicone. This process was performed using a small vacuum cleaner, but it was not strong enough to suck all the bubbles from the silicone. A new design could include the use of industrial tools and a variety of different materials. For example, considering the use of polyurethane rubber and other materials, using appropriate techniques during the manufacturing process.

As discussed in section 7.3, the silicone layer is not permanently attached to the flexible PCB. Because of this, changes in the measurements may occur between experiment if the silicone layer position changes with respect to the underlying PCB and sensors. This may complicate calibration of the device for multiple experiments. Future work may contemplate a solution to permanently fixing the flexible silicone rubber layer to the $\mathrm{PCB}$, thereby avoiding the relative movement in different experiments.

The experiments performed in this thesis used a small sized prototype of $10 \mathrm{x} 10 \mathrm{~cm}$. Experiments with full-size mattress could be performed to validate the performance metrics here described. In addition, the experiments were performed directly on top of the silicone rubber and no actual bed mattress was used. In the case of PSM, a variation of the experiments could also be performed, where the PSM would be positioned between the subject and a mattress. Similarly, the same experiments could be done with the PSM under the mattress. 


\section{Appendix A: Sensor characteristics}

Appendix A includes the characteristics of the VCNL4020 sensor used in the design of the developed technology

\section{A.1 VCNL4020 sensor characteristics}

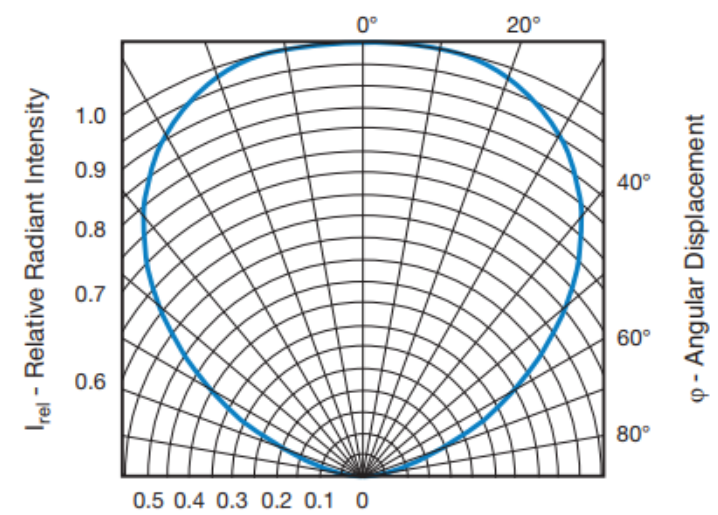

Figure 38 - Relative radiant intensity vs. angular displacement of the emitter (reproduced from [57])

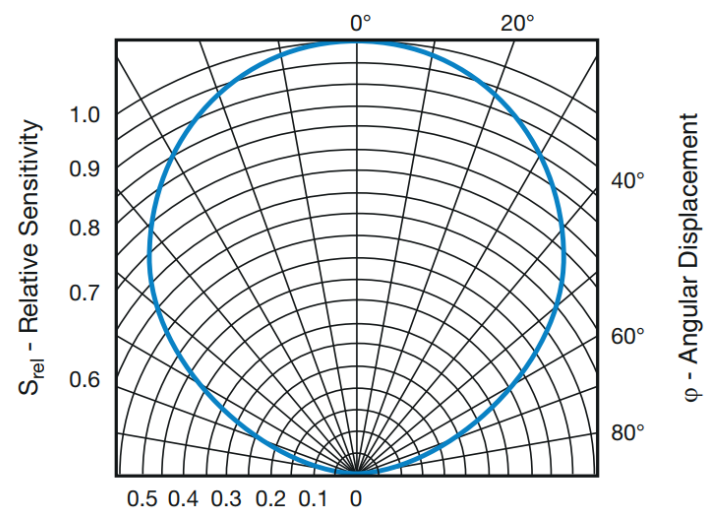

Figure 39 - Relative radiant sensitivity vs. angular displacement of the proximity sensor (reproduced from [57]) 


\section{References}

[1] Tekscan, "Pressure Mapping, Force Measurement \& Tactile Sensors," 2018. [Online]. Available: https://www.tekscan.com/.

[2] "Pressure Profile Systems." [Online]. Available: https://pressureprofile.com. [Accessed: 01-Feb-2018].

[3] M. Taylor, T. Grant, F. Knoefel, and R. Goubran, "Bed occupancy measurements using under mattress pressure sensors for long term monitoring of community-dwelling older adults," in MeMeA 2013 - IEEE International Symposium on Medical Measurements and Applications, Proceedings, 2013.

[4] B. R. Behrendt, A. M. Ghaznavi, M. Mahan, S. Craft, and A. Siddiqui, "Continuous bedside pressure mapping and rates of hospital- associated pressure ulcers in a medical intensive care unit.," vol. 23, no. 2, pp. 127-133, 2014.

[5] M. Cohen-McFarlane, J. Green, R. Goubran, and F. Knoefel, "Characterization of measurements from pressure sensitive mats using an anthropomorphic body model," 2016 IEEE Int. Instrum. Meas. Technol. Conf. Proc., pp. 1-4, 2016.

[6] M. Cohen-McFarlane, S. Member, J. R. Green, S. Member, R. Goubran, and I. Fellow, "Smart Monitoring of Fluid Intake and Bladder Voiding using Pressure Sensitive Mats," pp. 4921-4924, 2016.

[7] G. Hellard and R. a Russell, "A Robust, Sensitive and Economical Tactile Sensor for a Robotic Manipulator," Australas. Conf. Robot. Autom., no. November, pp. 1-5, 2002.

[8] G. De Maria, C. Natale, and S. Pirozzi, "Force/tactile sensor for robotic applications," 
Sensors Actuators, A Phys., vol. 175, pp. 60-72, 2012.

[9] Y. Ohmura, Y. Kuniyoshi, and A. Nagakubo, "Conformable and scalable tactile sensor skin for a curved surfaces," Proc. - IEEE Int. Conf. Robot. Autom., vol. 2006, no. May, pp. 13481353, 2006.

[10] Y. L. Park, S. C. Ryu, R. J. Black, K. K. Chau, B. Moslehi, and M. R. Cutkosky, "Exoskeletal force-sensing end-effectors with embedded optical fiber-bragg-grating sensors," IEEE Trans. Robot., vol. 25, no. 6, pp. 1319-1331, 2009.

[11] A. Cirillo, P. Cirillo, G. De Maria, C. Natale, and S. Pirozzi, "An artificial skin based on optoelectronic technology," Sensors Actuators, A Phys., vol. 212, pp. 110-122, 2014.

[12] “2010 Canada Year Book - Population and demography,” Stat. Canada, pp. 313-326, 2010.

[13] D. Peretz, A. Arnaert, and N. N. Ponzoni, "Determining the cost of implementing and operating a remote patient monitoring programme for the elderly with chronic conditions: A systematic review of economic evaluations," J. Telemed. Telecare, vol. 24, no. 1, pp. 1321, 2018.

[14] B. Wallace, T. El Harake, R. Goubran, N. Valech, and F. Knoefel, "Preliminary Results for Measurement and Classification of Overnight Wandering by Dementia Patient using MultiSensors," 2018 IEEE Int. Instrum. Meas. Technol. Conf., pp. 1-6, 2018.

[15] Seniors' falls in Canada. Ottawa: Public Health Agency of Canada, 2014.

[16] G. R. Yavuz et al., "A Smartphone Based Fall Detector with Online Location Support," Int. Work. Sens. App Phones, no. January 2010, pp. 31-35, 2010. 
[17] S. Abbate, M. Avvenuti, F. Bonatesta, G. Cola, P. Corsini, and A. Vecchio, "A smartphonebased fall detection system," Pervasive Mob. Comput., vol. 8, no. 6, pp. 883-899, 2012.

[18] M. Aslan, A. Sengur, Y. Xiao, H. Wang, M. C. Ince, and X. Ma, "Shape feature encoding via Fisher Vector for efficient fall detection in depth-videos," Appl. Soft Comput. J., vol. 37, pp. 1023-1028, 2015.

[19] S. G. Miaou, P. H. Sung, and C. Y. Huang, "A customized human fall detection system using omni-camera images and personal information," Conf. Proc. - 1st Transdiscipl. Conf. Distrib. Diagnosis Home Heal. D2H2 2006, vol. 2006, pp. 39-42, 2006.

[20] B. Mirmahboub, S. Samavi, N. Karimi, and S. Shirani, "Automatic monocular system for human fall detection based on variations in silhouette area," IEEE Trans. Biomed. Eng., vol. 60, no. 2, pp. 427-436, 2013.

[21] Y. Fan, M. D. Levine, G. Wen, and S. Qiu, “A deep neural network for real-time detection of falling humans in naturally occurring scenes," Neurocomputing, vol. 260, pp. 43-58, 2017.

[22] M. Salman Khan, M. Yu, P. Feng, L. Wang, and J. Chambers, “An unsupervised acoustic fall detection system using source separation for sound interference suppression," Signal Processing, vol. 110, pp. 199-210, 2015.

[23] Y. Zigel, D. Litvak, and I. Gannot, "A method for automatic fall detection of elderly people using floor vibrations and soundProof of concept on human mimicking doll falls," IEEE Trans. Biomed. Eng., vol. 56, no. 12, pp. 2858-2867, 2009.

[24] C. Garripoli et al., "Embedded DSP-based telehealth radar system for remote in-door fall 
detection," IEEE J. Biomed. Heal. Informatics, vol. 19, no. 1, pp. 92-101, 2015.

[25] B. Y. Su, K. C. Ho, M. J. Rantz, and M. Skubic, "Doppler radar fall activity detection using the wavelet transform," IEEE Trans Biomed Eng, vol. 62, no. 3, pp. 865-875, 2015.

[26] F. Bianchi, S. J. Redmond, M. R. Narayanan, S. Cerutti, and N. H. Lovell, "Barometric pressure and triaxial accelerometry-based falls event detection," IEEE Trans. Neural Syst. Rehabil. Eng., vol. 18, no. 6, pp. 619-627, 2010.

[27] K. Chaccour, R. Darazi, A. Hajjam El Hassans, and E. Andres, "Smart carpet using differential piezoresistive pressure sensors for elderly fall detection," 2015 IEEE 11th Int. Conf. Wirel. Mob. Comput. Netw. Commun. WiMob 2015, pp. 225-229, 2015.

[28] G. Feng, J. Mai, Z. Ban, X. Guo, and G. Wang, "Floor Pressure Imaging for Fall Detection with Fiber-Optic Sensors," IEEE Pervasive Comput., vol. 15, no. 2, pp. 40-47, 2016.

[29] A. Arshad, S. Khan, A. H. M. Z. Alam, A. F. Ismail, and R. Tasnim, "Capacitive proximity floor sensing system for elderly tracking and fall detection," no. November, pp. $28-30$, 2017.

[30] M. Cohen-Mcfarlane, "Analysis of Contact Pressure for Monitoring Body Fluid Distribution," Carleton University, 2017.

[31] M. Cohen-McFarlane, J. Green, R. Goubran, and F. Knoefel, "Monitoring congestive heart failure using pressure-sensitive mats," in 2016 IEEE International Symposium on Medical Measurements and Applications, MeMeA 2016 - Proceedings, 2016, pp. 1-6.

[32] N. Tsuchiya, K. Yamamoto, H. Nakajima, and Y. Hata, "A comparative study of heart rate estimation via air pressure sensor," 2008 IEEE Int. Conf. Syst. Man Cybern., pp. 3077- 
3082, 2008.

[33] D. C. Mack, J. T. Patrie, P. M. Suratt, R. A. Felder, and M. Alwan, "Development and preliminary validation of heart rate and breathing rate detection using a passive, ballistocardiography-based sleep monitoring system," IEEE Trans. Inf. Technol. Biomed., vol. 13, no. 1, pp. 111-120, 2009.

[34] G. G. Mora, J. M. Kortelainen, E. R. P. Hernández, M. Tenhunen, A. M. Bianchi, and M. O. Méndez, "Evaluation of Pressure Bed Sensor for Automatic SAHS Screening," IEEE Trans. Instrum. Meas., vol. 64, no. 7, pp. 1935-1943, 2015.

[35] S. S. Gilakjani, H. Azimi, M. Bouchard, R. A. Goubran, and F. Knoefel, "Improved sensor selection method during movement for breathing rate estimation with unobtrusive pressure sensor arrays," in 2018 IEEE Sensors Applications Symposium (SAS), 2018, pp. 1-6.

[36] M. H. Jones, R. Goubran, and F. Knoefel, "Reliable respiratory rate estimation from a bed pressure array," Annu. Int. Conf. IEEE Eng. Med. Biol. - Proc., pp. 6410-6413, 2006.

[37] Z. T. Beattie, C. C. Hagen, M. Pavel, and T. L. Hayes, "Classification of breathing events using load cells under the bed," Proc. 31st Annu. Int. Conf. IEEE Eng. Med. Biol. Soc. Eng. Futur. Biomed. EMBC 2009, pp. 3921-3924, 2009.

[38] D. Townsend, R. Goubran, F. Knoefel, and J. Leech, "Validation of unobtrusive pressure sensor array for central sleep apnea screening," IEEE Trans. Instrum. Meas., 2012.

[39] M. Holtzman, R. Goubran, F. Knoefel, and J. Pereira, "Measurement of respiratory changes in palliative care," 2012 IEEE I2MTC - Int. Instrum. Meas. Technol. Conf. Proc., pp. 14721476, 2012. 
[40] M. Taylor, R. Goubran, and F. Knoefel, "Patient standing stability measurements using pressure sensitive floor sensors," 2012 IEEE I2MTC - Int. Instrum. Meas. Technol. Conf. Proc., pp. 1275-1279, 2012.

[41] M. Taylor et al., "Assessing standing stability of older adults using pressure sensitive arrays," MeMeA 2012 - 2012 IEEE Symp. Med. Meas. Appl. Proc., pp. 10-14, 2012.

[42] "Tekscan - Pressure Mapping, Force Measurement \& Tactile Sensors," 2018. [Online]. Available: https://www.tekscan.com/body-pressure-mapping.

[43] S. Nizami, M. Cohen-mcfarlane, J. R. Green, and R. Goubran, "Comparing metrological properties of pressure-sensitive mats for continuous patient monitoring," pp. 1-6, 2017.

[44] "The difference between: Force Measurement Techniques," Machine Design. [Online]. Available: https://www.tekscan.com/resources/articles-research/difference-between-forcemeasurement-techniques. [Accessed: 01-Dec-2018].

[45] "Force Sensors in Robotic Design - Low-Profile, Low-Power, High-Performing Tactile Sensors." [Online]. Available: https://www.tekscan.com/resources/ebook/force-sensors-inrobotic-design. [Accessed: 01-Dec-2018].

[46] Á. Pintér and A. Huba, "Study of Pressure-Sensitive Materials for Floor Sensor Networks," Period. Polytech. Mech. Eng., vol. 60, no. 1, pp. 32-40, 2016.

[47] H. Böse, E. Fuß, and J. Ehrlich, "Capacitive Sensor Mats for Pressure Detection with High Sensitivity," AMA Conf. 2015, pp. 55-60, 2015.

[48] S. J. Woo, J. H. Kong, D. G. Kim, and J. M. Kim, “A thin all-elastomeric capacitive pressure sensor array based on micro-contact printed elastic conductors," J. Mater. Chem. C, vol. 2, 
no. 22, pp. 4415-4422, 2014.

[49] A. G. Mignani and F. Baldini, "Biomedical sensors using optical fibres," Reports Prog. Phys., vol. 59, no. 1, pp. 1-28, 1996.

[50] C.-A. Bunge and H. Poisel, "Sensors Based on Polymer Optical Fibers Microstructured and Solid Fibers," in Optical, Acoustic, Magnetic, and Mechanical Sensor Technologies, 2012, p. 357 .

[51] M. Donati et al., "A flexible sensor technology for the distributed measurement of interaction pressure," Sensors (Switzerland), vol. 13, no. 1, pp. 1021-1045, 2013.

[52] M. Donati, F. Cecchi, F. Bonaccorso, M. Branciforte, P. Dario, and N. Vitiello, "A modular sensorized mat for monitoring infant posture," Sensors (Switzerland), vol. 14, no. 1, pp. 510-531, 2013.

[53] S. G. Rabinovich, Measurement errors and uncertainties: Theory and Practice (3rd). 2005.

[54] C. M. Ferguson-Pell M. D., "Prototype Development and Comparative Evaluation of Wheelchair Pressure Mapping System,” Assist. Technol., vol. 5, no. 2, pp. 78-91, 1993.

[55] S. Soleimani Gilakjani, S. Bennett, R. A. Goubran, H. Azimi, M. Bouchard, and F. Knoefel, "Movement detection with adaptive window length for unobtrusive bed-based pressuresensor array," 2017 IEEE Int. Symp. Med. Meas. Appl. MeMeA 2017 - Proc., pp. 355-360, 2017.

[56] S. S. Gilakjani, H. Azimi, M. Bouchard, R. A. Goubran, and F. Knoefel, "Improved sensor selection method during movement for breathing rate estimation with unobtrusive pressure sensor arrays," in 2018 IEEE Sensors Applications Symposium (SAS), 2018, pp. 1-6. 
[57] Vishay, "Fully Integrated Proximity and Ambient Light Sensor with Infrared Emitter, I2C Interface, and Interrupt Function," 2014. 